\title{
Using Personality Measures for Selection Decisions: Predictive Utility and Applicants' Faking Behaviour
}

\author{
Georg Krammer
}

\begin{abstract}
Dissertation
submitted for the doctoral degree of natural sciences (Dr.rer.nat.) at the faculty of Natural Sciences of the University of Graz
\end{abstract}

Supervisor \& $1^{\text {st }}$ Assessor:

Univ.-Prof. Mag. Dr. Martin Arendasy

Psychological Methodology and Diagnostic, Department of Psychology,

University of Graz

$2^{\text {nd }}$ Assessor:

Ao.Univ.-Prof. Dr. Norbert Tanzer

Department of Psychology, University of Graz 


\section{Author's Declaration}

This dissertation is an independent and original academic work prepared and written entirely by myself. Thoughts and ideas that were taken from other sources are indicated as such and cited appropriately. The three publications this publication-based dissertation is based on fulfill the necessary requirements for publication-based dissertations.

Georg Krammer 


\section{Acknowledgments}

Just like a dissertation, everything in life is a process. Life is movement. It does not stand still. It grows, it flows. The experiences we have chosen for us mold us, form us. This process changes us. It does not occur by itself, it occurs if we let it transform us, and - more importantly - if we play an active part in it. That holds for every review, refusal, and acceptance I have ever gotten; on the small scale in publishing and science, and on the large scale in everything else.

For my dissertation, obviously I want to thank everyone who has accompanied me along the process of my dissertation. Thank you Martin for introducing me to measurement theory and sparking my interest. Thank you Markus for being the best co-author one could imagine. Thank you Barbara, for being my colleague, and becoming my friend; I am looking forward to all the times together that are to come. Thank you Regina for looking after me; I promise I will return the favour.

Most importantly, I want to thank all and everyone who have accompanied me alongside $m y$ process over the past years. Thank you Helga for always believing in me, and cherishing my honesty. Thank you Tobi, Mikey, Eva, Markus, Bernd, Gerhard, Birgit, Markus, Thomas, and Steffi for being the friends you are and for giving all the support you have given. In short, thank you for all my friends, who have become family.

Yes, life is a process. On we go... 


\begin{abstract}
Aim of this publication-based dissertation was to scrutinize the use of personality measures for selection decisions. This was done in three studies examining personality measures used for selection decisions to teacher education. First, Study 1 evaluated the predictive utility of personality measures for differentiated facets of academic achievement. In accordance with a large body of research, personality measures retained their predictive utility over and above other determinants of academic achievement. With Study 1 supporting the usefulness of personality measures for predicting future academic achievement in the academic field examined, Study 2 then addressed the prevalence of applicants' faking behaviour on the personality scales used for selection decisions. Study 2 showed that applicants increased their raw mean test scores in nearly all personality scales examined, and that applicants' faking behaviour could detrimentally affect applicants' rank order. Based on the findings of Study 2, Study 3 aimed to shed light on the effects of applicants' faking behaviour on the psychometric properties of the personality scales. Measurement invariance analyses revealed that applicants primarily faked by increasing their responses to selected items. The more heterogeneously the responses were increased, the more measurement properties were detrimentally affected. As the synopsis of the three studies, theoretical and practical implications for the use of personality measures for selection decisions are discussed.
\end{abstract}




\section{Zusammenfassung}

Das Ziel dieser publikationsbasierten Dissertation war es die Verwendung von Persönlichkeitsinventaren zu Selektionszwecken kritisch zu hinterfragen. Dafür wurden drei Studien zu der Verwendung von Persönlichkeitsinventaren zur Zulassung zu Lehramtsstudien durchgeführt. Studie 1 thematisierte die prädiktive Validität von Persönlichkeitsinventaren für differenzierte Facetten von Studienerfolg. Die prädiktive Validität von Persönlichkeitsmerkmalen für Studienerfolg konnte im Einklang mit bisheriger Forschung über andere Merkmale hinaus gezeigt werden. Aufbauend auf der prädiktiven Validität von Persönlichkeitsmerkmalen für Lehramtsstudienerfolg, befasste Studie 2 sich mit der Prävalenz des Faking-Verhaltens von BewerberInnen bei Persönlichkeitsskalen. Studie 2 zeigte auf, dass BewerberInnen ihre mittleren Testwerte in fast allen untersuchten Skalen erhöhten, und dass durch dieses Faking-Verhalten die Rangreihen der BewerberInnen verzerrt werden können. Studie 3 vertiefte diese Befunde, indem die psychometrischen Konsequenzen, die durch das Faking-Verhalten von BewerberInnen entstehen können, untersucht wurden. Die Messinvarianzanalysen zeigten, dass BewerberInnen hauptsächlich Faking-Verhalten zeigten indem sie ihre Antworten auf ausgewählten Items erhöhten; je heterogener diese Erhöhungen waren, umso mehr wurden Messeigenschaften beeinflusst. Abschließend werden die theoretischen und praktischen Implikationen der drei Studien diskutiert. 


\section{Contents}

$\begin{array}{lll}1 & \text { Introduction } & 1\end{array}$

2 Study 1: Predicting Academic Achievement in Teacher Education

2.1 Method . . . . . . . . . . . . . . . . . . . . . . 4

2.2 Results \& Discussion . . . . . . . . . . . . . . . . . . 5

3 Study 2: Do Applicants Fake? $\quad 7$

3.1 Method . . . . . . . . . . . . . . . 7

3.2 Results \& Discussion . . . . . . . . . . . . . . . . . . . . . . . 9

4 Study 3: Psychometric Effects of Applicants' Faking Behaviour 10

4.1 Method . . . . . . . . . . . . . . . . . . . . . . . . . . . 12

4.2 Results . . . . . . . . . . . . . . . . . . . . . . . 14

4.3 Additional Analysis: Adequacy of Sample Size . . . . . . . . . 15

4.4 Additional Analysis: Use of Fit-Indices . . . . . . . . . . . . . 15

4.5 Discussion . . . . . . . . . . . . . . . . . . . 25

$\begin{array}{llr}5 & \text { General Discussion } & 29\end{array}$

6 Full Texts \& Dissemination 32

6.1 Study 1: Realistic Job Expectations Predict Academic Achieve-

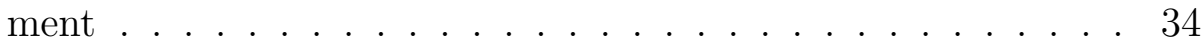

6.2 Study 2: Faking of Personality Measures for College Admission in Teacher Education . . . . . . . . . . . . . . . . 43

6.3 Study 3: The Psychometric Costs of Applicants' Faking: Examining Measurement Invariance and Re-test Correlations Across Response Conditions . . . . . . . . . . . . . . . . . . 54

\begin{tabular}{lll}
\hline 7 & References & 88
\end{tabular} 


\section{Introduction}

Just like selection processes in general, college admission processes are faced with faking (cf. Kuncel \& Hezlett, 2010) - a type of response bias wherein respondents distort their responses to self-descriptive items to be viewed more favourably (McFarland \& Ryan, 2000). The high stakes involved in selection decisions are likely to prompt some sort of faking behaviour from at least some applicants (cf. Dilchert, Ones, Viswesvaran, \& Deller, 2006; Tett \& Simonet, 2011). Faking may compromise the fairness of selection decisions, as applicants differ in their propensity and intensity to fake (e.g. Birkeland, Manson, Kisamore, Brannick, \& Smith, 2006; Mueller-Hanson, Heggestad, \& Thornton, 2003; Rosse, Stecher, Miller, \& Levin, 1998; Winkelspecht, Lewis, \& Thomas, 2006; Zickar \& Robie, 1999). Furthermore, due to faking, individual differences in test scores may not reflect only individual differences in the underlying trait, but also e.g. individual differences in the perceived relevance of items (cf. König, Merz, \& Trauffer, 2012; Ziegler, 2011). In line with this reasoning, previous studies indicate that faking can distort applicants' rank-order and thus subsequent selection decisions (e.g. Mueller-Hanson et al., 2003; Rosse et al., 1998; Winkelspecht et al., 2006).

Applicants' faking behaviour begins to pose a challenge for selection decisions as soon as measures are used where faking is possible. Faking could be simply avoided by omitting self-descriptive measures and relying on cognitive measures. For example, general mental ability has proven its predictive utility for academic achievement (e.g. Hell, Trapmann, \& Schuler, 2007; Kuncel, Hezlett, Sarah, \& Ones, 2001; Kuncel, Hezlett, \& Ones, 2004; Kuncel, Wee, Serafin, \& Hezlett, 2010). However, personality traits have also proven their predictive utility for academic achievement (e.g. Ackerman \& Heggestad, 1997; Farsides \& Woodfield, 2003; O'Conner \& Paunonen, 2007; Paunonen \& Ashton, 2001; Poropat, 2009; Richardson, Abraham, \& Bond, 2012). While the predictive utility of personality traits is smaller than that of general mental ability, it prevails even when accounting for general mental ability (e.g. Farsides \& Woodfield, 2003; Poropat, 2009). Therefore, it would not seem wise to rely only on cognitive determinants of academic achievement for selection decisions. Consequently, applicants' faking behaviour needs to be addressed.

While the predictive utilities of cognitive and non-cognitive determinants of academic achievement seem well established, it is still necessary to consider their usefulness for specific academic fields. Even for general mental ability, studies can offer mixed results, as some meta-analyses argue for (e.g. Hell et al., 2007) and some against (e.g. Kuncel et al., 2001) its predictive utility for academic achievement being moderated by the academic field. The liter- 
ature can also offer an ambiguous view on non-cognitive determinants. For example emotional stability has often been hypothesized to predict academic achievement (cf. Ackerman \& Heggestad, 1997; Farsides \& Woodfield, 2003), has failed to do so for academic achievement irrespectively of the academic field ( $\rho=.02$ : Poropat, 2009), while retaining its predictive utility in specific academic fields (e.g. teacher education: Mayr, 2011).

These mixed results for cognitive and non-cognitive determinants of academic achievement may be attributable to tertiary education having elements unique to the respective academic field. Teacher education, for example, is of a highly applied nature world wide due to student-teaching (DarlingHammond \& Lieberman, 2012). For the performance in student-teaching, studies have failed to show the predictive utility of general mental ability ( $\rho=.04$ : Kuncel et al. 2004). However, student-teaching is often experienced as the most influential part of teacher education (Ronfeldt, 2012), and meta-analyses have shown its predictive utility for future teacher performance (e.g. D'Agostino \& Powers, 2009). Emotional stability, on the other hand, has shown its predictive utility for the teacher education and profession (cf. Mayr, 2011). The predictive utility of emotional stability for academic achievement in teacher education may also be attributable to the applied nature of this education, as thus the risks of the profession can already be risks during the education (Gold \& Roth, 1993). Therefore, the specific academic discipline should be considered, and even the use of well established cognitive and non-cognitive determinants for selection decisions should be tailored to the specific academic discipline.

Following this line of reasoning leads to three basic questions which need to be addressed for college admission processes in specific academic disciplines:

1. Should well established determinants of academic achievement be used, such as general mental ability and personality traits?

2. If personality traits are used, do applicants fake?

3. If applicants fake, does applicants' faking behaviour detrimentally affect the psychometric properties of the personality measures?

The aim of this publication-based dissertation is to address these three questions for college admission processes in teacher education. To do so, three studies were conducted and published, which subsequently address these three questions. In the following chapters, these three studies will be outlined and the main conclusions drawn. Where appropriate, additional analyses will be presented. For further details, please consult the full texts in Section 6.1. Section 6.2, and Section 6.3, respectively. 


\section{Study 1: Predicting Academic Achievement in Teacher Education}

The aim of Study 1 (cf. Section 6.1: Krammer, Sommer, \& Arendasy, 2016) was to examine the predictive utilities of determinants for academic achievement in teacher education. This was done by studying one cohort of students $(n=176)$ over their entire three year long bachelor's degree of education. Cognitive and non-cognitive determinants of academic achievement in teacher education were considered as predictors. For the academic achievement, all grades of the three year long bachelor's degree were used.

First off, defining and measuring academic achievement in teacher education was revised. Grade point averages (GPA) are often considered the golden standard for measuring academic achievement. Thus, most metaanalyses (e.g. Hell et al., 2007; Kuncel et al., 2001, 2004, Poropat, 2009; Richardson et al., 2012; Trapmann, Hell, Weigand, \& Schuler, 2007) resort to GPAs to measure academic achievement. However, GPAs can be differentiated according to different courses types, e.g. courses for general knowledge, domain-specific knowledge, or applied courses (cf. D'Agostino \& Powers, 2009, Gustafsson \& Balke, 1993). The usefulness of such a differentiation is supported by studies showing that GPAs obtained from different course types exhibit differential structural relations to determinants of academic achievement and occupational performance (cf. D'Agostino \& Powers, 2009: Gustafsson \& Balke, 1993; Reeve, 2004). Due to student-teaching, teacher education world wide includes applied courses (Darling-Hammond \& Lieberman, 2012). GPAs of these student-teaching courses can be seen as an instance of procedural knowledge (the course requirements are similar to future occupational performance: Kuncel et al., 2004). Therefore, it may prove useful to distinguish between courses conveying declarative knowledge and procedural knowledge in teacher education. Consequently, academic achievement in Study 1 was modeled by GPAs reflecting a differentiation between courses conveying declarative knowledge and procedural knowledge.

Three determinants of academic achievement were considered. The first determinant was general mental ability. General mental ability is one of the most important determinants of academic achievement (e.g. Credé \& Kuncel, 2008; Hell et al., 2007; Kuncel et al., 2001, 2004, 2010; Kuncel \& Hezlett, 2007). The second determinant was a personality trait, namely a facet of the Big Five domain factor emotional stability. Emotional stability has often been hypothesized to predict academic achievement (cf. Ackerman \& Heggestad, 1997; Farsides \& Woodfield, 2003). However, this domain factor has failing to do so $(\rho=.02$ : Poropat, 2009). Nevertheless, some of its facets are related to academic achievement (e.g. test anxiety: $-.21 \leq \rho \leq-.33$; re- 
actions to stress: $-.11 \leq \rho \leq-.14$; Ackerman \& Heggestad, 1997; Hembree, 1988: Richardson et al. 2012). Consequently, more specific facets of emotional stability may be useful for predicting academic achievement, such as reaction to stress (cf. Ackerman \& Heggestad, 1997). This may be especially important for the education to a profession, where 1) stress and burnout are prevalent (Schaarschmidt, 2005; Schaarschmidt \& Kieschke, 2007), 2) teachers' stress has been linked to important student behaviours, such as not paying attention in class $(r=.39)$ and hostility toward the teacher $(r=.40$ : Geving, 2007), and 3) the risks of the profession can already be risks during the education (Gold \& Roth, 1993). As a third and final determinant, realistic job expectations - the degree to which realistic expectations about prospective education and job are held - were introduced as a new potential predictor of academic achievement. Studies suggest that students may not always have realistic expectations of studying at university, and that providing them with realistic information on what to expect can reduces attrition (e.g. Braxton, Vesper, \& Hossler, 1995; Cooke, Sims, \& Peyrefitte, 1995; Earnest \& Dwyer, 2010). Providing such realistic information increases selfselection, organizational commitment, satisfaction, and possibly even academic achievement, by lowering the initial expectations (e.g. Braxton et al., 1995; Cooke et al., 1995; Earnest \& Dwyer, 2010). Unfortunately, studies so far have never examined individual differences in the expectations and their predictive utility for academic achievement.

The hypotheses for Study 1 were that the examined determinants of academic achievement are differentially linked to the different facets of academic achievement. The predictive utility of general mental ability should be higher for the declarative knowledge facet of academic achievement than for the procedural knowledge facet. In contrast, the predictive utility of the facet of emotional stability should be higher for the procedural knowledge facet than for the declarative knowledge facet. Finally, the predictive utility of realistic job expectations - the newly introduced potential predictor - was examined.

\section{$2.1 \quad$ Method}

Measures. Data for all predictors were collected within the college admission process of the University College of Teacher Education Styria. General mental ability was assessed by fluid intelligence with two subtests of the Intelligence Structure Battery (INSBAT: Arendasy et al., 2012): numericalinductive reasoning (NIR) and figural-inductive reasoning (FIR). The facet of emotional stability was assessed with three subscales forming the higher order factor health and recreational behaviour of the Inventory for Personality Assessment in Situations (IPS: Schaarschmidt \& Fischer, 2013). The 
realistic job expectations were assessed with a structured interview designed specifically for college admission in teacher education.

Sample. In the academic year 2011/12, 237 applicants were accepted and began to study at the University College of Teacher Education Styria. Of these 237 students, 176 finished their bachelor's degree three years later. These 176 graduates comprised the final sample.

Analyses. Structural equation models were used to evaluate the predictive utilities. The structural relations between the latent predictor and criterion variables were evaluated in a two-step process (cf. Mueller \& Hancock, 2008). First, the measurement models for the predictor and criterion variables were evaluated separately. Secondly, the two measurement models were combined into a full structural model to examine the predictive utilities of the latent predictors for the criteria. For parameter estimation a WLSMV estimator (cf. Lei, 2009; Savalei \& Rhemtulla, 2013) was used (lack of multivariate normality and some indicators constituted categorical variables). The structural equation models were calculated with lavaan (Rosseel, 2012).

\subsection{Results \& Discussion}

The measurement model of the predictor variables $\left(\chi^{2}[11]=17.655, p=\right.$ $.09, R M S E A<.01, C F I>.99, S R M R=.042)$ and the criterion variables $\left(\chi^{2}[8]=6.651, p=.575, R M S E A<.01, C F I>.99, S R M R=.029\right)$ fitted the data well. The final full structural model also fitted the data well $\left(\chi^{2}[71]=76.004, p=.319, R M S E A=.020, C F I=.980, S R M R=.054\right)$. The final full structural model with the standardized path coefficients, and explained variances of the criteria is depicted in Figure 1. As Figure1 shows, all predictors had predictive utility.

As hypothesized, the predictors were differentially linked to the different facets of academic achievement. On the one hand, these findings support that GPAs assessing academic achievement in teacher education should be differentiated between declarative and procedural knowledge facets. On the other hand, these findings suggest that selection decisions in college admission processes for teacher education would profit from not being solely based on cognitive determinants, such as general mental ability. Non-cognitive determinants, such as personality traits, can augment the selection decisions by considering facets of teacher education for which general mental ability fails to show predictive utility. Consequently, it needs to be addressed if applicants' fake on the personality measures to increase their chances of being admitted to their desired academic field of study. 


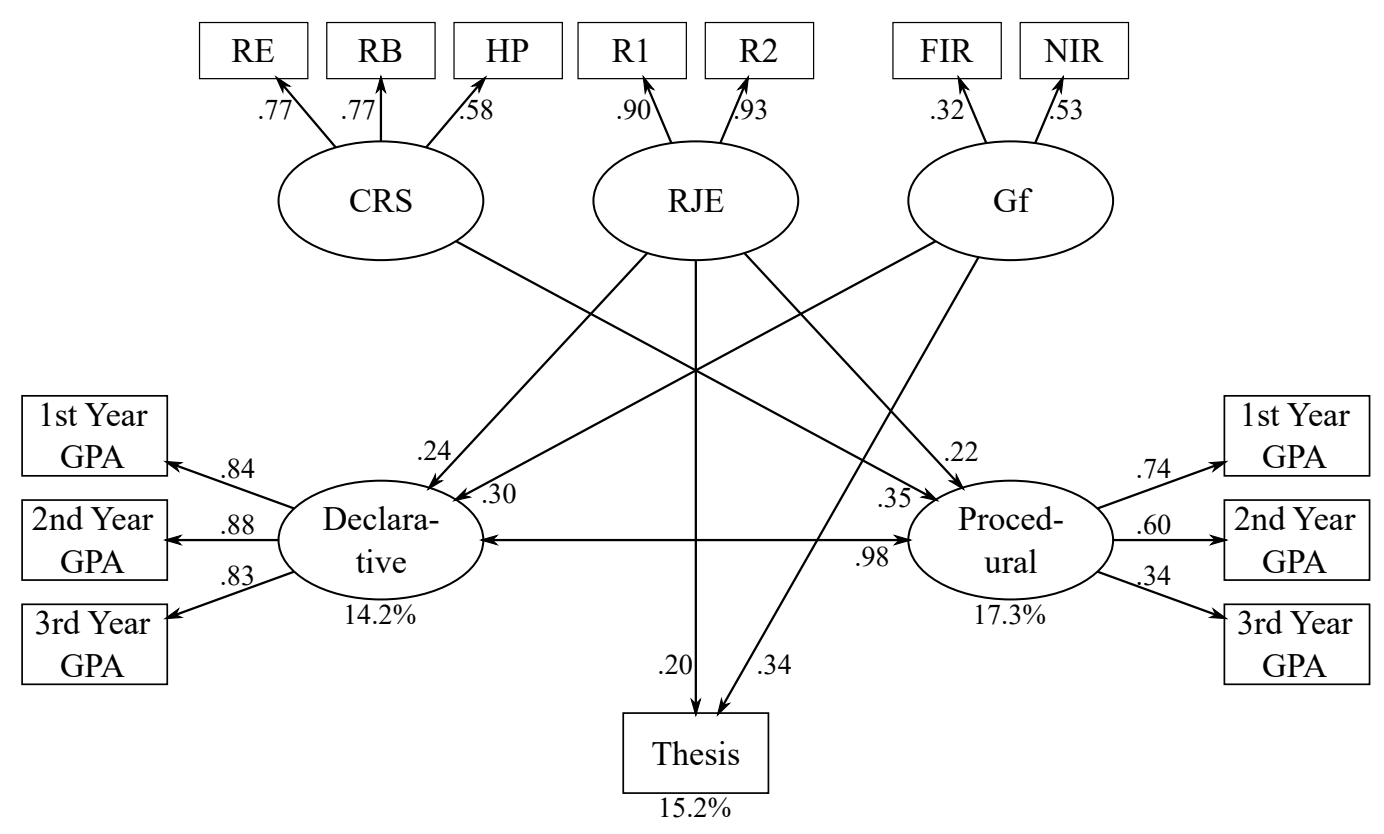

Figure 1: The final model $\left(\chi^{2}[71]=76.004, p=.319, R M S E A=.020\right.$, $C F I=.980, S R M R=.054)$ with standardized path coefficients. For the declarative knowledge, procedural knowledge, and the grade on the bachelor thesis, the amount of explained variance is given.

Note: CRS $=$ emotional stability facet; RJE $=$ realistic job expectations; Gf $=$ fluid intelligence; $\mathrm{RE}=$ relaxation after a working day; $\mathrm{RB}=$ active recovery behaviour in leisure; $\mathrm{HP}=$ health prevention because of warning signals; $\mathrm{R} 1$ and $\mathrm{R} 2=1^{\text {st }}$ and $2^{\text {nd }}$ Rater of the structured interview; NIR = numerical inductive reasoning; FIR = figural inductive reasoning, GPA $=$ grade point average. 


\section{Study 2: Do Applicants Fake?}

The aim of Study 2 (cf. Section 6.2, Krammer \& Pflanzl, 2015) was to assess if applicants to teacher education fake on the personality scales used in their college admission process. To this end, applicants were re-tested as incumbents $(n=116)$ half a year after they underwent their college admission process. The incumbents were re-tested with the same personality scales. Additionally, the incumbents were randomly assigned to three different response conditions. Aim of one of these response conditions was to obtain honest responses, to offer a comparison for the test scores obtained in the college admission setting.

For the practice of college admission processes, effects of applicants' faking behaviour on the selection decision are crucial. Scholars have argued that in real life selection settings effects of applicants' faking behaviour may be negligible (e.g. Bradley \& Hauenstein, 2006; Hogan, Barrett, \& Hogan, 2007; Ones, Dilchert, Viswesvaran, \& Judge, 2007). This view has been partially supported by a recent meta-analysis indicating only negligible to small differences between applicants' and incumbents' Big Five test scores $(0.11 \leq d \leq 0.45$ : Birkeland et al., 2006). It stands to reason, however, if this is applicable to the subsequent selection decisions. After all, selection settings offer the opportunity to distort one's own responses: applicants are motivated to make a good impression, personality measures are quite transparent, and there is no undisputed way to detect faking (Rosse et al., 1998). Moreover, in theoretical models of applicants' faking behaviour, applicants' faking is seen as an individual differences construct, always being at least partially determined by individual differences (e.g. Goffin \& Boyd, 2009; Marcus, 2009; McFarland \& Ryan, 2000, 2006; Mueller-Hanson, Heggestad, \& Thornton, 2006; Snell, Sydell, \& Lueke, 1999). Along this line of reasoning, faking behaviour has been shown to affect applicants' rank order, and thus subsequent selection decisions (e.g. Mueller-Hanson et al., 2003; Rosse et al., 1998; Winkelspecht et al., 2006).

Accordingly, it was hypothesized for Study 2 that applicants will distort their responses to present themselves more favourably than they normally would. Furthermore, there should be individual differences in this distortion of test scores, leading to a distortion of rank-order.

\subsection{Method}

Sample. In the respective academic year, 286 applicants were admitted to the University College of Teacher Education Styria. 121 thereof participated in Study 2 as incumbents. Of these 121 incumbents, it was possible 
$\mathrm{t} 1$

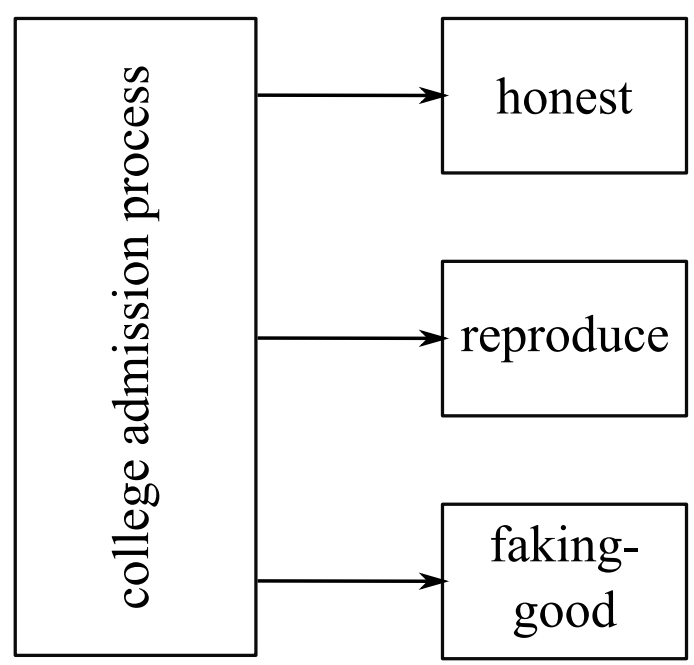

Figure 2: The combined within-subject and between-subject design of Study 2 and Study 3. At selection (t1), all respondents were applicants and went through the same college admission process. At re-test (t2), the same applicants were by now incumbents, and were randomly assigned to one of three response conditions.

to join the data for 116 with their college admission process. These 116 incumbents formed the final sample.

Design \& Procedure. All participants filled out the personality scales as part of the college admission process of the University College of Teacher Education Styria (selection, t1). Half a year later, they were asked to fill out the same personality scales a second time (re-test, t2). At re-test (t2), the participants were randomly assigned to one of three response conditions. The response conditions differed only in the instructions under which the personality scales were filled out. In the honest-condition, the standard instructions of the personality scales were used, to collect responses that were as honest as possible. In the other two conditions, participants were either asked to portrait themselves as favourably as possible to be admitted to their respective study major (faking-good-condition), or to try to respond to the personality scales like they did half a year ago when they were applicants (reproduce-condition). The complete $2 x 3$ design is depicted in Figure 2. The condition of interest for Study 2 is the honest-condition.

Measure. The personality inventory used was the Inventory for Personality Assessment in Situations (IPS: Schaarschmidt \& Fischer, 2013). The IPS is comprised of three higher-order factors: social and communicative be- 
haviour (6 subscales), health and recreational behaviour (6 subscales), and performance behaviour (3 subscales). Additionally, the IPS assesses the satisfaction with one's own behaviour in these three higher-order factors. All together, this yields 18 subscales. All 18 subscales were relevant for the selection decision.

Analyses. If applicants faked and if their rank-orders were affected was analysed by (M)ANOVAs and rank-order correlations, respectively. First, a MANOVA evaluated if there was an overall effect. Given an overall interaction between response condition and measurement point, ANOVAs evaluated the interaction for each subscale. If interactions were significant, post-test first examined the comparability of the response conditions at selection (t1), and then the differences within the response conditions between the measurement points. Finally, rank-order correlations examined the stability of the rank-order across selection (t1) and re-rest (t2). In the following, the focus will lie on the honest-condition.

\subsection{Results \& Discussion}

The MANOVA revealed an overall interaction between the response conditions and the measurement points $(F[36,194]=3.87, p<.01, \lambda=.26$, $\left.\eta^{2}=.42\right)$. The subsequent ANOVAs revealed interactions for all subscales (lowest effect: $F[2,113]=5.35, p<.01, \eta^{2}=.08$; largest effect: $F[2,113]=$ $59, p<.01, \eta^{2}=.51$ ). Comparing the mean test scores of selection (t1) and re-test ( $\mathrm{t} 2)$ in the honest-condition yielded effects between $d=-0.27$ and $d=-1.87$. These effects were significant for 16 out of 18 subscales $(-0.58 \leq d \leq-1.87)$. The rank-order correlations in the honest-condition were between $r=.17$ and $r=.70$. These rank-order correlations were significant for 14 out of 18 subscales.

The resulting distortion of mean responses showed that applicants increased their responses in the admission testing setting. For the subscales affected by applicants' faking behaviour, the range of the mean response distortion was higher than the range reported by a recent meta-analysis on applicants' faking behaviour in personal selection $(0.11 \leq d \leq 0.45$ : Birkeland et al. 2006). This may be attributable to the fact that the meta-analysis considered Big Five domain factor measures for all selection decisions. By contrast, the personality measure used for the selection decision to teacher education in Study 2 was a measure specifically fitted to the teaching profession (Schaarschmidt \& Fischer, 2013). For the latter, the content of the personality measure should therefore be attained as highly relevant, and this perceived relevance is appraised by applicants (e.g. Birkeland et al., 2006; Ziegler, 2011). 
The stabilities of rank-orders across applicants and incumbents further corroborated that applicants' faking behaviour should not be considered negligible. In general, the stability of the rank-orders was in line with previous studies, but had a larger range (cf. conscientiousness: $.50 \leq r \leq .62$; Big Five: $.46 \leq r \leq .68$; Griffith, Chmielowski, \& Yoshita, 2007; Hogan et al., 2007: Peterson, Griffith, Isaacson, O'Connell, \& Mangos, 2011). For some subscales, the stability of rank-order was given (e.g. $r=.7$ ). However, the rank-order was not preserved for 4 out of 18 subscales, and was still arguably low for some of the other 14 subscales. These results corroborate that applicants' faking behaviour can distort applicants' rank-order. This distortion points towards applicants' faking behaviour being influenced by individual differences of the applicants. Furthermore, the range of distortion across the subscales suggests that differences between the scales also influence applicants' faking behaviour (e.g. transparency of assessment: Marcus, 2009; McFarland \& Ryan, 2000, 2006).

In summary, if personality measures are used for selection decisions, applicants will show at least some amount of faking behaviour. To gauge how detrimental the effects of applicants' faking behaviour are, it is necessary to go beyond raw means and correlations, and examine the effects on the psychometric properties of the personality measures used.

\section{Study 3: Psychometric Effects of Applicants' Faking Behaviour}

The aim of Study 3 (cf. Section 6.3. Krammer, Sommer, \& Arendasy, in press) was to assess if and how applicants' faking behaviour affects the psychometric properties of the personality scales used for selection decisions. To do so, the measurement invariance (cf. Cheung \& Rensvold, 2002; Millsap, 2011; Mislevy et al., 2013; Raykov, Marcoulides, \& Li, 2012; Vandenberg \& Lance, 2000) across applicants' and incumbents was examined for three subscales of the IPS, one for each of the three higher-order factors of the IPS. Additional data ( $n=127 ; N=243$ ) was collected following the design of Study 2 (cf. Figure 2) to provide a sample size allowing the measurement invariance analyses. As in Study 2, the honest-condition will also be the focus of Study 3, i.e. the comparison between applicants' responses and their honest responses as incumbents half a year later.

Four levels of measurement invariance can be distinguished: 1) configural, 2) weak, 3) strong, and 4) strict measurement invariance. These levels of measurement invariance build upon each other, and they are tested level by level. Additionally, the invariance of latent variances, latent means, and latent covariances can be examined. 
Applicants' faking behaviour would have different practical implications, depending on the level of measurement invariance which is affected. If applicants' faking behaviour would affect configural measurement invariance, scores of incumbents and applicants could not be directly compared in a meaningful way (e.g. Cheung \& Rensvold, 2002; Millsap, 2011; Mislevy et al., 2013; Vandenberg \& Lance, 2000). If weak measurement invariance would be affected, then a nonuniform bias would be present (cf. Barendse, Oort, \& Garst, 2010, Penfield \& Camilli, 2007). Therefore, predictive validities derived from samples of incumbents would not apply to applicants Chen, 2008). If strong measurement invariance would be affected, a uniform bias would be present (cf. Barendse et al., 2010; Penfield \& Camilli, 2007). Consequently, mean scores differences between applicants and incumbents would not only reflect mean individual differences, but also measurement bias (cf. Chen, 2008; Cheung \& Rensvold, 2002; Li \& Zumbo, 2009; Millsap, 2011). Finally, if only strict measurement invariance would be affected, it could be argued that applicants' faking behaviour could have little practical implications.

Applicants' faking behaviour can theoretically affect measurement invariance on every level of measurement invariance. Consider a questionnaire for the Big Five domain factor extraversion and a teacher vacancy. The questionnaire may measure extraversion for incumbents but extraversion and faking intensity for applicants. Thus, configural measurement invariance would not be given across applicants and incumbents. Now, let on item of the questionnaire be "I like to stay in contact with parents". For applicants, this item content may be an indicator of their extraversion. Incumbents, by contrast, may view this item content as a necessary part of their job. In this example, the item would reflect individual differences in extraversion for applicants, but would do so less for incumbents. Consequently, weak measurement invariance would be affected. Now consider an applicant who would normally endorse "staying in contact with parents" only to a small degree. When applying for the teacher vacancy, however, the applicant may perceive this item as relevant for the teaching profession, and endorse this item to a large degree. Were this the case, strong measurement invariance would be affected. Finally, consider the need of applicants for the job. The individual differences in this need would be a potential source of systematic variance, thus introducing variance unaccounted for by the psychometric model. In this case, strict measurement invariance would be affected.

It was hypothesized for Study 3 that strict measurement invariance should hold across applicants and incumbents, with only moderate re-test correlations and higher latent means of the applicants compared to the incumbents. Strict measurement invariance was hypothesized due to some scholars (e.g. 
Bradley \& Hauenstein, 2006; Hogan et al., 2007; Ones et al., 2007) arguing that applicants' faking behaviour should have only negligibly effects on their personality test scores. This line of reasoning is corroborated by one of the very few studies examining measurement invariance across applicants and incumbents (Robie, Zickar, \& Schmit, 2001). Moderate re-test correlations were hypothesized because studies indicate a distortion of applicants' rank order (cf. Mueller-Hanson et al., 2003; Rosse et al., 1998; Winkelspecht et al., 2006), and only moderate re-test correlations were found between applicants and incumbents (e.g. Griffith et al., 2007; Griffin \& Wilson, 2012; Peterson et al., 2011). Finally, latent mean differences were hypothesized because applicants have shown higher mean scores than incumbents (Birkeland et al., 2006).

\subsection{Method}

Sample \& Procedure. The data was collected in the same way as in Study 2 (cf. Section 3.1). The final sample was 243 incumbents, for whom it was possible to join the data across selection (t1) and re-test (t2).

Measure. As in Study 1 and Study 2, the Inventory for Personality Assessment in Situations was used (IPS: Schaarschmidt \& Fischer, 2013). To study applicants' faking behaviour, one subscale of each of the three higherorder factors of the IPS was selected: activity in familiar communicative situation was chosen for the higher-order factor social and communicative behaviour, preventive health behaviour in response to warning signals for the higher-order factor health and recreational behaviour, and self-confidence in test situation for the higher-order factor performance behaviour.

Analyses. Multi-group means and covariance structure models were computed. Two measurement models formed the basis for the model, one for each measurement point. The residuals of the same items in the measurement models were allowed to correlate between selection (t1) and re-test (t2) to account for the re-administration. This model was specified for each response condition. The full model is depicted in Figure 3 .

Even though the respondents were randomly assigned to the three response conditions at re-test ( $\mathrm{t} 2$ ), in a first step, the comparability of the three response conditions at selection ( $\mathrm{t} 1$ ) was examined. Ideally, strict measurement invariance, and equal latent means and variances across the three response conditions should be given. Accordingly, constraints were imposed for weak $\left(\lambda_{t 1, h, 1}=\lambda_{t 1, r, 1}=\lambda_{t 1, f, 1}\right.$, etc. $)$, strong $\left(\tau_{t 1, h, 1}=\tau_{t 1, r, 1}=\tau_{t 1, f, 1}\right.$, etc. $)$, and strict $\left(\theta \epsilon_{t 1, h, 1}=\theta \epsilon_{t 1, r, 1}=\theta \epsilon_{t 1, f, 1}\right.$, etc. $)$ measurement invariance, followed by constraints for the latent trait variances $\left(\Phi_{t 1, h}=\Phi_{t 1, r}=\Phi_{t 1, f}\right)$ and means $\left(\xi_{t 1, h}=\xi_{t 1, r}=\xi_{t 1, f}\right)$. 
HONEST

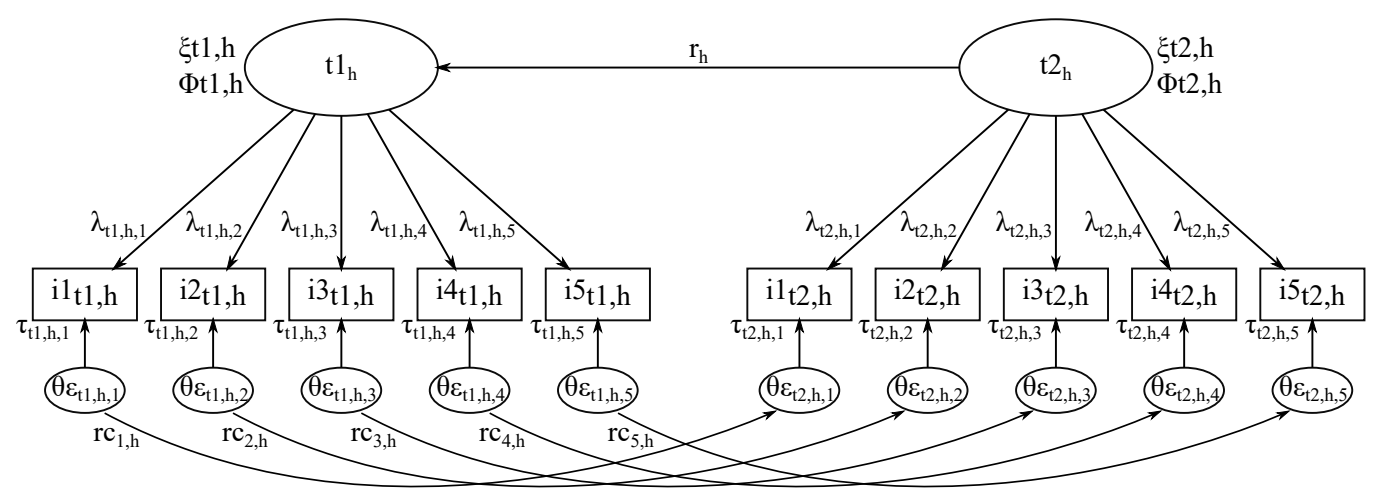

REPRODUCE

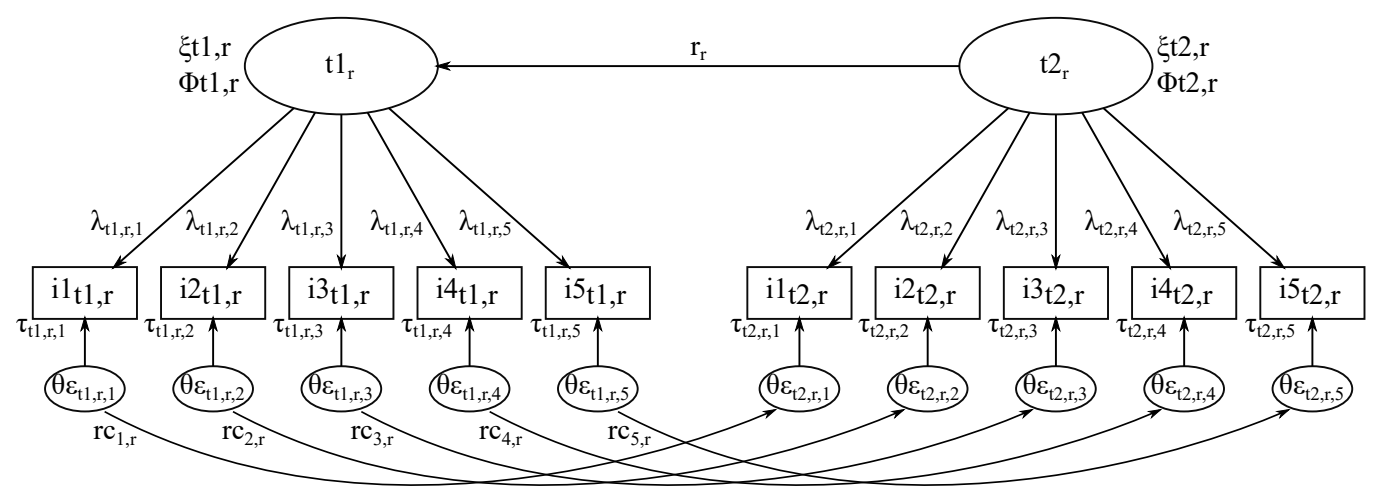

FAKING-GOOD

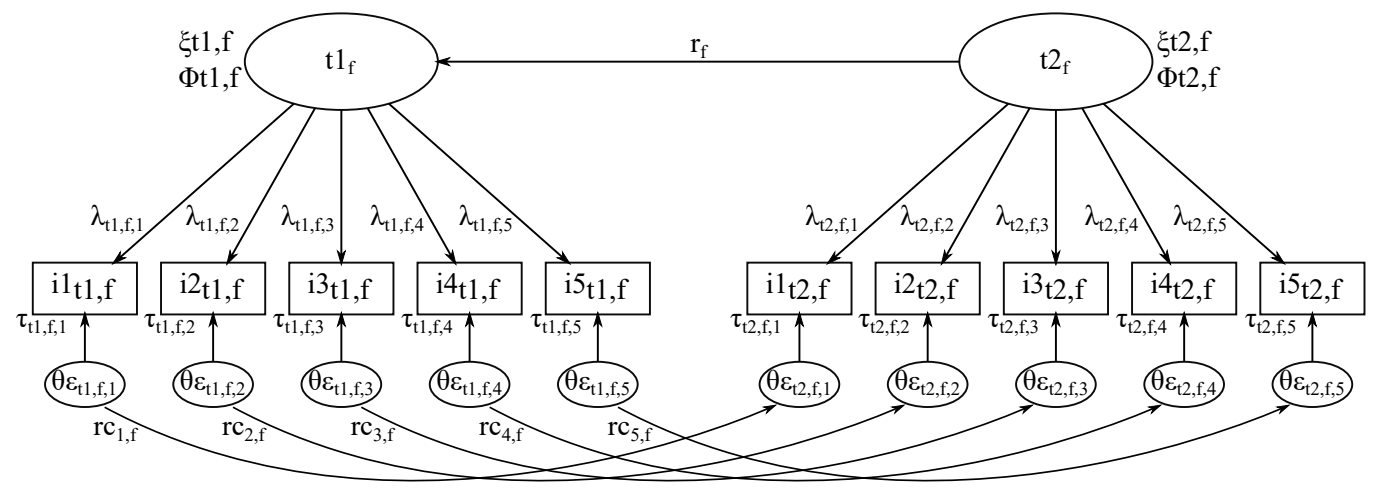

Figure 3: The multi-group means and covariance structure model, containing one-factor models at selection (t1) and at re-test (t2), and the covariances. The $\lambda \mathrm{s}$ denote the factor loadings, the $\tau \mathrm{s}$ the intercepts, the $\theta \epsilon \mathrm{s}$ the residual variances, the $r c$ s the residual covariances, the $\xi$ s the latent trait means, the $\Phi$ s the latent trait variances, and $r$ s the latent trait covariance. 
After examining the measurement invariance across the three response conditions at selection ( $\mathrm{t} 1$ ), the measurement invariance across the measurement points was examined. To this end, constraints were imposed to test for weak $\left(\lambda_{t 1, h, 1}=\lambda_{t 2, h, 1}, \lambda_{t 1, h, 2}=\lambda_{t 2, h, 2}\right.$, etc. $)$, strong $\left(\tau_{t 1, h, 1}=\tau_{t 2, h, 1}\right.$, $\tau_{t 1, h, 2}=\tau_{t 2, h, 2}$, etc. $)$, and strict $\left(\theta \epsilon_{t 1, h, 1}=\theta \epsilon_{t 2, h, 1}, \theta \epsilon_{t 1, h, 2}=\theta \epsilon_{t 2, h, 2}\right.$, etc. $)$ measurement invariance. Then, the residual covariances were constrained across the response conditions $\left(r c_{1, h}=r c_{1, r}=r c_{1, f}\right.$, etc.). Afterwards, constraints were imposed on the latent trait variances $\left(\Phi_{t 1, h}=\Phi_{t 2, h}\right.$, etc. $)$ and means $\left(\xi_{t 1, h}=\xi_{t 2, h}\right.$, etc. $)$.

\subsection{Results}

Comparability at Selection (t1). The unconstrained models fitted the data well for all three subscales (cf. upper parts of Tables 1-3 in Section 6.3). Imposing the step-by-step constraints across the three response conditions at selection (t1) did not worsen the model fits at any step $(0.083 \leq$ $p\left(\Delta \chi^{2}\right) \leq 0.702$; largest decrease in $\left.C F I=0.001997\right)$. Therefore, strict measurement invariance, equal latent trait variances, and equal latent trait means at selection ( $\mathrm{t} 1$ ) were given across the respondents randomly assigned to the three response conditions at re-test (t2) for all three scales.

Measurement Invariance Across the Measurement Points. Next, constraints were imposed to examine the measurement invariance across the measurement points (cf. lower parts of Tables 1-3 in Section 6.3, and Table 4 in Section 6.3). Weak measurement invariance across applicants and incumbents was given in the honest-condition for all three subscales. By contrast, strong measurement invariance was only given for the scale preventive health behaviour in response to warning signals, but not for the other two scales. All affected intercepts were higher at selection (t1) than at re-test (t2). In the next step, the residual variances were examined. The residual variances were equal for two scales, but not all were equal for preventive health behaviour in response to warning signals. In this scale, the two affected residual variances were lower at selection ( $\mathrm{t} 1$ ) than at re-test ( $\mathrm{t} 2)$.

Latent Variances, Means, and Covariances. For no subscale the latent variances and means were equal across applicants and incumbents in the honest-condition (cf. Tables 4 in Section 6.3). Either, the latent means were equal but the variances were not (activity in familiar communicative situation), or the latent variances were equal but the means were not (preventive health behaviour in response to warning signals), or both were not equal (self-confidence in test situation). If the latent means were affected, they were higher for applicants than for incumbents. If the latent variances were affected, they were lower for applicants than for incumbents. Lastly, 
Study 3: Psychometric Effects of Applicants' Faking Behaviour

the latent re-test correlations were $r=.497, r=.387$, and $r=.533$ for the three subscales, respectively.

\subsection{Additional Analysis: Adequacy of Sample Size}

The sample size in Study 3 was rather small per group for measurement invariance analyses. Due to this concern, subsequent Monte Carlo simulation studies were conducted. Monte Carlo simulation studies were carried out for each scale to test the stability of the parameters estimates and thereby the adequacy of the sample size for the analyses. The final models of each subscale were used as the population models for the Monte Carlo simulations, which were carried out with 500 repetitions each.

The Monte Carlo simulation studies verified the stability of the parameters estimates. Only for the scale activity in familiar communicative situation there were some small biases: there was a small bias in the SEs of the factor loadings of two items and in the latent means. In spite of the slight bias in the SEs of the factor loadings, the factor loadings were significant in $100 \%$ of the simulations, and the estimates themselves were unaffected. Furthermore, the latent means may have been also slightly affected, however latent means of the Monte Carlo simulation exhibited the same pattern as the latent means of the data. A detailed summary of the Monte Carlo simulation studies can be found in Table 1, Table 2, and Table 3 for the scale activity in familiar communicative situation, in Table 4, Table 5, and Table 6 for the scale preventive health behaviour in response to warning signals, and in Table 7. Table 8, and Table 9 for the scale self-confidence in test situation.

\subsection{Additional Analysis: Use of Fit-Indices}

In the current study, the widely used SRMR was not used to assess the model fit. Evaluating the SRMR in the final models showed values exceeding the usual cut-off of .08 (final models: .107, .141, and .141, respectively). However, studies have suggested that the SRMR tends to be higher with smaller sample sizes (e.g. Chen, 2008; Heene, Hilbert, Draxler, Ziegler, \& Bühner, 2011; Meade, Johnson, \& Braddy, 2008). Therefore, it would be desirable to assess whether or not the SRMR was detrimentally affected by the sample size before rejecting the models due to the SRMR.

To test the effect of sample size on the SRMR, a second Monte Carlo simulation study was conducted. This Monte Carlo simulation study again assumed the final models as population models. Simulations (500 repetitions each) were carried out with varying sample sizes (81, 120, 250, and 500 per group). For each drawn sample, the respective final model of the respective 
Study 3: Psychometric Effects of Applicants' Faking Behaviour

Table 1:

Comparison of the parameter estimates (and SEs) and the parameter estimates of the Monte Carlo simulation study (and SEs), the magnitude of the resulting absolute bias, and percentage of significant parameter estimates for the scale activity in familiar communicative situation in the honest-condition.

\begin{tabular}{|c|c|c|c|c|c|c|c|c|c|c|}
\hline & \multicolumn{4}{|c|}{ Parameter Estimate } & \multicolumn{5}{|c|}{ Parameter Estimate's SE } \\
\hline & & Estimate & M & $\mathrm{SD}$ & Bias & $\mathrm{SE}$ & M & SD & Bias & Power \\
\hline \multirow{10}{*}{ 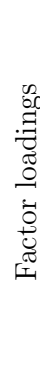 } & t1_i1 & 1 & - & - & - & - & - & - & - & - \\
\hline & t1_i2 & 5 & 19122 & 14010 & .00187 & 0.16116 & 0.13413 & 0.01895 & 0.02704 & $100.00 \%$ \\
\hline & t1_i3 & 1.18019 & 1.18951 & 0.12949 & -0.00931 & 0.12299 & 0.11746 & & 0.00553 & $100.00 \%$ \\
\hline & t1_i4 & & 1.14093 & 0.15460 & -0.01238 & 0.24416 & 0.14491 & 0.01900 & 0.09925 & $100.00 \%$ \\
\hline & t1_i5 & 1.093 & 1.09091 & 0.12734 & 0.00216 & 0.20547 & 0.12316 & 0.01685 & 0.08232 & $100.00 \%$ \\
\hline & t2_i1 & 1 & - & - & 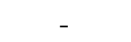 & 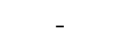 & 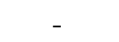 & 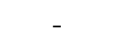 & & 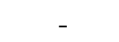 \\
\hline & t2_i2 & .18 & 1.19122 & 14316 & .00 & 0.16116 & 0.13413 & 0.018 & 0.02 & 100.0 \\
\hline & t2_i3 & & 1.18 & 12949 & -0.00931 & 0.12 & 0.11 & 0. & 0.0 & $100.00 \%$ \\
\hline & & & & & & & & & 0.0 & $100.00 \%$ \\
\hline & $\mathrm{t} 2 \_\mathrm{i} 5$ & & 10 & & & & 0.12316 & & & \\
\hline \multirow{10}{*}{ 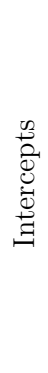 } & .1 & & 38120 & תמתפס & & 0.01615 & 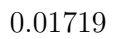 & & & \\
\hline & $\mathrm{t}] \mathrm{ra} \cdot \mathrm{cos}$ & & 3.2 & & & & & & & \\
\hline & t1_i3 & & 3.67 & & & & 0.0 & & -0.0 & $0 \%$ \\
\hline & $\mathrm{t} 1 \mathrm{i}$ & & 2.96 & & & & 0.0 & & 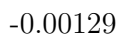 & $0 \%$ \\
\hline & t1_is & & 3.56 & & & & 0.0 & & -0 . & $0 \%$ \\
\hline & t2_i1 & & 3.6 & & & & & & & \\
\hline & t2_i2 & & 3.09 & & & & & & & \\
\hline & t2_i3 & & 3.49 & & & & & & & \\
\hline & $\mathrm{t}$ & & 2.9 & & & & & & & \\
\hline & $\mathrm{t} 2 \_\mathrm{i}$ & & 3.37 & & & & 0.0 & & -0 . & 100 \\
\hline \multirow{10}{*}{ 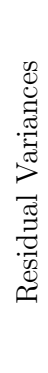 } & & & & & & & & & & \\
\hline & $\mathrm{t} 1 \_\mathrm{i}$ & & 0 & & & & & & & $0 \%$ \\
\hline & t1_i & & & & & & & & & $00 \%$ \\
\hline & $\mathrm{t} 1 \_\mathrm{i}$ & & & & & & & & & \\
\hline & t1_i & & 4 & & & & 0.1 & & & \\
\hline & t2_i1 & & 0 & & & & & & & $0 \%$ \\
\hline & t2_i2 & & & & & & & & & $0 \%$ \\
\hline & t2_i3 & & 0.15889 & & & & & & 0.0 & $100.00 \%$ \\
\hline & t2_i4 & & 0.36872 & & & & & & & $100.00 \%$ \\
\hline & $\mathrm{t} 2 \_\mathrm{i} 5$ & 0.2 & 0.23424 & 0.01759 & 0.00127 & 0.0 & 0.01743 & 0.0 & 0.0 & $100.00 \%$ \\
\hline \multirow{5}{*}{$\begin{array}{c}\overrightarrow{0} \\
\dot{0} \\
1 \\
\dot{d} \\
\dot{0}\end{array}$} & 11 & & & & & & & & & \\
\hline & i2 & & & & & & & & & \\
\hline & i3 & & & & & & & & & $0 \%$ \\
\hline & i4 & & 0.11549 & 0.02779 & 0.00034 & 0.02 & 0.02642 & 0.0 & -0.0 & $99.60 \%$ \\
\hline & i5 & 0.04074 & 0.04111 & 0.01666 & -0.00036 & 0.01 & 0.01683 & 0.00161 & 0.00002 & $69.20 \%$ \\
\hline \multirow{5}{*}{ 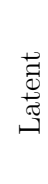 } & $\mathrm{t}$ & & & & & & & & & \\
\hline & $\mathrm{t} 2 \xi$ & & & & & & & & 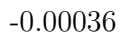 & $70.00 \%$ \\
\hline & t1 $\Phi$ & & & & & & & & & $100.00 \%$ \\
\hline & t2 $\Phi$ & & & & & & & & & $100.00 \%$ \\
\hline & $\mathrm{r}$ & 0.04651 & 0.04682 & 0.01381 & -0.00031 & 0.01619 & 0.01391 & 0.00245 & 0.00229 & $95.00 \%$ \\
\hline
\end{tabular}


Study 3: Psychometric Effects of Applicants' Faking Behaviour

Table 2:

Comparison of the parameter estimates (and SEs) and the parameter estimates of the Monte Carlo simulation study (and SEs), the magnitude of the resulting absolute bias, and percentage of significant parameter estimates for the scale activity in familiar communicative situation in the reproducecondition.

\begin{tabular}{|c|c|c|c|c|c|c|c|c|c|c|}
\hline & \multicolumn{4}{|c|}{ Parameter Estimate } & \multicolumn{5}{|c|}{ Parameter Estimate's SE } \\
\hline & & Estimate & M & $\mathrm{SD}$ & Bias & SE & M & SD & Bias & Power \\
\hline \multirow{10}{*}{ 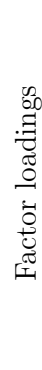 } & i1 & 1 & - & - & - & - & - & - & - & - \\
\hline & $\mathrm{t} 1$ in2 & 1.18935 & 1.19122 & 0.14316 & 00187 & .16116 & 0.13413 & 0.01895 & 0.02704 & $100.00 \%$ \\
\hline & $\mathrm{t} 1 \_\mathrm{i} 3$ & 1.18019 & 1.18951 & 0.12949 & -0.00931 & 0.12299 & 0.11746 & 0.01765 & 0.00553 & $100.00 \%$ \\
\hline & t1_i4 & 1.12855 & 1.14093 & 0.15460 & -0.01238 & 0.24416 & 0.14491 & 0.01900 & 0.09925 & $100.00 \%$ \\
\hline & $\mathrm{t} 1 \_\mathrm{i} 5$ & 1.09307 & 1.09091 & 0.12734 & 0.00216 & 0.20547 & 0.12316 & 0.01685 & 0.08232 & $100.00 \%$ \\
\hline & $\mathrm{t} 2 \_\mathrm{i} 1$ & 1 & - & - & - & - & - & 年 & & 年 \\
\hline & $\mathrm{t} 2 \_\mathrm{i} 2$ & 1.18935 & 1.19122 & 0.14316 & -0.00187 & 0.16116 & 0.13413 & 0.01895 & 0.02704 & $100.00 \%$ \\
\hline & $\mathrm{t} 2 \_\mathrm{i} 3$ & 1.18019 & 1.18951 & 0.12949 & -0.00931 & 0.12299 & 0.11746 & 0.01765 & 0.00553 & $100.00 \%$ \\
\hline & t2_i4 & 1.12855 & 1.14093 & 0.15460 & -0.01238 & 0.24416 & 0.14491 & 0.01900 & 0.09925 & $100.00 \%$ \\
\hline & $\mathrm{t} 2$ i 5 & 1.09307 & 1.09091 & 0.12734 & 0.00216 & 0.20547 & 0.12316 & 0.01685 & 0.08232 & $100.00 \%$ \\
\hline \multirow{10}{*}{ 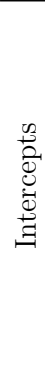 } & $\mathrm{t}$ & 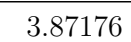 & 3.8 & 5 & 2 & 0.01 & 19 & 93 & 94 & $100.00 \%$ \\
\hline & $\mathrm{t}$ & & 3.2 & & & & & & & $0 \%$ \\
\hline & t1_i: & & 3.67 & & & & & & & \\
\hline & t1 i & & 2.9 & & & 0.02 & 0.03014 & & & $.00 \%$ \\
\hline & $\mathrm{t} 1 \mathrm{i} 5$ & & 3.5 & 0 . & & 0.02 & 0.0 & & & $100.00 \%$ \\
\hline & t2_i1 & & 3.81 & 0.03905 & 2 & 0.01 & 0.0 & 93 & -0. & $100.00 \%$ \\
\hline & $\mathrm{t} 2 \_\mathrm{i} 2$ & 3. & 3.29 & 0.05088 & 02 & 0.02 & 0.02 & 0.00156 & -0.0 & $100.00 \%$ \\
\hline & $\mathrm{t} 2$ i 3 & & 3.6 & 0.0 & 7 & 0.02 & & 0.0 & -0. & $100.00 \%$ \\
\hline & $\mathrm{t} 2 \_\mathrm{i} 4$ & & 2.96 & 0.05 & 7 & 0.02 & 0.0 & 97 & -0.0 & $100.00 \%$ \\
\hline & $\mathrm{t} 2 \_\mathrm{i} 5$ & 3.62861 & 3.56856 & 0.04740 & 0.06005 & 0.02361 & 0.02458 & 0.00145 & -0.00097 & $100.00 \%$ \\
\hline \multirow{10}{*}{ 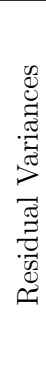 } & t. & 0.08980 & 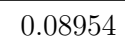 & 9 & 0.00026 & 0.01104 & 1 & 8 & 33 & $00 \%$ \\
\hline & $\mathrm{t} 1 \_\mathrm{i} 2$ & & 0.2 & 0.02223 & & 0.02 & & & & $100.00 \%$ \\
\hline & $\mathrm{t} 1$ i 3 & & 0.15889 & 0.01380 & 0.00071 & 0.01 s & & & & $100.00 \%$ \\
\hline & t1_i4 & & & 0.02863 & & 0.02 & & & & $100.00 \%$ \\
\hline & $\mathrm{t} 1$ it5 & & & & & & & & & $100.00 \%$ \\
\hline & t2_i1 & & 0.0 & 0.0 & & 001 & 0.0 & & 0.0 & $.00 \%$ \\
\hline & $\mathrm{t} 2$ id2 & & 0.2 & 3 & 6 & $002-2$ & 0.0 & 0.0 & 0.0 & $100.00 \%$ \\
\hline & $\mathrm{t} 2 \_\mathrm{i} 3$ & 0.15961 & 0.15 & 0.01 & 0.00071 & 0.01 & 0.01330 & 0.00125 & 0.00627 & $100.00 \%$ \\
\hline & $\mathrm{t} 2 \_\mathrm{i} 4$ & 0.36963 & 0.36872 & 0.02863 & 0.00091 & 0.02 & 0.02703 & 0.00268 & 0.00197 & $100.00 \%$ \\
\hline & $\mathrm{t} 2 \_\mathrm{i} 5$ & 0.23551 & 0.23424 & 0.01759 & 0.00127 & 0.02498 & 0.01743 & 0.00163 & 0.00756 & $100.00 \%$ \\
\hline \multirow{5}{*}{ 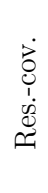 } & i1 & & & & & & & & & \\
\hline & $\mathrm{i} 2$ & 0.01619 & 0.01543 & 0.02009 & 0.00077 & 0.01826 & 0.02022 & 0.00190 & -0.00196 & $11.00 \%$ \\
\hline & i3 & 0.01490 & 0.01488 & 0.01295 & 0.00001 & 0.01421 & 0.01250 & 0.00116 & 0.00172 & $23.60 \%$ \\
\hline & i4 & & & 0.02779 & 0.00034 & 0.02 & & 0.00272 & -0.00207 & $99.60 \%$ \\
\hline & i5 & 0.04074 & 0.04111 & 0.01666 & -0.00036 & 0.01685 & 0.01683 & 0.00161 & 0.00002 & $69.20 \%$ \\
\hline \multirow{5}{*}{ 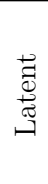 } & $\mathrm{t} 1 \xi$ & & 0.0 & 3715 & & 0.0 & & 11 & 36 & 10.0070 \\
\hline & $\mathrm{t} 2 \xi$ & -0.09821 & -0.03993 & 0.03770 & -0.05828 & 0.03205 & 0.02651 & 0.00234 & 0.00554 & $39.20 \%$ \\
\hline & $\mathrm{t} 1 \Phi$ & 0.06032 & 0.06045 & 0.00969 & -0.00013 & 0.01711 & 0.00945 & 0.00118 & 0.00765 & $100.00 \%$ \\
\hline & $\mathrm{t} 2 \Phi$ & 0.06032 & 0.06045 & 0.00969 & -0.00013 & 0.01711 & 0.00945 & 0.00118 & 0.00765 & $100.00 \%$ \\
\hline & $\mathrm{r}$ & 0.05418 & 0.05433 & 0.01006 & -0.00015 & 0.01738 & 0.00972 & 0.00123 & 0.00766 & $100.00 \%$ \\
\hline
\end{tabular}


Study 3: Psychometric Effects of Applicants' Faking Behaviour

Table 3:

Comparison of the parameter estimates (and SEs) and the parameter estimates of the Monte Carlo simulation study (and SEs), the magnitude of the resulting absolute bias, and percentage of significant parameter estimates for the scale activity in familiar communicative situation in the faking-goodcondition.

\begin{tabular}{|c|c|c|c|c|c|c|c|c|c|c|}
\hline & \multicolumn{4}{|c|}{ Parameter Estimate } & \multicolumn{5}{|c|}{ Parameter Estimate's SF } \\
\hline & & Estimate & M & $\mathrm{SD}$ & Bias & $\mathrm{SE}$ & M & $\mathrm{SD}$ & Bias & Power \\
\hline \multirow{10}{*}{ 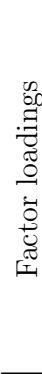 } & t1_i1 & 1 & - & - & 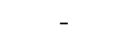 & - & - & - & - & - \\
\hline & t1_i2 & 5 & 19122 & (1) & .00187 & .16116 & 0.13413 & 0.01895 & 0.02704 & $100.00 \%$ \\
\hline & t1_i3 & & 1.18951 & 0.12949 & -0.00931 & 0.12299 & 0.11746 & 0.01765 & 0.00553 & $100.00 \%$ \\
\hline & t1_i4 & & 1.14093 & 0.15460 & -0.01238 & 0.24416 & 0.14491 & 0.01900 & 0.09925 & $100.00 \%$ \\
\hline & t1_i5 & 1.093 & 1.09091 & 0.12734 & 0.00216 & 0.20547 & 0.12316 & 0.01685 & 0.08232 & $100.00 \%$ \\
\hline & t2_i1 & 1 & - & - & 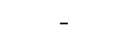 & 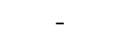 & 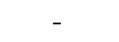 & 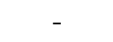 & & 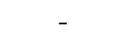 \\
\hline & t2_i2 & .18935 & .19122 & 14316 & .00187 & .16116 & 0.13413 & 0.01895 & 0.02704 & $100.00 \%$ \\
\hline & t2_i3 & & 1.18 & 12949 & -0.00931 & 0.12299 & 0.11746 & 0. & 0.0 & $100.00 \%$ \\
\hline & & & & & 001 & & & & & \\
\hline & t2_i5 & 1.0 & 1.09 & 34 & 0.0 & 0.2 & 0.1 & 0.1 & 0.0 & $00 \%$ \\
\hline \multirow{10}{*}{ 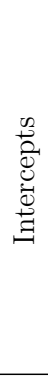 } & ${ }_{0}$ & ; & 81 & & & 0.016 & 9 & & & \\
\hline & t1 & & 2 & & & & & & & \\
\hline & t1_i3 & & & & & & & & -0.0 & $0 \%$ \\
\hline & t1_i4 & & 2.96 & & & & 0.0 & & $-0 .($ & $0 \%$ \\
\hline & $\mathrm{t} 1 \_\mathrm{i}$ & & $356 \Rightarrow$ & & 0.0 & & 0.0 & & -0 & $0 \%$ \\
\hline & t2_i1 & & 3.8 & & & & & & -0 . & \\
\hline & t2_i2 & & 3.29 & & & & & & -0. & \\
\hline & & & & & & & & & -0. & \\
\hline & $\mathrm{t} 2 \_\mathrm{i}$ & & 3.27 & & & & & & & \\
\hline & $\mathrm{t} 2 \_\mathrm{i}$ & & 3.56 & 40 & 0.0 & & 0.0 & & -0. & 100 \\
\hline \multirow{10}{*}{ 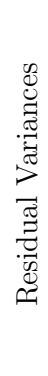 } & & & 0.08954 & & & & & & & \\
\hline & t1_i & & & & & & & & & $0 \%$ \\
\hline & $\mathrm{t} 1 \_\mathrm{i}$ & & & & & & & & & $00 \%$ \\
\hline & t1_i & & & & & & & & & \\
\hline & t1_i5 & & 4 & 9 & & & 0. & & & $0 \%$ \\
\hline & t2_i1 & & & & & & & & & $0 \%$ \\
\hline & t2_i2 & & & & & & & & & $.00 \%$ \\
\hline & t2_i3 & & 0.15889 & & & & & & 0.0 & $100.00 \%$ \\
\hline & t2_i4 & & 0.36872 & & & & & & & $100.00 \%$ \\
\hline & t2_i5 & 0 & 0.23424 & 01750 & 0.00127 & 0.0 & 0.01 & 0.0 & 0.0 & $100.00 \%$ \\
\hline \multirow{5}{*}{ 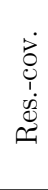 } & te & & & & & & & & & \\
\hline & i2 & & & & & & & & & \\
\hline & i3 & & 0 & 0 & 0.0 & & 0.0 & & & $0 \%$ \\
\hline & i4 & & 0.11549 & & & & 0.02642 & 72 & -0.0 & $99.60 \%$ \\
\hline & i5 & 94074 & 0.04111 & 1666 & -0.00036 & 0.01 & 0.01683 & 0.00161 & 0.00002 & $69.20 \%$ \\
\hline \multirow{5}{*}{ 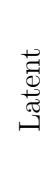 } & $\mathrm{t}$ & & & & & & & & & \\
\hline & t2 & & & & & & & & -0.0 & $70.00 \%$ \\
\hline & t1 $\Phi$ & & & & & & & & & $100.00 \%$ \\
\hline & $\mathrm{t} 2 \Phi$ & & & & & & & & & $100.00 \%$ \\
\hline & $\mathrm{r}$ & 0.00113 & 0.00052 & 0.00998 & 0.00061 & 0.00729 & 0.00980 & 0.00176 & -0.00251 & $6.40 \%$ \\
\hline
\end{tabular}


Study 3: Psychometric Effects of Applicants' Faking Behaviour

Table 4:

Comparison of the parameter estimates (and SEs) and the parameter estimates of the Monte Carlo simulation study (and SEs), the magnitude of the resulting absolute bias, and percentage of significant parameter estimates for the scale preventive health behaviour in response to warning signals in the honest-condition.

\begin{tabular}{|c|c|c|c|c|c|c|c|c|c|c|}
\hline & \multicolumn{4}{|c|}{ Parameter Estimate } & \multicolumn{5}{|c|}{ Parameter Estimate's SE } \\
\hline & & Estimate & M & SD & Bias & $\mathrm{SE}$ & M & $\mathrm{SD}$ & Bias & Power \\
\hline \multirow{10}{*}{ 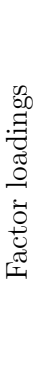 } & t1_i1 & 1 & - & - & - & - & - & - & - & - \\
\hline & $\mathrm{t} 1 \mathrm{i}_{2}$ & 1.31382 & 1.31377 & 0.10743 & 0.00005 & 0.12431 & 0.11189 & 0.01723 & 0.01242 & $100.00 \%$ \\
\hline & t1_i3 & 0.93123 & 0.93173 & 0.08318 & -0.00049 & 0.10629 & 0.08280 & 0.01309 & 0.02350 & $100.00 \%$ \\
\hline & t1_i4 & 0.59878 & 0.59923 & 0.07863 & -0.00045 & 0.10741 & 0.07698 & 0.01043 & 0.03043 & $100.00 \%$ \\
\hline & t1_i5 & 1.18871 & 1.19422 & 0.10298 & -0.00551 & 0.10682 & 0.10400 & 0.01561 & 0.00282 & $100.00 \%$ \\
\hline & t2_i1 & 1 & - & - & - & - & - & - & - & - \\
\hline & $\mathrm{t} 2 \_\mathrm{i} 2$ & 1.31382 & 1.31377 & 0.10743 & 0.00005 & 0.12431 & 0.11189 & 0.01723 & 0.00005 & $100.00 \%$ \\
\hline & $\mathrm{t} 2 \mathrm{i} 3$ & 0.93123 & 0.93173 & 0.08318 & 0.00049 & 0.10629 & 0.08280 & 0.01309 & 0.00049 & $100.00 \%$ \\
\hline & $\mathrm{t} 2 \mathrm{i} 4$ & 0.59878 & 0.59923 & 0.07863 & 0.00045 & 0.10741 & 0.07698 & 0.01043 & 0.00045 & $100.00 \%$ \\
\hline & $\mathrm{t} 2$ _i 5 & 1.18871 & 1.19422 & 0.10298 & 0.00551 & 0.10682 & 0.10400 & 0.01561 & 0.00551 & $100.00 \%$ \\
\hline \multirow{10}{*}{ 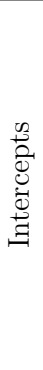 } & t1_i1 & 3.23243 & 3.19750 & 0.04392 & 0.03493 & 0.02566 & 0.02571 & 0.00132 & -0.00005 & $100.00 \%$ \\
\hline & $\mathrm{t} 1$ i 2 & 3.57689 & 3.53352 & 0.04669 & 0.04337 & 0.01960 & 0.02020 & 0.00110 & -0.00061 & $100.00 \%$ \\
\hline & $\mathrm{t} 1 \mathrm{i} 3$ & 3.76497 & 3.73448 & 0.03548 & 0.03049 & 0.01741 & 0.01783 & 0.00089 & -0.00042 & $100.00 \%$ \\
\hline & t1_i4 & 3.68587 & 3.66788 & 0.03454 & 0.01799 & 0.02689 & 0.02667 & 0.00125 & 0.00021 & $100.00 \%$ \\
\hline & t1_i5 & 3.45386 & 3.41485 & 0.04952 & 0.03901 & 0.02404 & 0.02312 & 0.00127 & 0.00092 & $100.00 \%$ \\
\hline & t2_i1 & 3.23243 & 3.19750 & 0.04392 & 0.03493 & 0.02566 & 0.02571 & 0.00132 & -0.00005 & $100.00 \%$ \\
\hline & $\mathrm{t} 2 \mathrm{i} 2$ & 3.57689 & 3.53352 & 0.04669 & 0.04337 & 0.01960 & 0.02020 & 0.00110 & -0.00061 & $100.00 \%$ \\
\hline & $\mathrm{t} 2 \mathrm{i} 3$ & 3.76497 & 3.73448 & 0.03548 & 0.03049 & 0.01741 & 0.01783 & 0.00089 & -0.00042 & $100.00 \%$ \\
\hline & $\mathrm{t} 2$ _i 4 & 3.68587 & 3.66788 & 0.03454 & 0.01799 & 0.02689 & 0.02667 & 0.00125 & 0.00021 & $100.00 \%$ \\
\hline & t2_i5 & 3.45386 & 3.41485 & 0.04952 & 0.03901 & 0.02404 & 0.02312 & 0.00127 & 0.00092 & $100.00 \%$ \\
\hline \multirow{10}{*}{ 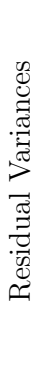 } & t1_i1 & 0 & 0.33027 & 24 & 9 & 0 & 0.02 & 32 & 4 & 100 \\
\hline & t1_i2 & 0.23232 & 0.23072 & 0.02128 & 0.00160 & 0.03366 & 0.02060 & 0.00178 & 0.01306 & $100.00 \%$ \\
\hline & t1_i3 & 0.10358 & 0.10326 & 0.01185 & 0.00031 & 0.01618 & 0.01113 & 0.00113 & 0.00504 & $100.00 \%$ \\
\hline & t1_i4 & 0.23769 & 0.23564 & 0.01710 & 0.00205 & 0.03231 & 0.01771 & 0.00168 & 0.01460 & $100.00 \%$ \\
\hline & $\mathrm{t} 1 \_\mathrm{i} 5$ & 0.20814 & 0.20656 & 0.01821 & 0.00158 & 0.02679 & 0.01810 & 0.00157 & 0.00868 & $100.00 \%$ \\
\hline & t2_i1 & 0.33396 & 0.33027 & 0.02407 & 0.00369 & 0.03459 & 0.02415 & 0.00232 & 0.01044 & $100.00 \%$ \\
\hline & $\mathrm{t} 2 \_\mathrm{i} 2$ & 0.23232 & 0.23072 & 0.02128 & 0.00160 & 0.03366 & 0.02060 & 0.00178 & 0.01306 & $100.00 \%$ \\
\hline & $\mathrm{t} 2 \_\mathrm{i} 3$ & 0.23605 & 0.23584 & 0.03088 & 0.00021 & 0.02468 & 0.02890 & 0.00417 & -0.00422 & $100.00 \%$ \\
\hline & t2_i4 & 0.38574 & 0.38184 & 0.06269 & 0.00390 & 0.06443 & 0.05847 & 0.01223 & 0.00596 & $100.00 \%$ \\
\hline & t2_i5 & 0.20814 & 0.20656 & 0.01821 & 0.00158 & 0.02679 & 0.01810 & 0.00157 & 0.00868 & $100.00 \%$ \\
\hline \multirow{5}{*}{ 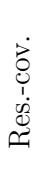 } & i1 & 0.07630 & 0.07367 & 0.02363 & 0.00263 & 0.02694 & 0.02373 & 0.00221 & 321 & $88.80 \%$ \\
\hline & i2 & 0.04014 & 0.03848 & 0.01950 & 0.00166 & 0.02361 & 0.01952 & 0.00175 & 0.00410 & $48.80 \%$ \\
\hline & i3 & 0.03352 & 0.03271 & 0.01106 & 0.00081 & 0.01395 & 0.01078 & 0.00106 & 0.00316 & $88.20 \%$ \\
\hline & i4 & 0.06629 & 0.06413 & 0.01718 & 0.00216 & 0.01803 & 0.01717 & 0.00154 & 0.00087 & $97.20 \%$ \\
\hline & i5 & 0.03724 & 0.03632 & 0.01852 & 0.00092 & 0.02124 & 0.01712 & 0.00159 & 0.00412 & $54.40 \%$ \\
\hline \multirow{5}{*}{ 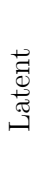 } & $\mathrm{t} 1 \xi$ & 0.00485 & 0.03754 & 0.03109 & -0.03269 & 0.02176 & 0.02292 & 0.00166 & -0.00116 & $39.60 \%$ \\
\hline & $\mathrm{t} 2 \xi$ & -0.29433 & -0.25980 & 0.03868 & -0.03453 & 0.03817 & 0.03695 & 0.00252 & 0.00122 & $100.00 \%$ \\
\hline & $\mathrm{t} 1 \Phi$ & 0.12546 & 0.12563 & 0.02088 & -0.00017 & 0.02414 & 0.02035 & 0.00261 & 0.00379 & $100.00 \%$ \\
\hline & $\mathrm{t} 2 \Phi$ & 0.12546 & 0.12563 & 0.02088 & -0.00017 & 0.02414 & 0.02035 & 0.00261 & 0.00379 & $100.00 \%$ \\
\hline & $\mathrm{r}$ & 0.04859 & 0.04872 & 0.01884 & -0.00013 & 0.02403 & 0.01816 & 0.00321 & 0.00587 & $79.00 \%$ \\
\hline
\end{tabular}


Study 3: Psychometric Effects of Applicants' Faking Behaviour

Table 5:

Comparison of the parameter estimates (and SEs) and the parameter estimates of the Monte Carlo simulation study (and SEs), the magnitude of the resulting absolute bias, and percentage of significant parameter estimates for the scale preventive health behaviour in response to warning signals in the reproduce-condition.

\begin{tabular}{|c|c|c|c|c|c|c|c|c|c|c|}
\hline & \multicolumn{4}{|c|}{ Parameter Estimate } & \multicolumn{5}{|c|}{ Parameter Estimate's SE } \\
\hline & & Estimate & M & $\mathrm{SD}$ & Bias & $\mathrm{SE}$ & M & $\mathrm{SD}$ & Bias & ower \\
\hline \multirow{10}{*}{ 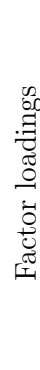 } & t1_i1 & 1 & - & - & - & - & - & - & - & \\
\hline & $\mathrm{t} 1 \mathrm{i}_{2}$ & .31382 & 1.31377 & 10743 & 0.00005 & 12431 & 0.11189 & 0.01723 & 0.01242 & $100.00 \%$ \\
\hline & $\mathrm{t} 1$ i 3 & & 0.93173 & 0.08318 & -0.00049 & 0.10629 & 0.08280 & .01309 & 0.02350 & $100.00 \%$ \\
\hline & t1_i4 & 0.59878 & 0.59923 & 0.07863 & -0.00045 & 0.10741 & 0.07698 & 0.01043 & 0.03043 & $100.00 \%$ \\
\hline & $\mathrm{t} 1 \_\mathrm{i} 5$ & 1.18871 & 1.19422 & 0.10298 & -0.00551 & 0.10682 & 0.10400 & 0.01561 & 0.00282 & $100.00 \%$ \\
\hline & t2_i1 & 1 & - & - & - & - & - & - & - & - \\
\hline & $\mathrm{t} 2 \mathrm{i} 2$ & 1.31382 & 1.31377 & 0.10743 & 0.00005 & 0.12431 & 0.11189 & 0.01723 & 0.01242 & $100.00 \%$ \\
\hline & $\mathrm{t} 2 \mathrm{i} 3$ & 0.93123 & 0.93173 & 0.08318 & -0.00049 & 0.10629 & 0.08280 & 0.01309 & 0.02350 & $100.00 \%$ \\
\hline & $\mathrm{t} 2 \mathrm{i} 4$ & 0.59878 & 0.59923 & 0.07863 & -0.00045 & 0.10741 & 0.07698 & 0.01043 & 0.03043 & $100.00 \%$ \\
\hline & $\mathrm{t} 2 \_\mathrm{i} 5$ & 1.18871 & 1.19422 & 0.10298 & -0.00551 & 0.10682 & 0.10400 & 0.01561 & 0.00282 & $100.00 \%$ \\
\hline \multirow{10}{*}{ 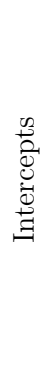 } & t1_i & 3.23243 & 3.19 & 2 & 0. & 02 & 0.02 & 32 & 5 & $100.00 \%$ \\
\hline & $\mathrm{t} 1$ i 2 & & 3.5 & & & & 0.02 & & 61 & $0 \%$ \\
\hline & $\mathrm{t} 1 \mathrm{i} 3$ & & 3.7 & & & & 0.01 & & -0.1 & 100 \\
\hline & t1_i4 & & 3.6 & & & 0.0 & 0.02 & & & $100.00 \%$ \\
\hline & t1_i5 & & 3.4 & & & & 0.02 & & & $100.00 \%$ \\
\hline & $\mathrm{t} 2 \mathrm{i} 1$ & & 3.1 & 0 & & 66 & 0.02571 & 32 & -0.00 & $100.00 \%$ \\
\hline & $\mathrm{t} 2 \_\mathrm{i} 2$ & & 3.5 & 0.04669 & & 001 & 0.02020 & 110 & -0.0 & $100.00 \%$ \\
\hline & $\mathrm{t} 2 \mathrm{i} 3$ & & 3.7 & & & 41 & 0.01783 & & -0.0 & $100.00 \%$ \\
\hline & $\mathrm{t} 2$ i 4 & & 3.4 & 0.0 & & 99 & 0.05 & & & $100.00 \%$ \\
\hline & $\mathrm{t} 2 \_\mathrm{i} 5$ & 3.4 & 3.41 & 0.04952 & & 0.0 & 0.02312 & 27 & & $100.00 \%$ \\
\hline \multirow{10}{*}{ 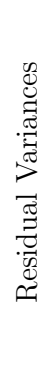 } & & & & & & & & & & \\
\hline & t1_i2 & & & & & & & & & 10 \\
\hline & $\mathrm{t} 1$ i $\mathrm{i} 3$ & & & & & & 0. & & 0.1 & 10 \\
\hline & t1_i4 & 9 & 0.23 & 0.01 & 0.0 & 31 & 0.01 & 68 & 0.0 & $100.00 \%$ \\
\hline & $\mathrm{t} 1$ i 5 & P & 0.20656 & 0.01821 & 58 & 0.02679 & 0.01810 & 157 & 0.0 & $100.00 \%$ \\
\hline & t2_i1 & & & & & & 0. & & & $100.00 \%$ \\
\hline & $\mathrm{t} 2 \mathrm{i} 2$ & & 0.23072 & 0.02128 & & 0.0 & 0.02060 & 178 & 0.01306 & $100.00 \%$ \\
\hline & $\mathrm{t} 2 \_\mathrm{i} 3$ & 0.23605 & 0.23584 & 0.03088 & 0.00021 & 0.02468 & 0.02890 & 0.00417 & -0.00422 & $100.00 \%$ \\
\hline & t2_i4 & 0.23769 & 0.23564 & 0.01710 & 0.00205 & 0.03231 & 0.01771 & 0.00168 & 0.01460 & $100.00 \%$ \\
\hline & $\mathrm{t} 2 \mathrm{i} 5$ & 0.20814 & 0.20656 & 0.01821 & 0.00158 & 0.02679 & 0.01810 & 0.00157 & 0.00868 & $100.00 \%$ \\
\hline \multirow{5}{*}{$\begin{array}{c}\dot{0} \\
\dot{0} \\
1 \\
\dot{d} \\
\dot{D}\end{array}$} & i1 & & & & & & & & & \\
\hline & i2 & $t$ & & 50 & 6 & 1 & 0.0 & 75 & 0.0 & $30 \%$ \\
\hline & i3 & 0.03352 & 0.03271 & 0.01106 & 0.00 & 0.01 & 0.01078 & 0.0 & 0.0 & $88.20 \%$ \\
\hline & i4 & 0.06629 & 0.06413 & 0.01718 & 0.00216 & $0.01 \S$ & 0.01717 & 0.00154 & 0.00087 & $97.20 \%$ \\
\hline & i5 & 0.03724 & 0.03632 & 0.01852 & 0.00092 & 0.02124 & 0.01712 & 0.00159 & 0.00412 & $54.40 \%$ \\
\hline \multirow{5}{*}{ 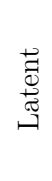 } & $\mathrm{t} 1 \xi$ & & & & & & & & & $39.60 \%$ \\
\hline & $\mathrm{t} 2 \xi$ & -0.29433 & -0.25980 & 0.03868 & -0.03453 & 0.03817 & 0.03695 & 0.00252 & 0.00122 & $100.00 \%$ \\
\hline & $\mathrm{t} 1 \Phi$ & 0.12546 & 0.12563 & 0.02088 & -0.00017 & 0.02414 & 0.02035 & 0.00261 & 0.00379 & $100.00 \%$ \\
\hline & $\mathrm{t} 2 \Phi$ & 0.279 & 0.283 & 0.06074 & -0.00408 & 0.05993 & 0.06003 & 0.01211 & -0.00009 & $100.00 \%$ \\
\hline & $\mathrm{r}$ & 0.13089 & 0.13312 & 0.02808 & -0.00223 & 0.03316 & 0.02826 & 0.00443 & 0.00491 & $100.00 \%$ \\
\hline
\end{tabular}


Study 3: Psychometric Effects of Applicants' Faking Behaviour

Table 6:

Comparison of the parameter estimates (and SEs) and the parameter estimates of the Monte Carlo simulation study (and SEs), the magnitude of the resulting absolute bias, and percentage of significant parameter estimates for the scale preventive health behaviour in response to warning signals in the faking-good-condition.

\begin{tabular}{|c|c|c|c|c|c|c|c|c|c|c|}
\hline & \multicolumn{4}{|c|}{ Parameter Estimate } & \multicolumn{5}{|c|}{ Parameter Estimate's SE } \\
\hline & & & M & $\mathrm{SD}$ & ias & $\mathrm{SE}$ & M & $\mathrm{SD}$ & Bias & Power \\
\hline \multirow{10}{*}{ 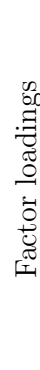 } & & 1 & - & - & - & - & 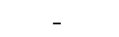 & 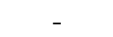 & & 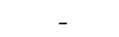 \\
\hline & $\mathrm{t}$ & 2 & 31377 & 07 & 000 & 124 & 11189 & $-7+2$ & 2 & 0.00 \\
\hline & t1_i3 & & 93173 & 8 & .00 & 9 & 8280 & 9 & & 70 \\
\hline & t1_i4 & 878 & 59923 & 07863 & .00045 & 10741 & 0.07698 & 01043 & 43 & $00.00 \%$ \\
\hline & t1_i5 & & 19422 & & & 32 & 0.10400 & & & $00.00 \%$ \\
\hline & t2_i1 & 1 & - & - & - & - & - & - & - & - \\
\hline & $\mathrm{t} 2 \_\mathrm{i} 2$ & .31382 & 7 & & 55 & & & & 0.01242 & $100.00 \%$ \\
\hline & $\mathrm{t} 2 \mathrm{i} 3$ & & .93173 & & & & & & & $100.00 \%$ \\
\hline & t2_i4 & & 23 & & & & & & & $100.00 \%$ \\
\hline & $\mathrm{t} 2 \_\mathrm{i} 5$ & 1.18871 & 1.19422 & 0298 & 51 & 0.10682 & 0.10400 & 61 & 0.0 & $100.00 \%$ \\
\hline \multirow{10}{*}{ 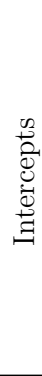 } & t. & 323243 & 319750 & 0.04302 & 0,03403 & 0.02566 & 0.02571 & & & $100.00 \%$ \\
\hline & & & & & & & & & & \\
\hline & & & & & & & & & & \\
\hline & & & & & & & & & & \\
\hline & t1_i5 & & & & & & & & & \\
\hline & t2_i1 & & & & & & & & & $00 \%$ \\
\hline & $\mathrm{t} 2 \_\mathrm{i} 2$ & & & & & & & & & \\
\hline & $\mathrm{t} 2 \mathrm{i} 3$ & & 272 & & & & & & & \\
\hline & $\mathrm{t} 2$ i 4 & & & & & & & & & $.00 \%$ \\
\hline & & & 250 & & & & & & 9 & 07 \\
\hline \multirow{10}{*}{ 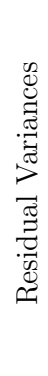 } & & & & & & & & & & \\
\hline & t1_i2 & & & & & & & & & \\
\hline & t1_i3 & & & & & & & & & $0 \%$ \\
\hline & t1_i4 & & 0.23564 & & & & & & & $00 \%$ \\
\hline & t1_i5 & & 0.20656 & & & & & & & $.00 \%$ \\
\hline & t2_i1 & & & & & & & & & $00 \%$ \\
\hline & t2_i2 & & 0.23072 & & & & & & & $100.00 \%$ \\
\hline & $\mathrm{t} 2 \mathrm{i} 3$ & & 0.10326 & & & & & & & $100.00 \%$ \\
\hline & t2_i4 & & & & & & & & & $100.00 \%$ \\
\hline & t2_i5 & 0.20814 & 0.20656 & 0.01821 & 0.00158 & 0.02679 & 0.01810 & 0.00157 & 0.00868 & $100.00 \%$ \\
\hline \multirow{5}{*}{$\begin{array}{c}\overrightarrow{0} \\
\dot{0} \\
1 \\
\dot{d} \\
\dot{d}\end{array}$} & 11 & & & & & & & & & $0 \%$ \\
\hline & i2 & & 0.03848 & & & & & & & $80 \%$ \\
\hline & i3 & & 0.03271 & & & & & & & $8.20 \%$ \\
\hline & i4 & & 0.06413 & & ק & & & & 0.00087 & $97.20 \%$ \\
\hline & i5 & 0.03724 & 0.03632 & 0.01852 & 0.00092 & 0.02124 & 0.01712 & 0.00159 & 0.00412 & $54.40 \%$ \\
\hline \multirow{5}{*}{ 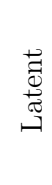 } & & & & & & & & & & \\
\hline & $\mathrm{t} 2 \xi$ & 0.1008 & 0.15004 & & & & & & & \\
\hline & $\mathrm{t} 1 \Phi$ & 0.12546 & 0.12563 & 0.02088 & -0.00017 & 0.02414 & 0.02035 & 0.00261 & 0.00379 & $100.00 \%$ \\
\hline & $\mathrm{t} 2 \Phi$ & 0.12546 & 0.12563 & 0.02088 & -0.00017 & 0.02414 & 0.02035 & 0.00261 & 0.00379 & $100.00 \%$ \\
\hline & $\mathrm{r}$ & 0.04960 & 0.05000 & 0.01813 & -0.00040 & 0.01975 & 0.01755 & 0.00303 & 0.00220 & $82.80 \%$ \\
\hline
\end{tabular}


Study 3: Psychometric Effects of Applicants' Faking Behaviour

Table 7:

Comparison of the parameter estimates (and SEs) and the parameter estimates of the Monte Carlo simulation study (and SEs), the magnitude of the resulting absolute bias, and percentage of significant parameter estimates for the scale self-confidence in test situation in the honest-condition.

\begin{tabular}{|c|c|c|c|c|c|c|c|c|c|c|}
\hline & \multicolumn{4}{|c|}{ Parameter Estimate } & \multicolumn{5}{|c|}{ Parameter Estimate's SE } \\
\hline & & Estimat & M & $\mathrm{SD}$ & Bias & $\mathrm{SE}$ & M & $\mathrm{SD}$ & Bias & ower \\
\hline \multirow{10}{*}{ 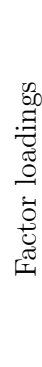 } & t1_i1 & 1 & - & - & - & - & - & . & - & . \\
\hline & t1_i2 & 1.127 & $1.12 \mathrm{~s}$ & 0.05648 & -0.00229 & .05632 & 0.05622 & 0.00519 & 0.00010 & $100.00 \%$ \\
\hline & $\mathrm{t} 1 \_\mathrm{i} 3$ & 1.04328 & 1.04417 & 0.07201 & -0.00090 & 0.06807 & 0.06963 & 0.00636 & -0.00156 & $100.00 \%$ \\
\hline & t1_i4 & 0.78068 & 0.78300 & 0.05294 & -0.00232 & 0.05091 & 0.04774 & 0.00416 & 0.00317 & $100.00 \%$ \\
\hline & t1_i5 & 0.79442 & 0.79364 & 0.06397 & 0.00078 & 0.05484 & 0.06335 & 0.00636 & -0.00851 & $100.00 \%$ \\
\hline & t2_i1 & 1 & - & - & - & - & - & - & - & - \\
\hline & t2_i2 & 1.12711 & 1.12940 & 0.05648 & -0.00229 & 0.05632 & 0.05622 & 0.00519 & 0.00010 & $100.00 \%$ \\
\hline & t2_i3 & 1.04328 & 1.04417 & 0.07201 & -0.00090 & 0.06807 & 0.06963 & 0.00636 & -0.00156 & $100.00 \%$ \\
\hline & t2_i4 & 0.78068 & 0.78300 & 0.05294 & -0.00232 & 0.05091 & 0.04774 & 0.00416 & 0.00317 & $100.00 \%$ \\
\hline & t2_i 5 & 0.79442 & 0.79364 & 0.06397 & 0.00078 & 0.05484 & 0.06335 & 0.00636 & -0.00851 & $100.00 \%$ \\
\hline \multirow{10}{*}{ 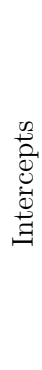 } & t1_i1 & 3.98927 & 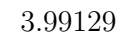 & 1 & -0.00202 & 2 & 0.0 & 91 & -0.0 & $9 \%$ \\
\hline & & & & 0.10 & -0.0 & & 0.09999 & 59 & -0.0 & $100.00 \%$ \\
\hline & t1_i3 & & & 0.10 & -0.0 & & 0.11082 & & -0.0 & $100.00 \%$ \\
\hline & t1_i4 & & 3.9 & & & & 0.07 & 0.02225 & -0.0 & $100.00 \%$ \\
\hline & t1_i5 & 4.0 & 4.00752 & 0.11 & -0.00 & 44 & 0.1 & & -0.0 & $100.00 \%$ \\
\hline & t2_i1 & 3.98927 & 3.99129 & 0.09231 & -0.00202 & 0.07452 & 0.08796 & 0.02891 & -0.01 & $100.00 \%$ \\
\hline & t2_i2 & 3.95941 & 3.96224 & 0.10338 & -0.00284 & 0.08166 & 0.09999 & 0.03259 & -0.01833 & $100.00 \%$ \\
\hline & t2_i3 & 3.72510 & 3.72720 & 0.10942 & -0.00210 & 55 & 0.11082 & 3077 & -0.00 & $100.00 \%$ \\
\hline & t2_i4 & 3.93 & 3.93 & 0.07321 & 0.00 & 58 & 0.07040 & 0.02225 & -0.01 & $100.00 \%$ \\
\hline & $\mathrm{t} 2 \mathrm{i} 5$ & 3.83 & 3.83 & 0.11 & -0.00314 & 88 & & 0.03168 & -0.00 & $100.00 \%$ \\
\hline \multirow{10}{*}{ 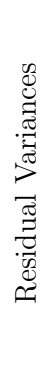 } & & & & & & & & & & \\
\hline & t1_i & 4 & 0.2 & 0.0 & & 71 & 0.02 & 201 & 0.0 & $00 \%$ \\
\hline & t1_i & 33 & 0.2 & 0.02 & 0.0 & 06 & 0.02361 & 226 & 0.0 & $100.00 \%$ \\
\hline & t1_i4 & & 0.2 & 0.0 & 4 & 33 & 0.0 & 91 & 0.0 & $100.00 \%$ \\
\hline & t1_i5 & 0.22 & 0.2 & 0.01 & 9 & $002-2$ & 0.01776 & 0.00177 & 0.0 & $100.00 \%$ \\
\hline & t2_i1 & 0.17 & 0.17 & 0.016 & & & 0.01635 & 55 & 0.0 & $100.00 \%$ \\
\hline & t2_i2 & 0.23764 & 0.23692 & 0.02164 & 0.00072 & 0.02171 & 0.02160 & 0.00201 & 0.00010 & $100.00 \%$ \\
\hline & t2_i3 & 0.27983 & 0.27791 & 0.02379 & 0.00192 & 0.02506 & 0.02361 & 0.00226 & 0.00145 & $100.00 \%$ \\
\hline & t2_i4 & 0.27216 & 0.27052 & 0.02099 & 0.00164 & 0.02723 & 0.02072 & 0.00191 & 0.00651 & $100.00 \%$ \\
\hline & $\mathrm{t} 2 \mathrm{i} 5$ & 0.22238 & 0.22128 & 0.01899 & 0.00109 & 0.02189 & 0.01776 & 0.00177 & 0.00413 & $100.00 \%$ \\
\hline \multirow{5}{*}{$\begin{array}{l}\overrightarrow{0} \\
1 \\
1 \\
\dot{D} \\
\dot{0}\end{array}$} & i1 & & & & & & 0 & & & $6.80^{\circ}$ \\
\hline & i2 & & & 0. & & & 0.0 & 57 & -0 . & $30.00 \%$ \\
\hline & i3 & & 0.0 & 0.017 & 0.00 & 0.02099 & 0.0 & 0.00167 & 0.00 & $66.80 \%$ \\
\hline & i4 & 0.02354 & 0.02350 & 0.01237 & 0.00003 & 0.01512 & 0.01219 & 0.00135 & 0.00294 & $46.80 \%$ \\
\hline & i5 & 0.01786 & 0.01807 & 0.00949 & -0.00021 & 0.00974 & 0.00901 & 0.00112 & 0.00073 & $50.20 \%$ \\
\hline \multirow{5}{*}{ 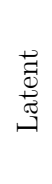 } & $\mathrm{t} 1 \xi$ & & & & & & & & & $100.00 \%$ \\
\hline & $\mathrm{t} 2 \xi$ & -0.82012 & -0.82327 & 0.11419 & 0.00314 & 0.11005 & 0.11436 & 0.02524 & -0.00431 & $100.00 \%$ \\
\hline & t1 $\Phi$ & 0.19298 & 0.19302 & 0.02353 & -0.00004 & 0.02416 & 0.02280 & 0.00282 & 0.00136 & $100.00 \%$ \\
\hline & $\mathrm{t} 2$ & 0.40326 & 0.39821 & 0.07160 & 0.00504 & 0.05901 & 0.07193 & 0.01491 & -0.01292 & $100.00 \%$ \\
\hline & $\mathrm{r}$ & 0.14863 & 0.14975 & 0.03800 & -0.00112 & 0.03283 & 0.03550 & 0.00573 & -0.00268 & $98.40 \%$ \\
\hline
\end{tabular}


Study 3: Psychometric Effects of Applicants' Faking Behaviour

Table 8:

Comparison of the parameter estimates (and SEs) and the parameter estimates of the Monte Carlo simulation study (and SEs), the magnitude of the resulting absolute bias, and percentage of significant parameter estimates for the scale self-confidence in test situation in the reproduce-condition.

\begin{tabular}{|c|c|c|c|c|c|c|c|c|c|c|}
\hline & \multicolumn{4}{|c|}{ Parameter Estimate } & \multicolumn{5}{|c|}{ Parameter Estimate's SE } \\
\hline & & Estimate & M & $\mathrm{SD}$ & Bias & SE & M & $\mathrm{SD}$ & Bias & Power \\
\hline \multirow{10}{*}{ 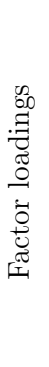 } & t1_i1 & 1 & - & - & - & - & - & - & - & - \\
\hline & $\mathrm{t} 1 \mathrm{i}_{2}$ & 1.12711 & 1.12940 & 0.05648 & -0.00229 & 0.05632 & 0.05622 & 0.00519 & 0.00010 & $100.00 \%$ \\
\hline & $\mathrm{t} 1 \_\mathrm{i} 3$ & 1.04328 & 1.04417 & 0.07201 & -0.00090 & 0.06807 & 0.06963 & 0.00636 & -0.00156 & $100.00 \%$ \\
\hline & t1_i4 & 0.78068 & 0.78300 & 0.05294 & -0.00232 & 0.05091 & 0.04774 & 0.00416 & 0.00317 & $100.00 \%$ \\
\hline & $\mathrm{t} 1 \_\mathrm{i} 5$ & 0.79442 & 0.79364 & 0.06397 & 0.00078 & 0.05484 & 0.06335 & 0.00636 & -0.00851 & $100.00 \%$ \\
\hline & t2_i1 & 1 & - & - & - & - & - & - & - & - \\
\hline & $\mathrm{t} 2 \_\mathrm{i} 2$ & 1.12711 & 1.12940 & 0.05648 & -0.00229 & 0.05632 & 0.05622 & 0.00519 & 0.00010 & $100.00 \%$ \\
\hline & $\mathrm{t} 2 \_\mathrm{i} 3$ & 1.04328 & 1.04417 & 0.07201 & -0.00090 & 0.06807 & 0.06963 & 0.00636 & -0.00156 & $100.00 \%$ \\
\hline & t2_i4 & 0.78068 & 0.78300 & 0.05294 & -0.00232 & 0.05091 & 0.04774 & 0.00416 & 0.00317 & $100.00 \%$ \\
\hline & t2_i5 & 0.79442 & 0.79364 & 0.06397 & 0.00078 & 0.05484 & 0.06335 & 0.00636 & -0.00851 & $100.00 \%$ \\
\hline \multirow{10}{*}{ 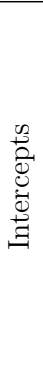 } & t1_i1 & 3.98927 & 3.99129 & 0.09231 & -0.00202 & 0.07452 & 0.08796 & 0.02891 & -0.01344 & $100.00 \%$ \\
\hline & $\mathrm{t} 1$ i2 2 & 3.95941 & 3.96224 & 0.10338 & -0.00284 & 0.08166 & 0.09999 & 0.03259 & -0.01833 & $100.00 \%$ \\
\hline & t1_i3 & 3.72510 & 3.72720 & 0.10942 & -0.00210 & 0.10155 & 0.11082 & 0.03077 & -0.00927 & $100.00 \%$ \\
\hline & t1_i4 & 3.93596 & 3.93576 & 0.07321 & 0.00020 & 0.05958 & 0.07040 & 0.02225 & -0.01082 & $100.00 \%$ \\
\hline & t1_i5 & 4.00351 & 4.00752 & 0.11853 & -0.00401 & 0.09644 & 0.11348 & 0.03148 & -0.01705 & $100.00 \%$ \\
\hline & t2_i1 & 3.98927 & 3.99129 & 0.09231 & -0.00202 & 0.07452 & 0.08796 & 0.02891 & -0.01344 & $100.00 \%$ \\
\hline & t2_i2 & 3.95941 & 3.96224 & 0.10338 & -0.00284 & 0.08166 & 0.09999 & 0.03259 & -0.01833 & $100.00 \%$ \\
\hline & t2_i3 & 3.72510 & 3.72720 & 0.10942 & -0.00210 & 0.10155 & 0.11082 & 0.03077 & -0.00927 & $100.00 \%$ \\
\hline & t2_i4 & 3.93596 & 3.93576 & 0.07321 & 0.00020 & 0.05958 & 0.07040 & 0.02225 & -0.01082 & $100.00 \%$ \\
\hline & $\mathrm{t} 2 \_\mathrm{i} 5$ & 3.83657 & 3.83971 & 0.11926 & -0.00314 & 0.10488 & 0.11370 & 0.03168 & -0.00882 & $100.00 \%$ \\
\hline \multirow{10}{*}{ 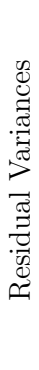 } & i & 0 & 0.17 & 0 & 8 & 9 & 0.016 & 65 & 0.0 & $100.00 \%$ \\
\hline & t1_i2 & 0.23764 & 0.23692 & 0.02164 & 0.00072 & 0.02171 & 0.02160 & 0.00201 & 0.00010 & $100.00 \%$ \\
\hline & t1_i3 & 0.27983 & 0.27791 & 0.02379 & 0.00192 & 0.02506 & 0.02361 & 0.00226 & 0.00145 & $100.00 \%$ \\
\hline & t1_i4 & 0.27216 & 0.27052 & 0.02099 & 0.00164 & 0.02723 & 0.02072 & 0.00191 & 0.00651 & $100.00 \%$ \\
\hline & $\mathrm{t} 1 \mathrm{i}_{1} 5$ & 0.22238 & 0.22128 & 0.01899 & 0.00109 & 0.02189 & 0.01776 & 0.00177 & 0.00413 & $100.00 \%$ \\
\hline & t2_i1 & 0.17638 & 0.17580 & 0.01653 & 0.00058 & 0.01709 & 0.01635 & 0.00155 & 0.00074 & $100.00 \%$ \\
\hline & t2_i2 & 0.23764 & 0.23692 & 0.02164 & 0.00072 & 0.02171 & 0.02160 & 0.00201 & 0.00010 & $100.00 \%$ \\
\hline & t2_i3 & 0.27983 & 0.27791 & 0.02379 & 0.00192 & 0.02506 & 0.02361 & 0.00226 & 0.00145 & $100.00 \%$ \\
\hline & t2_i4 & 0.27216 & 0.27052 & 0.02099 & 0.00164 & 0.02723 & 0.02072 & 0.00191 & 0.00651 & $100.00 \%$ \\
\hline & t2_i 5 & 0.22238 & 0.22128 & 0.01899 & 0.00109 & 0.02189 & 0.01776 & 0.00177 & 0.00413 & $100.00 \%$ \\
\hline \multirow{5}{*}{$\begin{array}{l}\overrightarrow{0} \\
\dot{0} \\
\dot{0} \\
\dot{0} \\
\text { ح| }\end{array}$} & i1 & 0.00083 & 0.00116 & 0.01011 & 0.00033 & 0.00772 & 0.00970 & 0105 & -0.0 & $6.80 \%$ \\
\hline & i2 & 0.02003 & 0.01912 & 0.01463 & 0.00090 & 0.01306 & 0.01372 & 0.00157 & -0.00066 & $30.00 \%$ \\
\hline & i3 & 0.04185 & 0.04073 & 0.01719 & 0.00112 & 0.02099 & 0.01730 & 0.00167 & 0.00369 & $66.80 \%$ \\
\hline & i4 & 0.02354 & 0.02350 & 0.01237 & 0.00003 & 0.01512 & 0.01219 & 0.00135 & 0.00294 & $46.80 \%$ \\
\hline & i5 & 0.01786 & 0.01807 & 0.00949 & -0.00021 & 0.00974 & 0.00901 & 0.00112 & 0.00073 & $50.20 \%$ \\
\hline \multirow{5}{*}{ 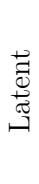 } & t1 $\xi$ & -0.56906 & -0.57280 & 0.10171 & 0.00374 & 0.08634 & 0.09758 & 0.02795 & -0.01124 & $100.00 \%$ \\
\hline & t2 $\xi$ & -0.68493 & -0.68707 & 0.10662 & 0.00214 & 0.09935 & 0.10504 & 0.02656 & -0.00569 & $100.00 \%$ \\
\hline & $\mathrm{t} 1 \Phi$ & 0.19298 & 0.19302 & 0.02353 & -0.00004 & 0.02416 & 0.02280 & 0.00282 & 0.00136 & $100.00 \%$ \\
\hline & t2 $\Phi$ & 0.19298 & 0.19302 & 0.02353 & -0.00004 & 0.02416 & 0.02280 & 0.00282 & 0.00136 & $100.00 \%$ \\
\hline & $\mathrm{r}$ & 0.08534 & 0.08445 & 0.02601 & 0.00089 & 0.02575 & 0.02511 & 0.00372 & 0.00065 & $93.00 \%$ \\
\hline
\end{tabular}


Study 3: Psychometric Effects of Applicants' Faking Behaviour

Table 9:

Comparison of the parameter estimates (and SEs) and the parameter estimates of the Monte Carlo simulation study (and SEs), the magnitude of the resulting absolute bias, and percentage of significant parameter estimates for the scale self-confidence in test situation in the faking-good-condition.

\begin{tabular}{|c|c|c|c|c|c|c|c|c|c|c|}
\hline & \multicolumn{4}{|c|}{ Parameter Estimate } & \multicolumn{5}{|c|}{ Parameter Estimate's SE } \\
\hline & & Esti & M & $\mathrm{SD}$ & Bias & $\mathrm{SE}$ & M & $\mathrm{SD}$ & Bias & ower \\
\hline \multirow{10}{*}{ 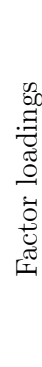 } & t1_i1 & 1 & - & - & - & - & - & - & - & - \\
\hline & $\mathrm{t} 1 \_\mathrm{i} 2$ & 1.12711 & 1.12940 & 0.05648 & -0.00229 & 05632 & 0.05622 & 0.00519 & 0.00010 & $100.00 \%$ \\
\hline & $\mathrm{t} 1$ _i3 & 1.04328 & 1.04417 & 0.07201 & -0.00090 & 0.06807 & 0.06963 & 0.00636 & -0.00156 & $100.00 \%$ \\
\hline & t1_i4 & 0.78068 & 0.78300 & 0.05294 & -0.00232 & 0.05091 & 0.04774 & 0.00416 & 0.00317 & $100.00 \%$ \\
\hline & t1_i5 & 0.79442 & 0.79364 & 0.06397 & 0.00078 & 0.05484 & 0.06335 & 0.00636 & -0.00851 & $100.00 \%$ \\
\hline & t2_i1 & 1 & - & - & - & - & - & - & - & - \\
\hline & t2_i2 & 1.12711 & 1.12940 & 0.05648 & -0.00229 & 0.05632 & 0.05622 & 0.00519 & 0.00010 & $100.00 \%$ \\
\hline & $\mathrm{t} 2$ il3 & 1.04328 & 1.04417 & 0.07201 & -0.00090 & 0.06807 & 0.06963 & 0.00636 & -0.00156 & $100.00 \%$ \\
\hline & $\mathrm{t} 2 \_\mathrm{i} 4$ & 0.78068 & 0.78300 & 0.05294 & -0.00232 & 0.05091 & 0.04774 & 0.00416 & 0.00317 & $100.00 \%$ \\
\hline & $\mathrm{t} 2 \_\mathrm{i} 5$ & 1.43143 & 1.44374 & 0.12409 & -0.01231 & 0.12817 & 0.11788 & 0.02602 & 0.01029 & $100.00 \%$ \\
\hline \multirow{10}{*}{ 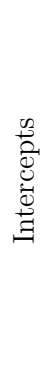 } & 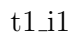 & 3.98927 & 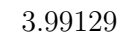 & 1 & -0.0 & 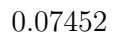 & 6 & 91 & -0.0 & 100 \\
\hline & & & 3.9 & & -0.0 & 6 & 0.09 & & -0.0 & $00 \%$ \\
\hline & t1_i3 & & & & -0.0 & & 0.11 & & -0.0 & $100.00 \%$ \\
\hline & t1_i4 & & 3.9 & & & & & 0.02225 & -0.01 & $100.00 \%$ \\
\hline & t1_i5 & & 4.0 & 0.1 & -0.0 & & & & -0.0 & $100.00 \%$ \\
\hline & t2_i1 & 3.98927 & 3.99129 & 0.09231 & -0.00 & 0.07 & 0.0 & 891 & -0.01 & $100.00 \%$ \\
\hline & t2_i2 & 3.95941 & 3.96224 & 0.10338 & -0.00284 & 0.08166 & 0.09999 & 0.03259 & -0.01 & $100.00 \%$ \\
\hline & t2_i3 & 3.88115 & 3.88505 & 0.09766 & -0.0 & 89 & 0.09489 & 3000 & -0.0 & $100.00 \%$ \\
\hline & t2_i4 & 3.93 & 3.93 & 0.07321 & 0.00 & 58 & $0.0^{\circ}$ & 0.02225 & -0.01 & $100.00 \%$ \\
\hline & t2_i5 & 4.00 & $4.00^{\circ}$ & 0.11 & -0.00 & & & & -0.01 & $100.00 \%$ \\
\hline \multirow{10}{*}{ 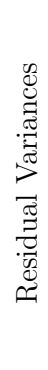 } & & & & & & & & & & \\
\hline & t1_i2 & & 0 & 4 & 0.0 & & & 201 & 0.0 & $0 \%$ \\
\hline & t1_i3 & 3 & 0 & 0.0 & 0.0 & 66 & 0.02361 & 226 & 0.0 & $100.00 \%$ \\
\hline & t1_i4 & 6 & 0.2 & 0.0 & 0.0 & 23 & 0.02 & 91 & 0.0 & $100.00 \%$ \\
\hline & t1_i5 & 0.22238 & 0.22128 & 0.01 & 0.0 & 0.02189 & 0.01776 & 177 & 0.0 & $100.00 \%$ \\
\hline & t2_i1 & & 0.047 & 0.0 & & $0 .($ & 0.0 & 158 & 0.0 & $100.00 \%$ \\
\hline & t2_i2 & 0.07670 & 0.07601 & 0.01317 & 0.00 & 0.02586 & 0.01249 & 0.00241 & 0.0 & $100.00 \%$ \\
\hline & t2_i3 & 0.13732 & 0.13490 & 0.02128 & 0.00241 & 0.04768 & 0.02071 & 0.00401 & 0.02697 & $100.00 \%$ \\
\hline & t2_i4 & 0.05997 & 0.05866 & 0.00950 & 0.00131 & 0.02139 & 0.00928 & 0.00179 & 0.01210 & $100.00 \%$ \\
\hline & t2_i5 & 0.00691 & 0.00631 & 0.00788 & 0.00060 & 0.00758 & 0.00733 & 0.00127 & 0.00026 & $17.40 \%$ \\
\hline \multirow{5}{*}{$\begin{array}{c}\overrightarrow{0} \\
\dot{0} \\
1 \\
\dot{d} \\
\dot{0}\end{array}$} & i1 & & & & & & & & & $6.80^{\circ}$ \\
\hline & i2 & & & & & & & & -0. & $30.00 \%$ \\
\hline & i3 & 5 & & & & 99 & & 67 & 0.0 & $66.80 \%$ \\
\hline & i4 & 0.02354 & 0.02350 & 0.01237 & 0.00003 & 0.01512 & 0.01219 & 0.00135 & 0.00294 & $46.80 \%$ \\
\hline & $\mathrm{i} 5$ & 0.01786 & 0.01807 & 0.00949 & -0.00021 & 0.00974 & 0.00901 & 0.00112 & 0.00073 & $50.20 \%$ \\
\hline \multirow{5}{*}{ 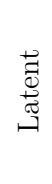 } & t1 $\xi$ & & & & & & & & & $.00 .00 \%$ \\
\hline & t2 $\xi$ & -0.05996 & -0.06054 & 0.09039 & 0.00058 & 0.06904 & 0.08633 & 0.02898 & -0.01729 & $5.60 \%$ \\
\hline & $\mathrm{t} 1 \Phi$ & 0.19298 & 0.193 & 0.02353 & -0.00004 & 0.02416 & 0.02280 & 0.00282 & 0.00136 & $100.00 \%$ \\
\hline & $\mathrm{t} 2 \Phi$ & & & 0.01449 & & 0.056 & 0.01369 & 0.00281 & 0.04308 & $100.00 \%$ \\
\hline & $\mathrm{r}$ & 0.01396 & 0.01356 & 0.01470 & 0.00041 & 0.01422 & 0.01420 & 0.00251 & 0.00002 & $17.40 \%$ \\
\hline
\end{tabular}


scale was fitted, and CFI, RMSEA, and SRMR computed. In these simulations, the fit indices should accept the model, and have only a nominal Type I error.

Figure 4 shows the average fit indices for all three scales. The average CFI increased slightly and the average RMSEA decreased slightly with increasing sample size. More importantly, already with the smallest sample size, both the average CFI and the average RMSEA correctly accepted the models, and were only marginally influenced by the sample size. However, the results were fundamentally different for the SRMR. The average SRMR exceeded its cut-off already with the smallest sample sizes; only with the larger sample sizes the average SRMR began to correctly accept the models.

Figure 5, Figure 6, and Figure 7] complement Figure 4, by showing the percentage of correctly accepted models by the CFI, RMSEA, and SRMR with their usual cut-offs, respectively. The Type I error of the RMSEA seemed the least affected by the sample size. The CFI showed an increase in the percentage of correctly accepted models, while still being adequately high even with the smallest sample size. In sharp contrast, the percentage of correctly accepted models by the SRMR was heavily affected by the sample size: with the lowest sample size, the SRMR almost never $(0 \%-1 \%)$ fell below its cut-off of .08.

To summarize, the SRMR was heavily biased in evaluating the model fits by the sample size. This effect of the sample size yielded Type I error rates of up to $100 \%$, consequently invalidating the use of the SRMR for Study 3.

\subsection{Discussion}

Study 3 suggests that applicants consistently increase their scores on personality scale items to present themselves more favourably than they normally would. This was done primarily by increasing responses to selected items. If this was done homogeneously for all items, strong measurement invariance was preserved, and an increase in the latent mean resulted (cf. preventive health behaviour in response to warning signals). If this was done homogeneously on all but a few items, strong measurement invariance was partially given, and additionally an increase in the latent mean resulted (cf. self-confidence in test situation). Lastly, if this was done heterogeneously for nearly all items, strong measurement invariance was not given, and the latent means did not differ (cf. activity in familiar communicative situation). In summary, applicants' will increase their scores on selected items, and the extent thereof may vary from item to item. The more homogeneously this is done, the more this increase will not be distinguishable from a true difference. 


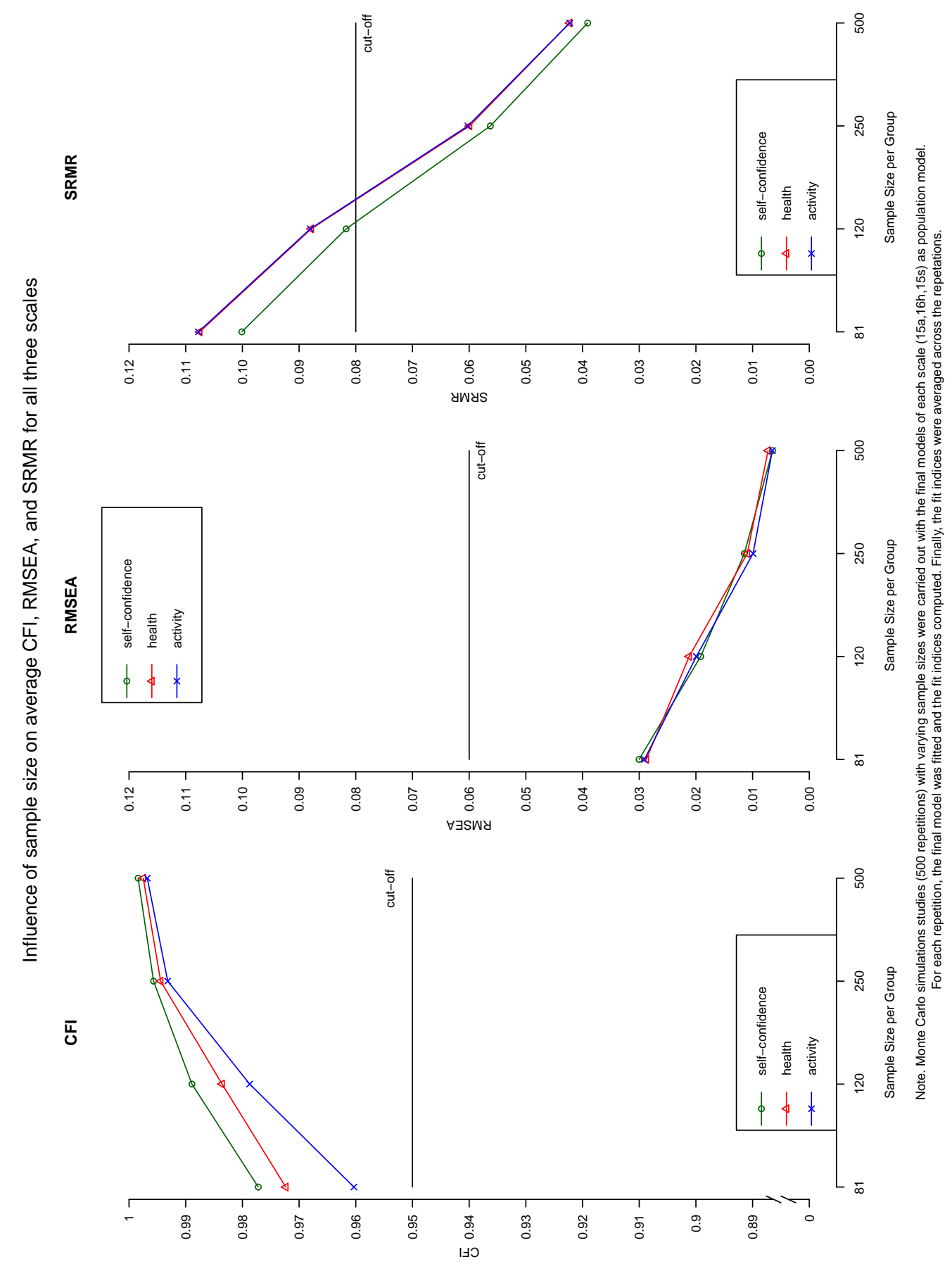

Figure 4: Comparison of the influence of sample size per group on the average CFI, RMSEA, and SRMR. 


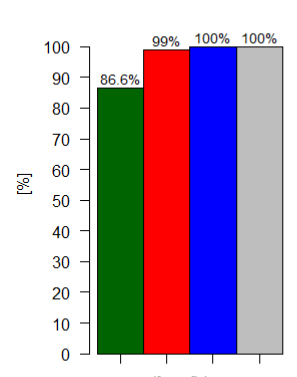

self-confidence

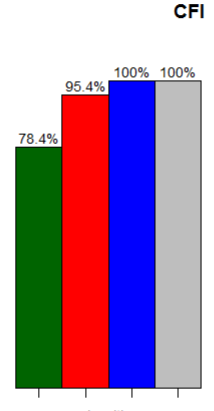

health

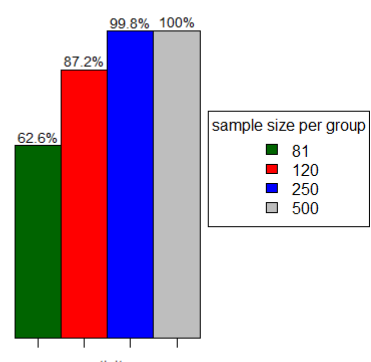

activity

Figure 5: Percentage of correctly accepted models by $C F I \geq .95$, depending on varying sample sizes.

RMSEA
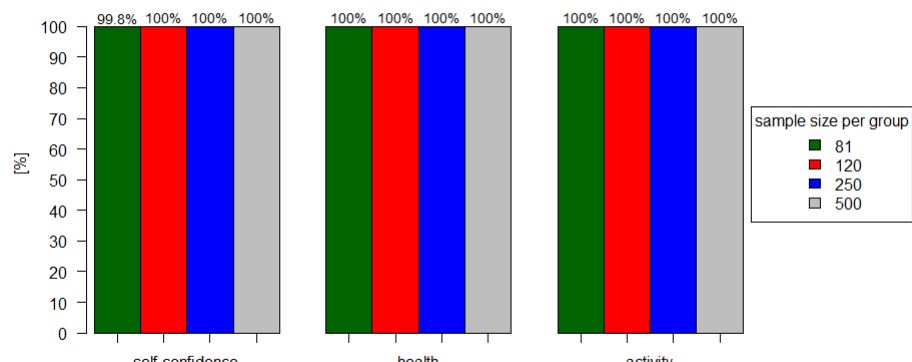

self-confidence

health

activity

Scale

Figure 6: Percentage of correctly accepted models by $R M S E A<.06$, depending on varying sample sizes.
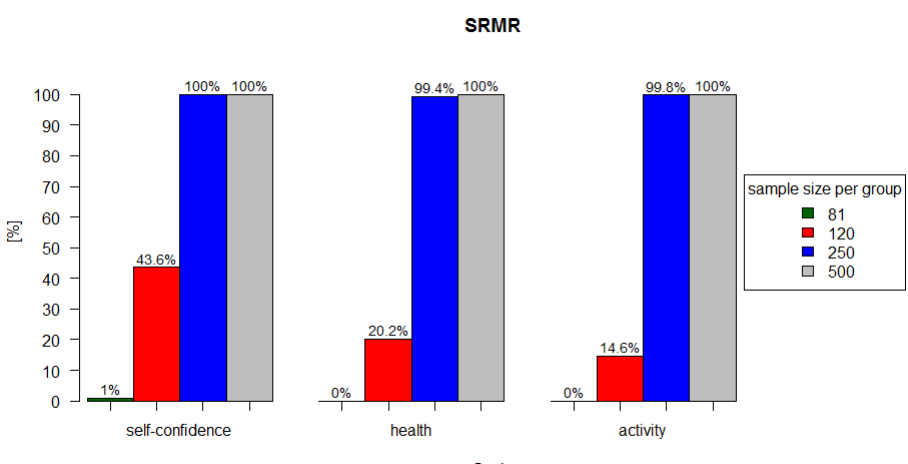

Figure 7: Percentage of correctly accepted models by $S R M R<.08$, depending on varying sample sizes. 
By contrast, the more heterogeneously this is done, the more measurement bias will be introduced.

Applicants' faking behaviour consistently affected at least some psychometric properties of the personality scales. This was contrary to the hypothesis that strict measurement invariance should hold across applicants and incumbents in the honest-condition (cf. Robie et al., 2001). On the one hand, this hypothesis was based on a single study, as there is a paucity of studies examining measurement invariance across applicants and incumbents. On the other hand, there was a key difference between Robie et al. (2001) and the current study. Robie et al. presented a warning in their study, that "distorted self-descriptions would invalidate the ... test results" (p. 195). In the current study, no such warning was presented. This may have caused differences in applicants' willingness to fake between the two studies. Prior research indicates that applicants wrongly fear that their faking behaviour may be exposable (König et al., 2012). Furthermore, studies have shown that such warnings can reduce faking when negative consequences of being exposed are emphasized (Dwight \& Donovan, 2003; Goffin \& Woods, 1995).

The violation of strong measurement invariance in two out of three scales cautions against interpreting raw mean differences of applicants and incumbents at face value (cf. Chen, 2008; Cheung \& Rensvold, 2002; Li \& Zumbo, 2009: Millsap, 2011). For preventive health behaviour in response to warning signals strong measurement invariance was given. Thus, the latent mean difference between applicants and incumbents corroborates the meta-analytic results derived from raw mean differences (cf. Birkeland et al., 2006). For the other two scales, however, the raw mean differences can be at least partially attributed to uniform measurement bias. Consequently, the raw mean score comparisons of e.g. Birkeland et al. (2006) or Study 2 (cf. Krammer \& Pflanzl, 2015) should be questioned.

However, the results of Study 3 support that the detrimental effects of applicants' faking behaviour should not be overestimated (cf. Bradley \& Hauenstein, 2006; Hogan et al., 2007; Ones et al., 2007). While applicants' faking behaviour consistently affected measurement properties, some measurement properties were also consistently preserved. The consistent preservation of weak measurement invariance implies that validity coefficients from samples of incumbents may be applicable for applicants (cf. Chen, 2008). These findings support studies showing comparable predictive validity coefficients across honest responses and selection settings (for an overview cf. Ones et al., 2007).

Finally, applicants' faking behaviour distorted the rank-orders to a modest extent. While this distortion was in line with prior studies (cf. Griffin \& Wilson, 2012; Griffith et al., 2007; Peterson et al., 2011), it corroborates 
that applicants' faking behaviour is an individual differences construct (cf. Goffin \& Boyd, 2009; Marcus, 2009; McFarland \& Ryan, 2000, 2006; MuellerHanson et al., 2006; Snell et al., 1999). Therefore, the effects of applicants' faking behaviour on selection decisions should not be taken too lightly.

\section{General Discussion}

The aim of this publication-based dissertation was to address three questions, which should be addressed for college admission processes in specific academic disciplines:

1. Should well established determinants of academic achievement be used, such as general mental ability and personality traits?

2. If personality traits are used, do applicants fake?

3. If applicants fake, does applicants' faking behaviour detrimentally affect the psychometric properties of the personality measures?

These three questions were addressed in three subsequent studies.

Study 1 (cf. Krammer et al., 2016) addressed the modelling of academic achievement. Studies have suggested that it may be useful to differentiate academic achievement measured by GPAs according to different courses types (e.g. D'Agostino \& Powers, 2009; Gustafsson \& Balke, 1993; Reeve, 2004). Study 1 showed that such a differentiation may prove especially useful for academic fields for which the education is of a highly applied nature, i.e. teacher education. This differentiation emphasizes the importance of taking into account if there are elements unique to the education in an academic field. If there are, aggregating GPAs and modelling of academic achievement should reflect such uniquenesses.

Furthermore, Study 1 examined the predictive utilities of cognitive and non-cognitive determinants of academic achievement. In line with previous studies (e.g. Credé \& Kuncel, 2008; Hell et al., 2007; Kuncel et al., 2001, 2004, 2010; Kuncel \& Hezlett, 2007), the predictive utility of general mental ability could be shown for academic achievement in teacher education. Also in line with previous studies (e.g. Farsides \& Woodfield, 2003; Poropat, 2009), non-cognitive determinants exhibited predictive utility over and above general mental ability. This importance of non-cognitive determinants suggests that in academic fields where the education is of an applied nature, determinants of prospective occupational success can already play an important role in the education. Consequently, it is worth considering not to base selection decisions for educations to these academic fields only on 
determinants of academic achievement, but additionally on determinants of occupational success.

With Study 1 corroborating the predictive utilities of non-cognitive determinants - i.e. personality traits - of academic achievement, Study 2 (cf. Krammer \& Pflanzl, 2015) examined the prevalence of applicants' faking behaviour on personality scales used for selection decisions. While respondents are highly able to distort their scores on personality inventories (cf. Viswesvaran \& Ones, 1999), applicants do so only to a negligible to small degree (cf. Birkeland et al., 2006). Furthermore, scholars have pointed out that the detrimental effects of applicants' faking behaviour may be negligible (e.g. Bradley \& Hauenstein, 2006; Hogan et al., 2007; Ones et al., 2007). In contrast, Study 2 showed that applicants increased their mean raw scores in nearly all examined personality scales. Moreover, applicants' faking behaviour affected the rank orders of applicants in line with previous studies (cf. Mueller-Hanson et al., 2003, Rosse et al., 1998; Winkelspecht et al., 2006). In addition to showing the prevalence of applicants' faking behaviour and its effects, Study 2 also underlined that applicants' faking behaviour varied between applicants and scales. These differences between applicants and scales pose a challenge for the fairness of selection decisions.

Based on the prevalence of faking on personality scales of Study 2, Study 3 (cf. Krammer et al., in press) went beyond the manifest means and correlations of Study 2, and examined the psychometric effects of applicants' faking behaviour. This was done by examining the measurement invariance across respondents responding to the same personality scales twice, once while still being applicants and later as incumbents. Such a within-subject design with a real life selection setting has been argued to constitute a golden standard in faking research (cf. Komar, Brown, Komar, \& Robie, 2008; Ryan \& Boyce, 2006: Peterson et al., 2011). Unfortunately, there is a paucity of studies combining such a within-subject design with the rigor of measurement invariance analyses.

Study 3 showed that applicants' faking behaviour consistently affected at least some of the psychometric properties of the scrutinized personality scales. The psychometric properties were mainly affected due to applicants altering their baseline probabilities of endorsing specific response categories of items. The more heterogeneously applicants increased these baseline probabilities across items of a scale, the more severe measurement properties were detrimentally affected. By contrast, applicants could also present themselves as an idealized version of themselves, by increased their baseline probabilities across items of a scale homogeneously. These results caution against treating applicants' faking behaviour as a one-size-fits-all process. Therefore, future studies should try to shed more light on the process of applicants' 
faking behaviour. Understanding the process of applicants' faking behaviour is especially important in the light of the fairness of subsequent selection decisions.

Remedying the consistently affected psychometric properties of the personality scales in Study 3, Study 3 also showed that some psychometric properties were consistently preserved. Most importantly, configural and weak measurement invariance were preserved. Weak measurement invariance is needed to compare correlations within one response condition to correlations within another response condition (Chen, 2008). Therefore, the lack of an effect on weak measurement invariance suggests that the predictive utilities of the personality scales may be preserved. This preservation of the predictive utilities is in line with previous studies, and has been used as the basis for arguing that applicants' faking behaviour may be deemed negligible in real life selection settings (for an overview cf. Ones et al., 2007).

In conclusion, yes, personality measures can and should be used for selection decisions. However, applicants' faking behaviour should not be neglected, and the process further studied. While the detrimental effects of applicants' faking behaviour seem to be limited, applicants' faking behaviour may nevertheless vary between measures and applicants. 


\section{$6 \quad$ Full Texts \& Dissemination}

Three publications formed the basis of this publication-based dissertation. These three publications are:

- Krammer, G., Sommer, M., \& Arendasy, M. E. (2016). Realistic job expectations predict academic achievement. Learning and Individual Differences, 51, 341-348. doi: 10.1016/j.lindif.2016.09.010.

- Krammer, G., \& Pflanzl, B. (2015). Faking von Persönlichkeitseigenschaften bei Zulassungsverfahren für Lehramtsstudien [Faking of Personality Measures for College Admission in Teacher Education]. Zeitschrift für Pädagogische Psychologie, 29, 205-214. doi: 10.1024/10100652/a000161.

- Krammer, G., Sommer, M., \& Arendasy, M. E. (in press). The psychometric costs of applicants' faking: examining measurement invariance and re-test correlations across response conditions. Journal of Personality Assessment. doi: 10.1080/00223891.2017.1285781

The full texts of these three publication can be found in Section 6.1. Section 6.2, and Section 6.3, respectively. Furthermore, these publications have been the basis for several talks given at national and international conferences. Sorted from oldest to most recent, these talks are:

- Weitlaner, R., \& Krammer, G. (2014). Das Zulassungsverfahren der Pädagogischen Hochschule Steiermark und dessen Validität [The college admission process of the University College of Teacher Education Styria and its predictive utility]. Paper presented at the BIMM-conference „Diversitätskompetenz in Bildungseinrichtungen“ in Salzburg, AUT, October 2014.

- Krammer, G. (2014). General mental ability over and above domainspecific measures: predicting academic achievement in a 3 year longitudinal study on college admission. Paper presented at the $15^{\text {th }}$ conference of the "International Society for Intelligence Research (ISIR)" in Graz, AUT, December 2014.

- Krammer, G., \& Pflanzl, B. (2015). Lehramtsstudienwerber/innen faken für ihren Studienplatz weniger als sie es könnten [Applicants to teacher education fake more than they could]. Paper presented at the conference of the "Österreichischen Gesellschaft für Forschung und Entwicklung im Bildungswesen (ÖFEB)" in Klagenfurt, AUT, August 2015 . 
- Krammer, G., \& Pflanzl, B. (2015). Studienerfolg in Lehramtsstudien: Eine 3-jährige Längsschnittstudie zu Intelligenz, Gesundheitsund Erholungsverhalten und realistischen Berufserwartung [Academic achievement in teacher education: a 3 year longitudinal study on intelligence, recreational and health behavior, and realistic job expectations]. Paper presented at the $80^{\text {th }}$ conference of the "Arbeitsgruppe für Empirische Pädagogische Forschung (AEPF)" in Göttingen, GER, September 2015.

- Weitlaner, R., \& Krammer, G. (2016). Competence-based admission to teacher education. Paper presented at the ENTEP-Conference "Teacher Education in Europe" in Graz, AUT, March 2016.

- Krammer, G., Sommer, M., Feldhammer, M., \& Arendasy, M. E. (2016). Der Faking-Prozess realer Bewerbungssituationen [The faking process of real-life selection settings]. Paper presented at the $11^{\text {th }}$ conference of the "Österreichischen Gesellschaft für Psychologie (ÖGP)" in Innsbruck, AUT, April 2016.

- Krammer, G., Pflanzl, B., Sommer, M. \& Arendasy, M. E. (2016). Competence-based admission to teacher education: the predictive utility of realistic job expectations. Paper presented at the $31^{\text {th }}$ "International Congress of Psychology (ICP)" in Yokohama, JP, July 2016. 
6.1 Study 1: Realistic Job Expectations Predict Academic Achievement 


\begin{tabular}{cc} 
Contents lists available at ScienceDirect \\
ELSEVIER & Learning and Individual Differences \\
\hline
\end{tabular}

\section{Realistic job expectations predict academic achievement}

Georg Krammer ${ }^{\mathrm{a}, \mathrm{b}, *}$, Markus Sommer ${ }^{\mathrm{b}}$, Martin E. Arendasy ${ }^{\mathrm{b}}$

aniversity College of Teacher Education Styria, Austria

${ }^{b}$ University of Graz, Austria

A R T I C L E I N F O

Article history:

Received 25 August 2015

2016

Accepted 16 September 2016

\section{Keywords:}

Realistic job expectation

General mental ability

Predictive utility
Teacher educatio

Academic achievement

\begin{abstract}
A B S T R A C T
Individual differences in realistic job expectations - the degree to which realistic expectations about prospective education and job are held - were introduced as a potential predictor of academic achievement. Students in teacher education $(N=176)$ completed a test battery for college admission consisting of general mental ability, reactions to stress and coping with stress, and a structured interview assessing individual differences in realistic job expectations. Grade point averages of the entire three-year long bachelor's degree and students' bachelor thesis grades served as criterion variables. Grade point averages were calculated separately for courses fostering declarative and procedural knowledge. Declarative knowledge and students' bachelor thesis grades were predicted by individual differences in general mental ability, and realistic job expectations. Contrary, procedural knowledge was predicted by individual differences in reactions to stress and coping with stress, and realistic job expectations. The current study is the first to show this predictive utility of realistic job expectations.

2016 Elsevier Inc. All rights reserved.
\end{abstract}

\section{Introduction}

Meta-analyses have already identified several cognitive determinants of academic achievement, such as general mental ability ( $g$ : e.g. Hell, Trapmann, \& Schuler, 2007; Kuncel, Hezlett, \& Ones, 2001, 2004; Kuncel, Wee, Serafin, \& Hezlett, 2010) and several non-cognitive determinants of academic achievement, for example personality traits (e.g. Poropat, 2009; O'Connor \& Paunonen, 2007; Richardson, Abraham, \& Bond, 2012). Yet, still a large portion of the variance in academic achievement remains unexplained. Therefore, there has been renewed interest in identifying determinants that may explain variance in academic achievement over and above well-established determinants (cf. Ackerman, Chamorro-Premuzic, \& Furnham, 2011; Credé \& Kuncel, 2008; Kuncel \& Hezlett, 2007; Poropat, 2009; Richardson et al., 2012). In the present article we examine the predictive utility of a new potential determinant of academic achievement: the degree to which students hold realistic expectations about their prospective education and occupation.

\subsection{Defining and measuring academic achievement}

Grade point averages (GPA) are often considered as the golden standard for measuring academic achievement. Thus, most meta-analyses (e.g. Hell et al., 2007: Kuncel et al, 2001, 2004; Poropat, 2009; Richardson et al., 2012; Trapmann, Hell, Weigand, \& Schuler, 2007) resort to GPAs to measure academic achievement. Furthermore, GPAs

* Corresponding author at: University College of Teacher Education Styria, Hasnerplatz $12,8010 \mathrm{Graz}$, Austria.

E-mail address: georg.krammer@phst.at (G. Krammer). have been shown to predict occupational status (e.g. Strenze, 2007), salary level (e.g. Roth \& Clarke, 1998), and job performance (e.g. D'Agostino \& Powers, 2009; Roth, Be Vier, Switzer, \& Schippman, 1996). In addition, studies suggest GPAs to be sufficiently reliable $($ ICC $=0.94$ : Bacon $\&$ Bean, 2006 $)$ and stable over time $\left(r_{t t}=0.83\right.$ : Kuncel et al., 2004).

Several authors (e.g. Ackerman et al., 2011; Reeve, 2004; Reeve \& Bonaccio 2011) already noted that sometimes less effort is invested in defining and measuring criterion variables than in defining and measuring predictor variables. For example, GPAs seem to be the predominant criterion variable for academic achievement. Nevertheless, GPAs are not without shortcoming. It has been shown that GPAs obtained from later on in the education do not reflect differences between students as well to individual differences in course choices and/or grading standards across and within institutions, which can lead to an underestimation of predictive validity of determinants of academic achievement (e.g. Ackerman et al., 2011; Berry \& Sackett, 2009; Didier, Kreiter, Buri, \& Solow, 2006; Elliot \& Strenta, 1988; Reeve \& Bonaccio, 2011). When considering GPAs, it therefore seems appropriate to examine GPAs of different academic years separately.

Furthermore, GPAs can be differentiated according to different courses types, e.g. courses for general knowledge, domain-specific knowledge, or applied courses (cf. D'Agostino \& Powers, 2009; Gustafsson \& Balke, 1993). There is evidence that GPAs obtained from different course types exhibit differential structural relations to determinants of academic achievement and occupational performance (cf. D'Agostino \& Powers, 2009; Gustafsson \& Balke, 1993; Reeve, 2004). Similar results have been obtained for predicting apprentices' training success, where declarative and procedural knowledge have been 
shown to exhibit differential structural relations to cognitive determinants of academic success (e.g. Häusler \& Sommer, 2006 Schmidt-Atzert, Deter, \& Jaeckel, 2004). Distinguishing between declarative and procedural knowledge may be especially relevant when considering GPAs of more applied studies, such as teacher education. Teacher education world-wide involves student-teaching (DarlingHammond \& Lieberman, 2012), and GPAs of student-teaching courses can be seen as an instance of procedural knowledge (the course requirements are similar to future occupational performance: Kuncel et al., 2004). Furthermore, grades in student-teaching courses have a higher predictive utility for future teacher performance than grades of other courses (D'Agostino \& Powers, 2009).

In addition to distinguishing between declarative and procedural knowledge, students' bachelor thesis grade could also serve as an additional differentiation of grades. Usually, every student has their own bachelor thesis topic, and a bachelor thesis needs to meet scientific quality standards. This sets bachelor thesis grades apart from course grades, as students need to transfer previously acquired knowledge, need to acquire new knowledge on their bachelor thesis topic, and have to do so autonomously. Additionally, the bachelor thesis constitutes the final and necessary step for degree completion, and degree completion has been treated as an additional measure of academic success (cf. Kuncel \& Hezlett, 2007).

\subsection{Cognitive determinants of academic achievement}

$g$ is one of the most important determinants of academic achievement (e.g. Credé \& Kuncel, 2008; Hell et al., 2007; Kuncel \& Hezlett, 2007; Kuncel et al., 2001, 2004; Kuncel et al., 2010). Although its predictive utility seems to be highest for academic achievement measured after the first academic year $(0.34 \leq \rho \leq 0.45$ : Kuncel \& Hezlett, 2007; Kuncel et al., 2001, 2004; Kuncel et al., 2010; Reeve \& Bonaccio, 2011), $g$ continues to predict academic achievement up until the very end of tertiary education $(0.20 \leq \rho \leq 0.24$ : Kuncel \& Hezlett, 2007; Kuncel et al., 2010). Several authors (e.g. Ackerman et al., 2011; Reeve \& Bonaccio, 2011) argued that this decrease in the predictive utility of $g$ may be attributable to an increase in individual differences in course choices and differential grading standards over the academic years. Thus, grades obtained later on in tertiary education are more confounded by construct-irrelevant variance, which subsequently decreases the predictive utility of $g$.

\subsection{Non-cognitive determinants of academic achievement}

Studies (e.g. Ackerman \& Heggestad, 1997; Farsides \& Woodfield, 2003; O'Connor \& Paunonen, 2007; Paunonen \& Ashton, 2001; Poropat, 2009) indicate that personality traits predict academic achievement. Conscientiousness $(\rho=0.22)$, openness $(\rho=0.12)$, and agreeableness ( $\rho=0.07$ ) have been demonstrated to be such non-cognitive determinants of academic achievement (Poropat, 2009). While the predictive utility of personality traits is smaller than that of $g$, it prevails even when accounting for $g$ (e.g. Farsides \& Woodfield, 2003; Poropat, 2009).

Facets of conscientiousness and openness even outperform their respective Big Five factor domains in predicting academic achievement (cf. O'Connor \& Paunonen, 2007; Paunonen \& Ashton, 2001). This indicates that facets of the Big Five domains may be differentially linked to academic achievement. This may also be the case for emotional stability, academic achievement. This may also be the case for emotional stability, (cf. Ackerman \& Heggestad, 1997; Farsides \& Woodfield, 2003), while failing to do so ( $\rho=0.02$ : Poropat, 2009). Emotional stability comprises several facets such as anxiety, angry hostility, depression, self-consciousness, impulsiveness, and vulnerability or stress tolerance (for an sciousness, impulsiveness, and vulnerability or stress tolerance (for an overview: Arendasy, Sommer, \& Feldhammer, 2011; Costa \& McCrea, test anxiety: $-0.21 \leq \rho \leq-0.33$; reactions to stress:
$-0.11 \leq \rho \leq-0.14$; Ackerman \& Heggestad, 1997; Hembree, 1988 . Richardson et al., 2012), while others are not (e.g. depression: $\rho=-0.03$ : Richardson et al., 2012). These different predictive utilities of emotional stability facets may explain why studies failed to support the predictive utility of emotional stability.

Consequently, more specific facets of emotional stability may be useful for predicting academic achievement For instance, the emotional stability facet reaction to stress (cf. Ackerman \& Heggestad, 1997) may be a determinant of success in the teaching profession, where stress and burnout are prevalent (e.g. Schaarschmidt, 2005; Schaarschmidt \& Kieschke, 2007). Indeed, teachers' stress has been linked to important student behaviours, such as not paying attention in class $(r=0.39)$ and hostility toward the teacher ( $r=0.40$ : Geving 2007). Furthermore, strategies for coping with stress have been shown to be related to grades in student-teaching courses (Rieder, 2011). Unfortunately, there are currently no studies examining the predictive utility of selected emotional stability facets for predicting more conventional criteria of academic success, such as GPAs.

1.4. Realistic job expectations

The accuracy of students' expectations of their educational and professional career may be another important determinant of academic success. Students do not always hold realistic expectations of studying at university, and providing them with more realistic information on what to expect reduces attrition (e. Braxton, Vesper, \& Hossler, 1995; Cooke, Sims, \& Peyrefitte, 1995; Earnest \& Dwyer, 2010). Provid1995; Cooke, Sims, \& Peyrefitte, 1995; Earnest \& Dwyer, 2010). Provid-
ing students with such realistic information lowers the initial expectations, and increases self-selection, organizational commitment, satisfaction, and possibly even academic achievement (e.g. Braxton et al., 1995; Cooke et al., 1995; Earnest \& Dwyer, 2010). Actually, this strategy was adopted from a recruitment method used by organizations: realistic job previews (cf. Phillips, 1998; Premack \& Wanous, 1985; Rynes, 1991). Providing realistic job previews influences how initial expectations are met, which in turn influences job satisfaction, organizational commitment, the intent do leave, job survival, and moderately job performance (e.g. Wanous, Poland, Premack, \& Davis, 1992). In the same way, when students have more accurate expectations, it should lead to having better met expectation, which in turn may affect academic achievement. Unfortunately, most studies so far conducted in the academic context used an intervention design and solely focused on increasing the accuracy of students' expectations regarding more general aspects of studying at university. It therefore might be useful to evaluate, whether individual differences in students' expectations about their prospective educational and occupational career - realistic job expectations (RJE) - have predictive utility in explaining individual differences in academic achievement.

1.5. Formulation of the problem

In the present study, the predictive utility of RJE for academic achievement was examined in the context of teacher education. This specific domain was chosen due to the applied nature of its curriculum, which allows differentiating academic achievement into declarative and procedural knowledge. In addition, there are renewed efforts to improve teacher education in Austria and interest in selecting successful teachers rose in the last few years (Mayr, 2012). The next section outlines the research hypotheses with regard to the measurement of the criterion variable academic achievement and the predictive utilities of the determinants.

1.5.1. Hypotheses regarding the measurement of academic achievement Prior research (e.g. Reeve \& Bonaccio, 2011) suggested that grades have a higher saturation of construct-relevant variance in the first years of education than later on. This should affect factor loadings of academic achievement indicators. More precisely, GPAs obtained later on 
in the education should have significantly lower factor loadings than GPAs earlier on. To our knowledge, this hypothesis has not yet been empirically tested, especially not considering declarative and procedural facets of academic achievement. Testing this hypothesis will contribute to our understanding of the degree to which GPAs of different academic years actually measure academic achievement alike.

1.5.2. Hypotheses regarding the predictive utility of general mental ability

Prior research has shown that $g$ predicts academic achievement (Credé \& Kuncel, 2008; Hell et al., 2007; Kuncel \& Hezlett, 2007; Kuncel et al., 2001, 2004; Kuncel et al., 2010). $g$ has shown predictive utility for declarative and procedural knowledge, while it may be less relevant for procedural than for declarative knowledge (cf. Häusler \& Sommer, 2006; Schmidt-Atzert et al., 2004). This might be particularly true for teacher education, where Kuncel et al. (2004) reported that $g$ does not predict procedural knowledge $(\rho=-0.04)$. The authors dismissed their finding, explaining their null-result with sampling error and problems associated with measuring their criterion variable. Alternatively, their null-finding might reflect that $g$ possesses less predictive utility for procedural knowledge in this particular domain at a certain level of expertise (for a similar argument: Ackerman, 2003). Therefore, we hypothesized that $g$ has a higher predictive utility for declarative than procedural knowledge. Furthermore, $g$ should also predict students' bachelor thesis grades, as knowledge acquisition is vital for the bachelor thesis, and in turn $g$ should have predictive utility.

1.5.3. Hypotheses regarding the predictive utility of non-cognitive determinants

Studies show mixed results on the predictive utility of emotional stability for academic achievement (cf. Ackerman \& Heggestad, 1997; Farsides \& Woodfield, 2003; Poropat, 2009). One reason for the inconsistent findings may be that some emotional stability facets predict academic achievement, while others do not (cf. Ackerman \& Heggestad, 1997; Hembree, 1988; Richardson et al., 2012). We therefore hypothesized that the emotional stability facet coping with stress and reaction to stress (cf. Ackerman \& Heggestad, 1997; Geving, 2007; Rieder, 2011 Richardson et al., 2012) should contribute to the prediction of academic achievement even when controlling for $g$. Furthermore, comparing validity coefficients across studies revealed higher coefficients for procedural knowledge than for declarative knowledge (cf. Ackerman \& Heggestad, 1997; Geving, 2007; Richardson et al., 2012; Rieder, 2011). Therefore, coping with stress and reaction to stress (CRS) should have a higher predictive utility for procedural knowledge than for declarative knowledge and students' bachelor thesis grades.

In this study, RJE are introduced as a potential determinant of academic achievement. It has not yet been examined whether individual differences in RJE contribute to the prediction of academic achievement. The present study therefore examines, whether RJE can account for individual differences in declarative knowledge, procedural knowledge, and students' bachelor thesis grades.

\section{Method}

2.1. Measures

2.1.1. Coping with stress and reaction to stress

CRS was measured by means of the domain factor scale health and recreational behavior of the Inventory for Personality Assessment in Situations (IPS: Schaarschmidt \& Fischer, 2013). The IPS contains three subscales assessing the higher order factor health and recreational behavior: relaxation after a working day (RE), active recovery behavior in leisure (RB), and health prevention due to warning signals (HP). The IPS is a German personality inventory that has been specifically developed for professions with high psychosocial demands such as the teacher profession. These scales possess face-validity, as their content is evidently important for teacher education (e.g. Krammer \& Pflanzl, 2015b).
Furthermore, several validation studies confirmed that the scales of this inventory measure personality dispositions relevant to the teaching profession, and that teachers and students in teacher education exhibit characteristic personality profiles that are similar across these two groups but differ markedly from students of other disciplines (cf. Mayr \& Brandstätter, 1998; Schaarschmidt \& Fischer, 2013).

For each scale, a situational context is given. E.g. for health prevention due to warning signals the situational context is that you have not been feeling too well for a longer time and that people have already mentioned this to you suggesting a doctor's appointment. The items address personal behavior that may occur in the described situation (e.g. "to act cautious and preventive"). For relaxation after a working day, the situational context addresses the evening after the end of a working day, and the personal behavior how this evening is used (e.g. "to enjoy it fully"). For active recovery behavior in leisure, the situational context addresses having spare time to use, and the personal behavior how the spare time is used (e.g. "to intensely dedicate myself to leisure activities"). The task of the respondents is to indicate the extent to which they would exhibit the personal behavior in the given situation using these scales comprises five items. Schaarschmidt and Fischer (2013) report satisfactory intern consistency $(0.71 \leq \alpha \leq 0.91)$ and good re-test reliabilities $\left(0.70 \leq \mathrm{r}_{\mathrm{tt}} \leq 0.88\right)$ for these scales.

\subsubsection{Realistic job expectations}

RJE were assessed with a structured interview designed specifically for college admission in teacher education. Every applicant was interviewed by two assessors for approximately $15 \mathrm{~min}$, and rated by both assessors independently. The prerequisite for being an assessor was having a pedagogical education.

The structured interview contained eight items. Three items were general items regarding the overall poise of the applicants, i.e. an item addressed the manner of the verbal expression of the applicants. These general items were omitted for the present analysis, as they do not assess RJE. RJE were assessed with the remaining five items, each rated on a 4 point Likert-scale (definitely true to not true at all). For example, one item addressed knowledge of current pedagogical affairs, like changes in school reforms (i.e. "the applicant is knowledgeable on current pedagogical issues"), while another item addressed the professional experience of the applicants relevant for the pedagogical context (i.e. "the applicant has experienced pedagogical settings relevant for the teaching profession"). Previous research using CFA indicated that these five items of the structured interview are essentially uni-dimensional for each of the two raters (for further details: Krammer \& Pflanzl, 2015a), and exhibited sufficient intern consistencies (R1: $\alpha=0.831$; $\mathrm{R} 2: \alpha=0.850)$

\subsubsection{General mental ability}

The fluid intelligence $\left(\mathrm{G}_{\mathrm{f}}\right)$ subtests numerical-inductive reasoning (NIR) and figural-inductive reasoning (FIR) of the Intelligence Structure Battery (INSBAT: Arendasy et al., 2012) were used to measure $g$. The INSBAT is a commonly used German intelligence test battery constructed on the basis of the hierarchical intelligence model of Cattell-HornCarroll (Carroll, 1993; Horn \& Noll, 1997). Both subtests were administered as computerized adaptive tests (CAT: van der Linden \& Glas, 2000) with the target reliability set to an equivalent of Cronbach's $\alpha=0.70$. As soon as this target reliability was reached, the adaptive testing algorithm terminated.

The subtest numerical inductive reasoning consisted of number series problems. Respondents had to identify the rules governing the number series, and to complete the number series by providing the next number. This subtest was constructed by means of automatic item generation and has been shown to exhibit good psychometric properties (Arendasy \& Sommer, 2012; Arendasy et al., 2012).

The subtest figural-inductive reasoning consisted of classic $3 \times 3$ figural matrices problems. Respondents had to identify the rules governing 
the matrices and to complete each matrix with one of eight answer alternatives. This subtest was constructed on the basis of a detailed construction rationale, and has exhibited good psychometric properties (Arendasy et al., 2012; Hornke, 2002; Hornke, Etzel, \& Küppers, 2000).

\subsubsection{Academic achievement}

For academic achievement, all grades of an entire bachelor's degree of education were used. Within the curricula of the University College of Teacher Education Styria, the courses can be classified into four major domains: a) social sciences, b) subject matter and teaching methodology, c) complimentary studies, and d) student-teaching. These four domains can be classified into two even broader domains: courses where students study ( $\mathrm{a}, \mathrm{b}$, and c), and courses where students teach (d). Former conveys declarative knowledge, latter conveys procedural knowledge. For the latter, the students were assigned to schools where they regularly had to hold classes, along with the necessary preparation and post-processing, supervision, and mentoring. Student-teaching is part of the curriculum in each semester and makes up one fifth of the curriculum. Additionally, we obtained students' bachelor thesis grade as a final measure of academic achievement. In summary, the grades separated for declarative and procedural knowledge, and the grade of the bachelor thesis were used as criteria.

\subsection{Sample}

In the academic year 2011/12, 237 applicants were accepted and began to study at the University College of Teacher Education Styria. Of these 237 students, 176 finished their bachelor's degree three years later. These 176 graduates comprised the final sample. Thereof, 144 (82\%) were female and 32 (18\%) male (ratio within the 237 applicants: female/male $74 \% / 26 \%$ ), and 112 (64\%) were training to become teachers in primary and $64(36 \%)$ in secondary education (ratio within the 237 applicants: primary/secondary $~ 57 \% / 43 \%$ ). At the beginning of the academic year $2011 / 12$, the sample was between 20.5 and 52.6 years old $(M=25.4, S D=5.4)$. Age did not differ between the graduates and non-graduates $(t[235]=0.351, p=0.726, d=0.05)$; neither did CRS $(t[235]=0.002, p=0.998, d=0.00)$, RJE $(t[235]=-1.797, p=0.074, d=-0.27)$, nor $g(t[235]=-0.619$, $p=0.536, d=-0.09$ ), although there was a small effect for RJE.

\subsection{Procedure}

All the data for the predictors were collected within the college admission process in the academic year 2011/12. For college admission, the applicants completed the measures for intelligence and personality, the applicants completed the measures for intelligence and personality, and finally the applicants were interviewed. The admitted students
studied for three years to obtain their bachelor's degree until the end of the academic year 2013/14. Within the last academic year of their education, students had to begin and finish their bachelor thesis for degree completion. All grades are managed electronically in one database for all students by the university administration and were culled from this students by
database.

\subsection{Tested models}

In line with current best practice standards in structural equation modeling (cf. Mueller \& Hancock, 2008) the structural relations between the latent predictor and criterion variables were evaluated in a two-step process. Firstly, the measurement models for the predictor and criterion measures were evaluated separately to rule out a potential misfit of the full structural model being attributable to a misfit in the measurement models. In a second step, the two measurement models were combined into a full structural model to examine the predictive utility of the latent predictors for the criteria.

The indicators for the measurement models of the latent predictors were 1) the three IPS-subscales (RE, RB, and HP) for CRS, 2) the mean scores of the raters of the standardized interview (R1 and R2) for the RJE, and 3 ) the two INSBAT-subscales (FIR and NIR) for $g$. The three latent factors (CRS, RJE, $g$ ) were allowed to be correlated to examine potential multicollinearity.

The measurement model of the latent criteria had two factors, one representing declarative and one procedural knowledge. Both factors were measured by GPAs from the $1^{\text {st }} 2^{\text {nd }}$ and $3^{\text {rd }}$ academic year according to curriculum. Based on previous studies (Häusler \& Sommer, 2006; Schmidt-Atzert et al., 2004) the latent declarative and procedural knowledge factors were allowed to correlate.

Factor loadings from the latent declarative and procedural knowledge factors to their respective indicators were examined to test the hypothesis on the saturation of construct-relevant variance within the GPAs. Therefore, these factor loadings were restricted to be equal within each latent knowledge factor for the academic years. If the resulting restricted model fitted the data worse than the unrestricted model (Cheung \& Rensvold, 2002), pairwise comparisons followed to determine which academic years differed.

In a final step, the two measurement models were combined into a full structural model and augmented by including the students' bachelor thesis grade as criterion variable. Direct paths lead from all three latent predictors to the correlated latent knowledge factors. In addition, direct paths lead from $g$, RJE, and declarative knowledge to the bachelor thesis grade. The direct paths from factors CRS and procedural knowledge to the thesis grade were set to zero, as writing a bachelor thesis does not involve teaching of any sort, and should also be independent of individual differences in CRS.

\subsection{Evaluation of the model fit}

The following cut-offs for the GoF indices were used to evaluate the global model fits: non-significant $\chi^{2}$ statistic, root mean square error approximation (RMSEA) $<0.08$, comparative fit index (CFI) $\geq 0.95$, and standardized root mean square residual (SRMR) $<0.08$ (cf. Hu \& Bentler, 1999; Marsh, Hau, \& Wen, 2004). For local model fit, critical ratio tests tested whether the path coefficients statistically differed from zero $(p<0.05)$. The changes in $\chi^{2}$ statistic and CFI were used to compare nested models: non-significant changes in $\chi^{2}$ statistic and $\Delta \mathrm{CFI}<0.01$ indicated equivalent model fit (Cheung \& Rensvold, 2002).

\section{Results}

3.1. Descriptive results

Table 1 provides descriptive statistics and correlations of the manifest variables. Manifest variables of the same latent factor were positively correlated, and correlations between manifest variables of different latent factors were generally smaller. Multivariate normality was given neither for the indicator variables of the predictors, the indicator variables of the criteria, nor for the all manifest variables (Mardia test: all multivariate skewness $\geq 40.05$, all $p<0.001$ ). WLSMV (cf. Lei, 2009; Savalei \& Rhemtulla, 2013) was used for parameter estimation, due to the lack of multivariate normality and that indicators of the criterion variables constituted categorical variables. All structural equation models were calculated with lavaan (Rosseel, 2012).

\subsection{Measurement models}

The measurement model of the predictor variables fitted the data well $\left(\chi^{2}[11]=17.655, p=0.09\right.$, RMSEA $<0.01$, CFI $>0.99$, SRMR $=$ 0.042 ). All factor loadings were of the expected magnitude and statistically significant. Furthermore, $g$ correlated neither with $\operatorname{RJE}(r=-0.15$, $p=0.28)$, nor with CRS $(r=0.02, p=0.86)$, and RJE and CRS were also not correlated $(r=0.15, p=0.09)$.

The measurement model of the criterion variables fitted the data well $\left(\chi^{2}[8]=6.651, p=0.575\right.$, RMSEA $<0.01$, CFI $>0.99$, SRMR $=$ 
0.029). Again, all factor loadings were statistically significant. In line with theoretical expectations, declarative and procedural knowledge were highly correlated $(r=0.92, p<0.001)$. However, the latent correlation did not reach 1 , suggesting that differentiating between declarative and procedural knowledge is feasibly despite this high correlation. Next, factor loadings of the declarative knowledge indicators were constrained to being equal. This restricted model $\left(\chi^{2}[9]=6.476, p=\right.$ 0.691 , RMSEA $<0.01$, CFI $>0.99$, SRMR $=0.033$ ) did not fit the data worse than the unrestricted model $\left(\Delta \chi^{2}[1]=0.175, p=0.676\right.$, $\Delta \mathrm{CFI}<0.001)$. However, a model with equality constraints on factor loadings of the procedural knowledge indicators did not fit the data $\left(\chi^{2}[9]=54.250, p<0.001\right.$, RMSEA $=0.170, \mathrm{CFI}=0.584$, SRMR $=$ $0.167)$ and also fitted significantly worse than the unrestricted model $\left(\Delta \chi^{2}[1]=47.599, p<0.001, \Delta \mathrm{CFI}=0.416\right)$. Thus, the hypothesis that GPAs obtained in later years are less saturated by the latent construct only held for the procedural knowledge factor. Pairwise comparisons indicated that constraining the factor loading of the $1^{\text {st }}$ and $2^{\text {nd }}$ academic year to be equivalent $\left(\chi^{2}[9]=11.385, p=0.250\right.$, RMSEA $=0.039$, $\mathrm{CFI}=0.978$, SRMR $=0.041$ ) resulted in a significantly worse model fit $\left(\Delta \chi^{2}[1]=4.734, p=0.030, \Delta \mathrm{CFI}=0.022\right)$. Similar results were obtained for the $2^{\text {nd }}$ and $3^{\text {rd }}$ academic year $\left(\chi^{2}[9]=41.966, p<0.001\right.$, RMSEA $=0.145$, CFI $=0.697$, SRMR $=0.117 ; \Delta \chi^{2}[1]=35.315$, $p<0.001, \Delta \mathrm{CFI}=0.303)$, and the $1^{\text {st }}$ and $3^{\text {rd }}$ academic year $\left(\chi^{2}[9]=\right.$ $50.499, p<0.001$, RMSEA $=0.162$, CFI $=0.619$, SRMR $=0.152$; $\left.\Delta \chi^{2}[1]=43.848, p<0.001, \Delta \mathrm{CFI}=0.381\right)$. An inspection of the standardized factor loading estimates (cf. Fig. 1) indicated that the trait saturation of the year-wise GPAs of the latent procedural knowledge factor gradually decreased from the $1^{\text {st }}$ to the $3^{\text {rd }}$ academic year.

3.3. Full structural model

Next, the fit of the full structural model was examined. This model fitted the data well $\left(\chi^{2}[68]=73.351, p=0.307\right.$, RMSEA $=0.021$, $\mathrm{CFI}=0.979$, SRMR $=0.048$ ). However, the paths from declarative knowledge to students' bachelor thesis grades $(\beta=0.066, p=$ $0.444)$, from CRS to declarative knowledge $(\beta=0.128, p=0.185)$, and from $g$ to procedural knowledge $(\beta=0.064, p=0.643)$ failed to reach significance. Since this invalidated the initial full structural model (cf. Marsh et al., 2004), a revised full structural model was calculated wherein these paths were fixed to zero. The revised full structural model fitted the data well $\left(\chi^{2}[71]=76.004, p=0.319\right.$, RMSEA $=0.020, \mathrm{CFI}=0.980$, SRMR $=0.054)$. The standardized factor loadings and regression coefficients of the revised full structural equation model are shown in Fig. 1, details for all regression coefficients in Table 2. All factor loadings and regression coefficients were statistically significant $(p<0.05)$. Given that the paths from CRS to declarative knowledge and from $g$ to procedural knowledge were essentially zero, while the paths to the respectively other knowledge domain differed significantly from zero, there was no need to further test the hypotheses regarding the differentiated predictive utility of CRS and $g$. In summary, $g$ predicted declarative knowledge and students' bachelor thesis grades, CRS the procedural knowledge, and finally RJE all three criteria.

\section{Discussion}

In the present study, individual differences in realistic job expectations (RJE) were introduced as a new determinant of academic achievement. The predictive utility of RJE over and above well-established determinants of academic achievement such as $g$ and emotional stability facets was examined and shown. In addition, it was evaluated whether the predictive utility of these determinants generalizes across procedural and declarative knowledge measures of academic achievement. 


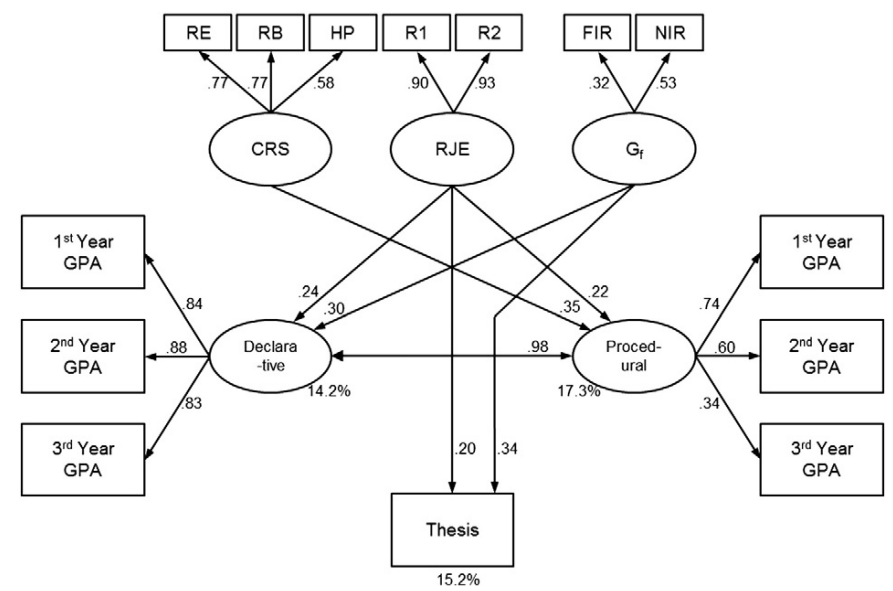
Fig. 1. The revised full structural model $\left(\chi^{2}[71]=76.004, p=0.319, \mathrm{RMSEA}=0.020, \mathrm{CFI}=0.980, \mathrm{SRMR}=0.054\right)$ with standardized path coefficients. For the declarative knowledge,
procedural knowledge, and the grade on the bachelor thesis, the amount of explained variance is given. Note: $\mathrm{CRS}=$ coping with stress and reaction to stress: RJE $=$ realistic job expectations: $G_{-}=$fluid intelligence, $\mathrm{RE}=$ relaxation after a working day: $\mathrm{RB}=$ active recovery behavior in leisure; $\mathrm{HP}=$ health prevention because of warning signals; $R 1$ and $R 2=$ $1^{\text {st }}$ and $2^{\text {nd }}$ rater of the structured interview; NIR = numerical inductive reasoning; FIR = figural inductive reasoning, GPA = grade point average.

\subsection{Measuring and modeling academic achievement}

While GPAs are one of the most commonly used measures of academic achievement (e.g. Hell et al., 2007; Kuncel et al., 2001, 2004; Poropat, 2009; Richardson et al., 2012; Trapmann et al., 2007), it has been suggested that GPAs of different academic years do not reflect academic achievement alike (e.g. Reeve \& Bonaccio, 2011). Our results corroborate this potential incomparability of GPAs only for grades of courses conveying procedural knowledge. One should therefore carefully consider from what academic year GPAs of procedural knowledge courses are obtained, as it seems that for these courses GPAs of different academic years may not reflect academic achievement alike. In contrast, we found invariance of the latent trait saturation across academic years of grades from courses conveying declarative knowledge. This invariance attenuates this potential incomparability of GPAs, however only for grades of procedural knowledge courses. These findings also demonstrate a key difference between declarative and procedural knowledge, even if both facets of academic achievement have been shown to be highly correlated as in previous studies (cf. Häusler \& Sommer, 2006; Schmidt-Atzert et al., 2004).

Table 2

Unstandardized (b) and standardized regression ( $\beta$ ) coefficients of the revised full structural model, with the amount of variance explained for the three criterion variables $\left(\mathrm{R}^{2}\right)$.

\begin{tabular}{lllllll}
\hline Criterion & Predictor & $\mathrm{b}$ & $\mathrm{SE}$ & $\beta$ & $p$ & $\mathrm{R}^{2}$ \\
\hline Declarative knowledge & $\mathrm{RJE}$ & 0.061 & 0.024 & 0.235 & 0.011 & $14.2 \%$ \\
& $\mathrm{G}_{\mathrm{f}}$ & 0.077 & 0.030 & 0.295 & 0.011 & \\
Procedural knowledge & $\mathrm{CRS}$ & - & - & - & - & \\
& $\mathrm{RJE}$ & 0.045 & 0.022 & 0.220 & 0.041 & $17.3 \%$ \\
& $\mathrm{G}_{\mathrm{f}}$ & - & - & - & - & \\
\multirow{5}{*}{ Thesis } & $\mathrm{CRS}$ & 0.072 & 0.025 & 0.353 & 0.003 & \\
& $\mathrm{RJE}$ & 0.150 & 0.070 & 0.197 & 0.033 & $15.2 \%$ \\
& $\mathrm{G}$ & 0.257 & 0.080 & 0.337 & 0.001 & \\
& $\mathrm{CRS}$ & - & - & - & - & \\
\hline
\end{tabular}

Note. $\mathrm{CRS}=$ coping with stress and reaction to stress; RJE $=$ realistic job expectations: $\mathrm{G}_{\mathrm{f}}=$ fluid intelligence; $\mathrm{SE}=$ standard error of the unstandardized regression coefficients; $p=p$-value of the unstandardized regression coefficients.

\subsection{The predictive utility of general mental ability}

$g$ constitutes one of the most important determinants of academic achievement (e.g. Credé \& Kuncel, 2008; Hell et al., 2007; Kuncel \& Hezlett, 2007; Kuncel et al., 2001, 2004; Kuncel et al., 2010). However, studies suggest that procedural knowledge facets of academic achievement are less well predicted by $g$ than declarative knowledge facets (e.g. Häusler \& Sommer, 2006; Kuncel et al., 2004; Schmidt-Atzert et al, 2004). Our results are in line with the hypotheses that $g$ predicts declarative knowledge acquisition (latent declarative knowledge factor), and transfer of acquired declarative knowledge (bachelor thesis), but does not account for procedural knowledge acquisition relevant to teacher education. This finding underlines the usefulness of distinguishing between different facets of academic achievement. Moreover, this finding advises to keep in mind that while $g$ is an important determinant of academic achievement, $g$ does not determine all facets of academic achievement alike.

\subsection{The predictive utility of emotional stability facets}

One reason for the mixed findings concerning the predictive utility of the Big Five domain factor emotional stability could be that some emotional stability facets predict academic achievement, while others do not (cf. Ackerman \& Heggestad, 1997; Farsides \& Woodfield, 2003; Hembree, 1988; Richardson et al., 2012; Poropat, 2009). We therefore examined an emotional stability facet relevant for the specific context given, and it showed predictive utility. While it was beneficial for the acquisition of procedural knowledge it was not for the acquisition of declarative knowledge or students' bachelor thesis grades. The present results are in line with the hypothesis that facet-level measures can have a higher predictive utility than domain-level measures (e.g. emotional stability: $\rho=0.02$ : Poropat, 2009). However, this finding seems to be confined to the procedural facet of academic achievement, and in turn demonstrates the importance of occupationally relevant variables for more applied study majors, such as teacher education. 
4.4. The predictive utility of realistic job expectations

RJE were introduced as a new potential determinant of academic achievement. While students benefit from interventions increasing the accuracy of their expectations of studying at university (e.g. Braxton et al., 1995; Cooke et al., 1995; Earnest \& Dwyer, 2010), no study conducted so far linked individual differences in RJE to more conventional measures of academic achievement, such as GPAs. The present study indicates that RJE have predictive utility for procedural and declarative facets of academic achievement alike. Despite its range restriction, the predictive utility of RJE was similar to the predictive utility reported for conscientiousness ( $\rho=0.22$ : Poropat, 2009). RJE also predicted students' bachelor thesis grades over and above $g$. A potential explanation of these findings might be that RJE are strongly content specific, and partly conceptually linked to domain specific interest (Krapp, 1992; cf. Krapp \& Prenzel, 2011, for an overview of domain specific interest)

\subsection{Limitations and conclusion}

Like all studies, the present one is not without limitations. Firstly, the data were obtained in the teacher education domain and therefore should not be carefreely generalized to other bachelor degree studies. Furthermore, the sample only comprised students, who graduated within the minimum amount of time. The sample therefore cannot be regarded as entirely representative. Future studies should attempt to replicate the present findings with a more heterogeneous student body and students from other domains. A cross-validation on an independent sample also seems appropriate due to post hoc modifications of the full structural equation model.

The second limitation concerns the measurement of the predictor variables. The present study was conducted in a real-life admission testing setting Therefore the predictor variables used were also used for admission testing. Although there is evidence that fluid intelligence and $g$ are nearly indistinguishable (e.g. Arendasy, Hergovich, \& Sommer, 2008; Gustafsson \& Balke, 1993), future studies may consider a broader scope of cognitive abilities. A similar argument can be made for the personality traits. A revised test battery may include measures of openness and conscientiousness, which have been shown to be important determinants of academic achievement (e.g. Farsides \& Woodfield, 2003; Poropat, 2009). Ideally, these personality traits would be measured at the domain-level and facet-level. Including domain-level and facet-level measures would allow to compare their predictive utility for different facets of academic achievement.

Despites these limitations, we believe that the present study is a first step in examining the predictive utility of RJE as a potential additional predictor of academic success. Furthermore, the present study outlines the usefulness of distinguishing between different facets of academic success. General mental ability (g), coping with stress and reactions to stress (CRS), and realistic job expectations (RJE) were differentially linked to individual differences in declarative and procedural knowledge, which corroborates and extents previous findings obtained in the field of predicting apprentices' training success (e.g. Häusler \& Sommer, 2006; Schmidt-Atzert et al., 2004). In conclusion, realistic job expectations (RJE) were not only a new potential predictor for academic achievement, but are a new predictor with potential.

\section{References}

Ackerman, P. L. (2003). Cognitive ability and non-ability trait determinants of expertise. Educational Researcher, 32, 15-20.

Ackerman, P. L., \& Heggestad, E. D. (1997). Intelligence, personality, and interests: Evidence of overlapping traits. Psychological Bulletin, 121, 219-245.

Ackerman, P. L., Chamorro-Premuzic, T., \& Furnham, A. (2011). Trait complexes and academic achievement: Old and new ways of examining personality in educational conArendasy, M., \& Sommer, M. (2012). Using automatic item generation to meet the in-
creasing item demands of high-stakes assessment. Learning and Individual Differences, 22, 112-117.

Andasy, M., Hergovich, A., \& Sommer, M. (2008). Investigating the g-saturation of various stratum-two factors using automatic item generation. Intelligence, 36, 574-583. Arendasy, M., Sommer, M., \& Feldhammer, M. (2011). Manual Big Five Structure Inventory
(BFSI). Mödling: Schuhfried.

endasy, M., Hornke, L. F., Sommer, M., Häusler, J., Wagner-Menghin, M., Gittler, G., ... Schuhfried.

Bacon, D. R., \& Bean, B. (2006). GPA in research studies: An invaluable but neglected opportunity. Journal of Marketing Education, 28, 35-42.

Pry, C. M., \& Sackett, P.R. (2009). Individual differences in course choice result in underestimation of the validity of college admissions systems. Psychological Science, 20,

Braxton, J. M., Vesper, N., \& Hossler, D. (1995). Expectations for college and student persistence. Research in Higher Education, 36, 595-612

A survey of factor-analytic studies. Cam-

bridge: Cambridge University Press.
Cheung, G. W., \& Rensvold, R. B. (2002). Evaluating goodness of fit indexes for testing measurement invariance. Structural Equation Modelling, 9, 233-255.

Cooke, D. K., Sims, R. L., \& Peyrefitte, J. (1995). The relationship between graduate student attitudes and attrition. Journal of Psychology, 129, 677-688.

Costa, P. T., \& McCrea, R. R. (1995). Domains and facets: Hierarchical personality assessment using the revised

Credé, M.., \& Kuncel, N. R. (2008). Study habits, skills, and attitudes. Perspectives on Psychological Science, 3, 425-453.

'Agostino, J. V., \& Powers, S. J. (2009). Predicting teacher performance with test scores and grade point average: A meta-analysis. American Educational Research Journal, 6, 146-182.

Darling-Hammond, L., \& Lieberman, A. (2012). Teacher education around the world: Changing policies and practices. New York: Routledge.

, Kreiter, C. D., Buri, R., \& Solow, C. (2006). Investigating the utility of a GPA institutional adjustment

Earnest, D. R., \& Dwyer, W. O. (2010). In their own words: An online strategy for increasing stress-coping skills among college freshmen. College Student Journal, 44, 888-900. liot, R., \& Strenta, A. C. (1988). Effects of improving the reliability of the GPA on prediction generally and on comparative predictions for gender and race particularly.

arsides, T., \& Woodfield, R. (2003). Individual differences and undergraduate academic uccess: The roles of personality, intelligence, and application. Personality and

eving, A. M. (2007). Identifying the types of student and teacher behaviours associated
with teacher stress. Teaching and Teacher Education, 23, 624-640. ustafsson, J. -E., \& Balke, G. (1993). General and specific abilities as predictors of school rch, 28, 407-434.

Häusler, J., \& Sommer, M. (2006). Neuronale Netze: Nichtlineare Methoden der statistischen Urteilsbildung in der psychologischen Eignungsdiagnostik [Neural netZeitschift für Personalpsychologie 5,4-15.

Hell, B . Trapmann, S, \& Schuler, H. (2007). Eine Metaanalyse der Validität von fachspezifischen Studierfähigkeitstests im deutschsprachigen Raum [A meta-analytic investigation of subject-specific admission tests in German-speaking countries]. Empirische Pädagogik, 21, 251-270.

Hembree, R. (1988). Correlates, causes, effects and treatment of test anxiety. Review of Educational Research, 58, 47-77.

Horn, J. L., \& Noll, J. (1997). Human cognitive capabilities: Gf-Gc theory. In D. P. Flanagan, L. Genshaft, \& P. L. Harrison (Eds.), Contemporary intellectual assessment: Theory, 29-91). New York: The Guilford Press.

. Irvine, \& P. C. Kyllonen (Eds.), Item generation for test development (pp. 159-178). New Jersey: Lawrence Erlbaum Associates.

Hornke, L. F., Etzel, S., \& Küppers, A. (2000). Konstruktion und Evaluation eines adaptiven Matrizentests [Design and evaluation of an adaptive matrices test]. Diagnostica, 46, $182-188$

r. P. M. (1999). Cutoff criteria for fit indexes in covariance structure analysis:

Krammer, G., \& Pflanzl, B. (2015, Septembera). Studienerfolg in Lehramtsstudien: Eine 3jährige Längsschnittstudie zu Intelligenz, Gesundheits- und Erholungsverhalten und realistischen Berufserwartung |Academic achievement in teacher education: A 3-year longitudinal study on intelligence, recreational behaviour, and realistic job expectations]. (Paper presented at the 80. Annual Conference of the Arbeitsgruppe für Empirische Pädagogische Forschung (AEPF), Göttingen, DE).

Krammer, G., \& Pflanzl, B. (2015b). Faking von Persönlichkeitseigenschaften bei Zulassungsverfahren für Lehramtsstudien [Faking of personality measures for 29, 205-214.

Krapp, A. (1992). Konzepte und Forschungsansä̈ze zur Analyse des Zusammenhangs von Interesse, Lernen und Leistung [Concepts and approaches on the analysis of the relations between interests, learning, and performance]. In A. Krapp, \& M. Prenzel (Eds.), Interesse, Lernen, Leistung (pp. 9-52). Aschendorff: Múnster app, A., \& Prenzel, M. (2011). Research on interest in science: Theories, methods and
findings. International Journal of Science Education, 33, 27-50. 
Full Texts \& Dissemination

Kuncel, N. R., \& Hezlett, S. A. (2007). Standardized tests predict graduate student's success. Science, 315, 1080-1081.

Kuncel, N. R., Hezlett, S. A., \& Ones, D. S. (2001). A comprehensive meta-analysis of the predictive validity of the graduate record examinations: Implications for graduate student selection and pefformance. Pychological Bulletin, 127, 162-18

Kuncel, N. R. Hezlett S. A \& Ones, D. S. (2004). Academic performance, career potential creativity, and job performance: Can one construct predict them all? Journal of .

Kuncel, N. R., Wee, S., Serafin, L., \& Hezlett, S. A. (2010). The validity of the graduate record examination for master's and doctoral programs: A Educational and Psychological Measurement, 70, 340-352.

Lei, P. W. (2009). Evaluating estimation methods for ordinal data in structural equation

Marsh, H. W., Hau, K. T., \& Wen, Z. (2004). In search of golden rules: Comment on hypothesis testing approaches to setting cut off values for fit indexes and dangers in

Mayr J. (2012). Ein Lehramtsstudium beginnen? Ein Lehramtsstudium beginnen lassen? [Entering teacher education? Being admitted to teacher education?]. In B. Weyand, (pp. 38-57). Essen: Stifterverban

Mayr, J., \& Brandstätter, H. (1998). Lehrer/in werden? [Becoming a teacher?]. Wien Bundesministerium für Unterricht und kulturelle Angelegenheite

W. O. O., \& Hancock, G. R. (2008). Best practices in structural equation modeling. In.

O'Connor, M. C., \& Paunonen, S. V. (2007). Big five personality predictors of post-secondary academic performance. Personality and Individual Differences, 43, 971-990.

Paunonen, S. V.., \& Ashton, M. C. (2001). Big five factors and facets and the prediction of behavior. Journal of Personality and Social Psychology, 81, 524-53.9.

hillips, J. M. (1998). Effects of realistic job previews on multiple organizational outcomes: A meta-analysis. The Acodemy of Management Journal, 41, 673-690.

(2009). A meta-analysis of the five-factor model of personality and academic performance. Psychological Bulletin, 135, 322-338.

Premack. S. L. \& Wanous. J. P. (1985). A meta-anlysis of realistic job preview experiments. Journal of Applied Psychology, 70, 706-719.

Reeve, C. L. (2004). Differential ability antecedents of general and specific dimensions of declarative knowledge: More than g. Intelligence, 32, 621-652.

eve, C. L., \& Bonaccio, S. (2011). On the myth and the reality of the temporal validity degradation of general mental ability test scores. Intelligence, 39, 255-272.

Richardson, M.., Abraham, C.. \& Bond, R. (2012). Psychological correlates of university students' academic perforn
Rieder, E. M. (2011). Studierendenauswahl an der Pädagogischen Hochschule Steiermark IStudent selection at the University College of Teacher Education Styrial. (Dissertation). Verfügbar unter http://ub.uni-graz.at

Rosseel, Y. (2012). lavaan: An R package for structural equation modeling. Journal of Statistical Software, 48, 1-36.

oth, P. L., \& Clarke, R. L. (1998). Meta-analyzing the relation between grades and salary.

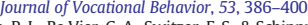

tionship between grades and job performance. Journal of Applied Psychology, $548-556$

Rynes, S. L. (1991). Recruitment, job choice, and posthire consequences. In M. D. Dunnette, \& L. M. Hough (Eds.), Handbook of industrial and organizational psychology

Savalei, V., \& Rhemtulla, M. (2013). The performance of robust test statistics with categorical data. British Jour

6hrerberuf. Analyse eines veränderungsbedürftigen Zustandes, 2. Auflage [Working only half of the day? Psy-
chological well-being in the teacher profession. An analyses of a state needing change]. Weinheim/Basel: Beltz

Schaarschmidt, U., \& Fischer, A. (2013). Manual inventory for personality assessment in sit-

uations (version 21 - revision 2). Mödling: Schuhfried.
Schaarschmidt, U., \& Kieschke, U. (2007). Gerüstet für den Schulalltag. Psychologische Unterstützungsangebote für Lehrerinnen und Lehrer [Being prepared for everyday school

Schmidt-Atzert, L., Deter, B., \& Jaeckel, S. (2004). Prädiktoren von Ausbildungserfolg: Allgemeine Intelligenz $(\mathrm{g})$ oder spezifische kognitive Fähigkeiten? [The prediction Zeitschrift für Personalpsychologie, 3, 147-158.

Strenze, T. (2007). Intelligence and socioeconomic success: A meta-analytic review of lon-

gitudinal research. Intelligence, 35, 401-426.
Trapmann, S., Hell, B., Weigand, S., \& Schuler, H. (2007). Die Validität von Schulnoten zur Vorhersage des Studienerfolgs - eine Metaanalyse [The validity of school grades for tice. Norwell MA., \& Glas, C. A. Wanous, J.P., Poland, T.D. Premack, S. L., \& Davis, S. (1992). The effects of met expectations on newcomer attitudes and behaviors: A review and meta-analysis. Journal of
Applied Psychology, 77, 288-297. 
6.2 Study 2: Faking of Personality Measures for College Admission in Teacher Education 


\title{
Faking von Persönlichkeitseigen- schaften bei Zulassungsverfahren für Lehramtsstudien
}

\author{
Georg Krammer und Barbara Pflanzl \\ Pädagogische Hochschule Steiermark, Graz
}

\begin{abstract}
Zusammenfassung: Werden selbstbeschreibende Verfahren in Zulassungsverfahren verwendet, stellt sich die Frage, wie sehr Faking diese verzerrt, d.h., wie sehr Personen sich vorteilhafter darstellen, als sie es tatsächlich sind. Bis dato wurde dies für angehende Lehrkräfte nicht untersucht. In der vorliegenden Studie wurde Faking in einem Zulassungsverfahren an einem Persönlichkeitsinventar zum Verhalten und Erleben in sozial-kommunikativen Situationen, Leistungs- und Erholungssituationen untersucht. 116 Lehramtsstudierende bearbeiteten ein halbes Jahr nach ihrer Zulassung dasselbe Persönlichkeitsinventar nochmals. Bei dieser Nacherhebung gab es eine experimentelle Manipulation mit drei Bedingungen, um 1) das tatsächliche Ausmaß an Faking, 2) die Reproduzierbarkeit von Faking und 3) das mögliche Manipu maß nur in 11 der 18 Subskalen reproduziert werden konnte. Bei Aufforderung zu faken, zeigte sich in 17 der 18 Subskalen Faking. Die Rangreihen waren umso verzerrter, je stärker Faking auftrat.
\end{abstract}

Schlüsselwörter: Faking, Persönlichkeit, Selektion, Zulassungsverfahren, IPS

Faking of Personality Measures for College Admission in Teacher Education

Abstract: Personality measures in student admission entail the question if applicants fake, i.e. distort their responses to be viewed more favorably than they actually are. This has not yet been investigated for student admission in teacher education. 116 students were given a personality inventory assessing social-communicative, achievement, and health-recreational behavior twice: once within student admission and half a year later. At this second measurement point, faking was manipulated experimentally to determine 1) the degree of faking within student admission, 2) if the faking of the student admission could be reproduced, and 3) how much faking was possible (= fakinggood). Results indicated that students faked within the student admission in 16 out of 18 subscales but could reproduce their degree of faking in only 11 out of 18 subscales. When instructed to fake, faking occurred in 17 out of 18 subscales. The students rank order was negatively affected by the degree of faking.

Keywords: faking, personality, selection, student admission, IPS

\section{Theoretischer Hintergrund}

Faking ist das Phänomen eigene Ergebnisse in selbstbeschreibenden Verfahren ins Vorteilhafte zu verzerren (McFarland \& Ryan, 2000) und stellt damit die Fairness von selbstbeschreibenden Verfahren bei Zulassungsverfahren in mehrerlei Hinsicht in Frage. Durch Faking werden nicht mehr die für die Zulassung intendierten Merkmale gemessen (Ziegler \& Bühner, 2009) und nicht alle der sich Bewerbenden verzerren ihre Selbstbeschreibungen in gleichem Maße (Birkeland, Manson, Kisamore, Brannick \& Smith, 2006). Dies kann dazu führen, dass nicht jene ausgewählt werden, welche am geeignetsten sind, sondern jene, welche sich am geeignetsten darstellen können. Deshalb darf Faking für die Vergabe von Lehramtsstudienplätzen nicht außer Acht gelassen werden, wenn selbstbeschreibende Verfahren zum Einsatz kommen.

Faking ist ein komplexer Prozess, der von vielen Faktoren beeinflusst wird (McFarland \& Ryan, 2000; Komar, Brown, Komar \& Robie, 2008; Tett, Freund, Christiansen, Fox \& Coaster, 2012). Diese Faktoren umfassen die eigenen Werte und Moralvorstellungen. Faking setzt Intelligenz und die Fähigkeit zu faken voraus. Darüber hinaus wird Faking von dem wahren Wert auf dem betroffenen 
Full Texts \& Dissemination

206 G. Krammer \& B. Pflanzl: Faking für Lehramtsstudienplätze

Merkmal beeinflusst, da niedrige Werte weiter nach oben hin verzerrt werden können als bereits hohe. Wie sehr Faking auftritt, hängt auch von der Attraktivität der angestrebten Stelle und der Höhe der Selektionsquote ab. Es ist auch entscheidend, welche Merkmale erhoben werden und für welche Art von Stelle diese erhoben werden. Neben diesen genannten Faktoren gibt es noch weitere, aber zusammengefasst können die Faktoren, die Faking beeinflussen, persönlich oder situativ sein (Ellingson \& McFarland, 2011).

Qualitative Studien zu Faking belegen, dass Personen in denselben Situationen unterschiedlich faken (Ziegler, 2011; König, Merz \& Trauffer, 2012). König et al. (2012) untersuchten die Kognitionen, die Faking zugrunde liegen, und stellten fest, dass Personen falsche Vorstellungen von selbstbeschreibenden Verfahren haben. U.a. gingen Personen davon aus, dass sich Inkonsistenzen in ihren Einzelantworten negativ auf ihr Gesamtergebnis auswirken würden. Diese Kognitionen legen nahe, dass Personen nicht wissen, dass die Items einer Skala in der Regel einfach summiert werden und, aufgrund mangelnder Reliabilität, keine einzelnen Items miteinander verglichen werden.

Studien belegen, dass es sich bei Faking um ein tatsächliches Problem handelt. Die Meta-Analyse zu Faking von Viswesvaran und Ones (1999) bestätigt, dass Personen in der Lage sind zu faken (abhängig vom Design $0.48 \leq$ $d \leq 0.65$ bzw. $0.47 \leq d \leq 0.93$ ). Birkeland et al. (2006) zeigten, dass in Bewerbungssituationen Faking ebenfalls auftritt, jedoch in verringertem Ausmaß $(0.11 \leq d \leq 0.45)$. Faking tritt auch über verschiedene Erhebungsmethoden hinweg auf. Selbst z.B. Auswahlgespräche - welche zur Studienplatzvergabe akzeptiert (Hell \& Schuler, 2005) und aussagekräftig (Hell, Trapmann, Weigand \& Schuler, 2007) sind - sind für Faking anfällig (Levashina \& Campion, 2006; Levashina \& Campion, 2007). Sogar bei Integritätstests und Tests mit biographischen Inhalten konnte Faking nachgewiesen werden (McFarland \& Ryan, 2000). Auch wenn Persönlichkeitsmerkmale projektiv oder semiprojektiv statt selbstbeschreibend erhoben werden, tritt Faking auf (Ziegler, Schmidt-Atzert, Bühner \& Krumm, 2007).

Der ausschließliche Einsatz von kognitiven Leistungstests könnte Faking in Zulassungsverfahren vermeiden. Allgemeine Intelligenz ist einer der besten Prädiktoren für späteren Berufserfolg (Schmidt \& Hunter, 2004). Deshalb werden Intelligenztests und/oder fachspezifische Wissenstests in Zulassungsverfahren eingesetzt und haben sich dafür bewährt (Kuncel, Hezlett \& Ones, 2001; Kuncel et al., 2005; Kuncel \& Hezlett, 2007). Auch fachspezifische Leistungstests wie Studierfähigkeitstests sind für Zulassungsverfahren anerkannt (Kuncel et al., 2001; Hell, Trapmann \& Schuler, 2007).

Diese Praxis der Verwendung von kognitiven Leistungstests in Zulassungsverfahren könnte auf die Vergabe von Lehramtsstudienplätzen übertragen werden. Päßler, Hell und Schuler (2011) weisen darauf hin, dass kognitive Merkmale für die Studien- und Berufsbewährung bei
Lehrkräften relevant sind. Die Bedeutsamkeit von kognitiven Merkmalen für Lehrkräfte zeigt sich bereits beim Studienerfolg (Kaub et al., 2012; Krammer, 2014). Zusätzlich ist das Fachwissen der Lehrkräfte eine notwendige, wenn auch nicht hinreichende, Voraussetzung für eine hohe Qualität des Unterrichts (Baumert \& Kunter, 2011).

Persönlichkeitsmerkmale sollten jedoch bei Zulassungsverfahren nicht außer Acht gelassen werden, da sie im Allgemeinen als Prädiktoren für Erfolg im Studium und im Beruf gelten. Einzelne Facetten der Big Five beeinflussen den Studienerfolg (Trapmann, Hell, Hirn \& Schuler, 2007; Poropat, 2009) und Berufserfolg (Judge, Higgins, Thoresen \& Barrick, 1999). Sie liefern dazu in beiden Fällen einen inkrementellen Beitrag über die Intelligenz hinaus (Judge et al., 1999; Farsides \& Woodfield, 2003; Ziegler, Danay, Schölmerich \& Bühner, 2010). Persönlichkeitsmerkmale können damit ein Mehrgewinn für Zulassungsverfahren sein, auch wenn durch deren Verwendung Faking eine zu adressierende Problematik wird (Kuncel \& Hezlett, 2010).

Während es für den Lehrerberuf fraglich ist, ob es «die Lehrerpersönlichkeit» wirklich gibt (Bromme \& Haag, 2004), gelten Persönlichkeitsmerkmale und berufliches Interesse als Prädiktoren für die Studien- und Berufsbewährung bei Lehrkräften (Hanfstingl \& Mayr, 2007). Emotionale Stabilität, Extraversion und Gewissenhaftigkeit haben sich als relevant für die Bewährung im Lehramtsstudium und Lehrerberuf gezeigt (Mayr, 2011). Auch berufliches Interesse steht in Zusammenhang mit dem Studienerfolg (Hanfstingl \& Mayr, 2007; Kaub et al., 2012). Demnach ist es nicht zielführend, künftige Lehrkräfte nur aufgrund von kognitiven Leistungstests auszuwählen. Bis dato gibt es jedoch noch keine Studien, die Faking bei Zulassungsverfahren zur Vergabe von Lehramtsstudienplätzen thematisieren.

\section{Die vorliegende Studie}

Klassische Fakingstudien beruhen zumeist auf einer experimentellen Manipulation: Personen werden aufgefordert, sich so ideal wie möglich darzustellen (= Faking-good); dies wird im Anschluss mit einer Kontrollbedingung verglichen. Griffith, Chmielowski und Yoshita (2007) untersuchten Faking in der Personalauswahl, indem dieses Untersuchungsdesign einer tatsächlichen Bewerbungssituation folgte. Für ein ähnliches Design wie Griffith et al. plädierten Komar et al. (2008), um Faking in Bewerbungssituationen zu untersuchen. Griffin und Wilson (2012) verwendeten dieses Design ohne experimentelle Manipulation, um Faking in einem Inventar zu den Big Five bei der Selektion von Medizinstudierenden zu untersuchen. In der vorliegenden Studie wurden diese Ideen aufgegriffen: Es werden hierbei Daten aus einem Zulassungsverfahren zur Studienplatzvergabe für Lehramtsstudien mit Daten einer anschließenden experimentellen Manipulation verglichen. 
Full Texts \& Dissemination

G. Krammer \& B. Pflanzl: Faking für Lehramtsstudienplätze

Als Erstes war es von Interesse herauszufinden, inwiefern Bewerbende für Lehramtsstudien Persönlichkeitsmerkmale faken. Sie sollten sich in den für den Lehrerberuf relevant wahrgenommenen Persönlichkeitsmerkmalen besser darstellen, als sie es sind (Birkeland et al., 2006; Ziegler, 2011). In einer Faking-good-Bedingung sollten sie sich besser darstellen als beim Zulassungsverfahren, da in tatsächlichen Bewerbungssituationen weniger Faking auftritt, als möglich wäre (Birkeland et al., 2006). In der vorliegenden Studie war ein Persönlichkeitsinventar zum Verhalten und Erleben in sozial-kommunikativen Situationen, Leistungssituationen und Gesundheits- und Erholungssituationen Teil eines Zulassungsverfahrens für Lehramtsstudien: das Verhalten und Erleben in 1) sozialkommunikativen Situationen, da sich Extraversion als relevantes Kriterium für die Bewährung im Lehramtsstudium und Lehrerberuf herausgestellt hat (Mayr, 2011); 2) Leistungssituationen, da es im Berufsalltag häufig zu Situationen mit besonderen Leistungs- und Handlungserfordernissen kommt, wie z.B. der Umgang mit schwierigen Schülerinnen und Schülern (Schaarschmidt, 2004); 3) Gesundheits- und Erholungssituationen, da Lehrkräfte stark Burn-out gefährdet sind (Unterbrink et al., 2007). Schaarschmidt und Fischer (2013) zeigten die Wichtigkeit dieser Merkmale für den Lehrerberuf durch einen Vergleich mit für den Lehrerberuf relevanten Persönlichkeitsvoraussetzungen (Mayr \& Brandstätter, 1998). Sie zeigten auch, dass Lehrkräfte und Lehramtsstudierende vergleichbare Ausprägungen erzielen, diese sich jedoch von z.B. Psychologiestudierenden unterscheiden. Zudem besteht ein Zusammenhang dieser Merkmale mit der Studienleistung von Lehramtsstudierenden (Krammer, 2014).

Neben den praktischen Implikationen von Faking bedarf es aus wissenschaftlicher Sicht noch eines Erkenntnisgewinns zum Faking-Prozess (Burns \& Christiansen, 2011; Griffith \& Peterson, 2011). Ist Faking ein Prozess, der reproduziert werden kann, oder ist dieser bei Zulassungsverfahren derart komplex, dass er es nicht werden kann? Als Zweites interessierte daher, inwieweit das Faking eines Zulassungsverfahrens reproduziert werden kann. Aufgrund der Komplexität dieses Prozesses wird angenommen, dass die Bewerbenden dies nicht können.

Die Verzerrung der resultierenden Rangreihen durch Faking ist für die Praxis von Zulassungsverfahren das größte Problem. Diese Verzerrung beeinflusst unweigerlich auch die Selektion (Rosse, Stecher, Miller \& Levin, 1998). Als Drittes interessierte daher, inwiefern das Ausmaß an Faking die Rangreihen der Bewerbenden verändert. Da Untersuchungen zufolge Faking die Rangreihen in Selektionsprozessen beeinflusst (Rosse et al., 1998; Winkelspecht, Lewis \& Thomas, 2006), sollten diese Rangreihen umso mehr verändert werden, je mehr Faking auftritt.

Zusammengefasst wurde in der vorliegenden Studie das Faking in einem Persönlichkeitsinventar bei einer Studienplatzvergabe von Lehramtsstudien untersucht. Es wurden Daten eines Zulassungsverfahrens mit einer anschließen- den experimentellen Manipulation verglichen. Dafür wurden folgende Hypothesen aufgestellt: 1) Die sich für Lehramtsstudien Bewerbenden stellen sich besser dar, als sie tatsächlich sind und können sich noch besser darstellen. 2) Sie können ihr Ausmaß an Faking nicht reproduzieren und 3) deren Rangreihen verändern sich umso mehr, je mehr Faking auftritt.

\section{Methode}

An der Pädagogischen Hochschule Steiermark (PHSt) sind die Studienplätze beschränkt. In der Regel werden pro Studienjahr ca. $60 \%$ der Personen, die sich auf einen Studienplatz bewerben, zugelassen. Einerseits beinhaltet das Zulassungsverfahren Leistungsmerkmale: die Stimm- und Sprechleistung, die Kenntnisse der deutschen Sprache, die allgemeine Intelligenz und ggf. studiengangsspezifische Fachtests. Andererseits beinhaltet es Persönlichkeitsmerkmale: das Verhalten und Erleben in sozial-kommunikativen Situationen, Leistungssituationen und Gesundheits- und Erholungssituationen (Schulz-Kolland, Krammer, Rottensteiner \& Weitlaner, 2014). Die Persönlichkeitsmerkmale werden durch das Inventar zur Persönlichkeitsdiagnostik in Situationen (IPS: Schaarschmidt \& Fischer, 2013) erhoben.

\subsection{IPS}

Schaarschmidt und Fischer (2013, S.5) empfehlen das IPS für «Berufsgruppen, die sich durch ein höheres Maß psychosozialer Beanspruchung und durch akzentuierte Anforderungen an das sozial-kommunikative Verhalten auszeichnen», und nennen hierfür explizit Lehrkräfte. In diesem Sinne führen sie auch Reliabilitäten und Validitäten u.a. für Lehrkräfte und Lehramtsstudierende an. Das IPS erhebt drei Faktoren höherer Ordnung: das Verhalten und Erleben in 1) sozial-kommunikativen Situationen (6 Subskalen), 2) Leistungssituationen (6 Subskalen) und 3) Gesundheits- und Erholungssituationen (3 Subskalen). Für jede Subskala wird eine prototypische Situation vorgegeben und es gilt, das eigene Verhalten und Erleben darin einzuschätzen. Eine Subskala des sozial-kommunikativen Verhaltens und Erlebens ist z.B. die Rücksichtnahme bei sozialer Verantwortung, wofür eine Situation mit sozialer Verantwortung vorgegeben wird («Mir werden Aufgaben übertragen, bei denen ich ständig mit anderen Menschen zu tun habe und Verantwortung für diese Menschen wahrnehmen muss.») und es gilt, Fragen bzgl. der eigenen Rücksichtnahme zu beantworten (z. B.: «Wahrscheinlich werde ich in solch einer Situation viel Verständnis zeigen.»). Eine weitere Subskala ist z.B. die Aktivität in vertrauter kommunikativer Situation: Hier wird eine vertraute und kommunikative Situation vorgegeben und es gilt, Fragen bzgl. der eigenen Aktivität zu beantworten. Eine Subskala für das Verhalten und Erleben in Leistungssituationen ist 
Full Texts \& Dissemination

208 G. Krammer \& B. Pflanzl: Faking für Lehramtsstudienplätze

z. B. die Beharrungstendenz bei Umstellungserfordernis, wofür eine Situation mit Umstellungserfordernis vorgegeben wird und Fragen bzgl. der eigenen Beharrungstendenz zu beantworten sind. Die Items sind auf einer 4-stufigen Skala zu beantworten (stimmt gar nicht - stimmt genau). Zusätzlich zu diesen 15 Subskalen wird die Zufriedenheit mit dem eigenen Verhalten und Erleben in jeder dieser Situationen 5-stufig (sehr unzufrieden - sehr zufrieden) erfragt. Daraus ergeben sich drei Zufriedenheitsskalen, eine pro Faktor höherer Ordnung. Damit besteht das IPS aus 18 Subskalen (s. Tabelle 1 für eine Auflistung aller Subskalen).

Schaarschmidt und Fischer (2013) berichten von der zusammengesetzten Eichstichprobe für ihre 18 Skalen interne Konsistenzen mit Cronbachs $\alpha$ von .71 bis .91 und für Lehrkräfte und Lehramtsstudierende von .69 bis .91 . In Tabelle 1 sind für alle Skalen die internen Konsistenzen der vorliegenden Stichprobe beim Zulassungsverfahren angeführt. ${ }^{1}$ Sie fielen geringer als die berichteten aus. Teilweise fielen sie für Persönlichkeitsmerkmale, die mit geringen Itemzahlen erhoben werden, nicht sehr zufriedenstellend aus.

\subsection{Design und experimentelle Manipulation}

Das IPS wurde ein halbes Jahr nach dem Zulassungsverfahren (t1) nochmals bearbeitet (t2). Zu t2 wurde Faking («Faking-good»), Faking wie beim Zulassungsverfahren («wie-damals») und, als Baseline, kein Faking («honest») experimentell mittels Instruktionen induziert. Bei Fakinggood wurde instruiert, nicht wahrheitsgetreu zu antworten, sondern sich so geeignet wie möglich darzustellen, um ein Lehramtsstudium an der PHSt beginnen zu dürfen. Bei wie-damals wurde instruiert, das IPS wie zu t1 zu bearbeiten. Es wurde dazu aufgefordert, sich in die Situation des damaligen Zulassungsverfahrens hineinzuversetzen und die Items so zu beantworten, als würde jetzt am $\mathrm{Zu}$ lassungsverfahren teilgenommen werden. Bei der honestBedingung erfolgte nur eine Instruktion für das IPS und es wurden keinerlei Hinweise auf das Zulassungsverfahren gegeben.

\subsection{Stichprobe}

Für die Nacherhebung wurde unter jenen Lehramtsstudierenden rekrutiert, die für das betreffende Studienjahr das Zulassungsverfahren durchlaufen hatten. 563 Bewerbende hatten das IPS vollständig bearbeitet, davon wurden 286 als ordentliche Studierende des Primar- oder SekundarStudiengangs aufgenommen. 121 Studierende nahmen an der vorliegenden Studie teil. Die Analysen beziehen sich auf die 116 Personen, deren Daten mit t1 verknüpft werden konnten. ${ }^{2}$ Dies entspricht einer Rücklaufquote von $40.6 \%$, bezogen auf das betreffende Studienjahr. Zu t2 wurde, gleich wie zu t1, in Kleingruppen (8-12) getestet. Diese Kleingruppen wurden zufällig den drei Bedingungen zugeteilt. Die Stichprobengrößen der honest-, wiedamals- bzw. Faking-good-Bedingung betrugen 36, 39 bzw. 41 und waren damit vergleichbar groß $\left(\chi^{2}[2]=0.33\right.$, $p=.85)$. Da die Kleingruppen den Bedingungen zufällig zugeteilt wurden, wurde geprüft, ob die Kleingruppen in sich überzufällig homogen waren: ICC (one-way, single) wurden für jede IPS-Skala berechnet. 7 dieser ICC fielen über 0 aus, der höchste war .09 in der IPS-Skala Rücksicht nahme bei sozialer Verantwortung. Univariate Varianzanalysen mit den Kleingruppen als zufällige Effekte zeigte, dass alle ICC nur zufällig von 0 verschieden waren (alle $p>.07)$.

Die Lehramtsstudierenden (davon $72.4 \%$ Studentinnen) waren zu t2 zwischen 18 und 43 Jahre alt $(M=23.9, S D=$ 4.9). Die Geschlechterverteilung war vergleichbar mit der Verteilung innerhalb der neu zugelassenen Studierenden des betreffenden Studienjahrs (Studentinnen/Studenten $\left.70.8 \% / 29.2 \%, \chi^{2}[1]=1.4, p=.71\right)$. Das Geschlecht war nicht mit den Bedingungen $\left(\chi^{2}[2]=1.18, p=.56, V=.1\right)$ und nicht mit dem Alter $\left(F[2,113]=0.44, p=.65, \eta^{2}<\right.$ $.01)$ konfundiert. Alle Studierenden waren im 2. Semester ihres jeweiligen Studiums. Der Studienfortschritt war damit konstant gehalten. Schließlich zeigte sich auch für den Studiengang keine Konfundierung mit den Bedingungen $\left(\chi^{2}[2]=0.39, p=.82, V=.06\right): 55$ Lehramtsstudierende nahmen aus dem Sekundar-Studiengang $(=47.4 \%)$ und 61 aus dem Primar-Studiengang $(=52.6 \%)$ teil.

\subsection{Statistische Auswertung}

Das Ausmaß an Faking wurde über die Mittelwertunterschiede operationalisiert. Die Hypothesen dazu wurden varianzanalytisch geprüft. Hierfür war die Wechselwirkung zwischen Zeitpunkt und Bedingung von Interesse. Die Haupteffekte waren weder für die Hypothesen relevant, noch sollten sie bei einer hypothesenkonformen Wechselwirkung interpretiert werden, da diese hybrid wäre. Die Normalverteilung der Stichprobenmittelwerte wurde wegen der Stichprobengrößen angenommen, die Varianzhomogenität kann bei vergleichbarer GruppengröBe vernachlässigt werden und Sphärizität ist erst ab drei Messzeitpunkten relevant (Bortz \& Schuster, 2010).

Um eine $\alpha$-Fehler-Inflation zu vermeiden, wurde so global wie möglich und bei gegebenen Effekten immer ein Schritt lokaler getestet: zuerst eine MANOVA über alle

1 Die internen Konsistenzen jeder Bedingung zur Nacherhebung sind im Elektronischen Supplement Tabelle 2-4 angeführt.

2 Um Hinweise auf Selektionseffekte zu liefern, wurde verglichen, ob die vorliegenden 116 Personen sich von den 563 Bewerbenden in den IPS-Skalen unterschieden. Die mittlere Stärke des Effekts war $d=0.08$. Einzig der höchste Effekt $(d=0.2)$ war signifikant $(t[115]=2.33, p=$ $.02)$

Z. Pädagog. Psychol. 29 (3-4) @ 2015 Verlag Hans Huber, Hogrefe AG, Bern 
Full Texts \& Dissemination

G. Krammer \& B. Pflanzl: Faking für Lehramtsstudienplätze

IPS-Skalen, bei signifikanter Wechselwirkung anschlieBend ANOVAs für die einzelnen IPS-Skalen. Für Skalen mit signifikanter Wechselwirkung wurde der Tukey HSD Posttest, ein konservativer Posttest (Field, Miles \& Field, 2012), durchgeführt. Im Posttest wurden zuerst die Bedingungen zu t1 verglichen, anschließend die Bedingungen zwischen t1 und t2. Nach diesem Vergleich innerhalb der Bedingungen wurde noch ein Vergleich zwischen den Bedingungen zu $\mathrm{t} 2$ vorgenommen. Es wurde anhand der honest-Bedingung zu $\mathrm{t} 2$ errechnet, wo der Cut-off in den jeweiligen IPS-Skalen für zwei Selektionsquoten $(50 \%$ und $70 \%$ ) sein müsste. Für diese Cut-offs wurde dann geprüft, wie viel Prozent der Lehramtsstudierenden in den drei Bedingungen aufgenommen worden wären.

Für die Hypothese bezüglich des Einflusses von Faking auf die Rangreihen wurde das Ausmaß an Faking mit den Rangreihenkorrelationen in Beziehung gesetzt. Von Interesse war, wie sehr eine Veränderung im Ausmaß an Faking mit einer Veränderung in der Höhe der Rangkorrelationen einherging. Das Ausmaß an Faking wurde über die Effektstärken der Mittelwertunterschiede zwischen $\mathrm{t} 1$ und $\mathrm{t} 2$ in den Bedingungen operationalisiert. Diese Effektstärken wurden mittels Korrelation in Beziehung zu den Rangreihenkorrelationen gesetzt.

Das übliche Signifikanzniveau von 5\% wurde verwendet, da es an Studien mangelt, aus welchen zu erwartende Effektstärken von Faking für Lehramtsstudienplätze im IPS ableitbar wären. Das Signifikanzniveau wurde angepasst, um ggf. einem $\alpha$-Fehler bzw. $\beta$-Fehler entgegenzuwirken. Dies betraf den Posttestvergleich zwischen den Bedingungen zu t1 als auch die Rangreihenkorrelationen. Jener Posttestvergleich prüfte, ob keine Unterschiede bestanden. Daher sollte das Signifikanzniveau erhöht werden, um damit den $\beta$-Fehler zu verringern. Es wurde auf $10 \%$ gesetzt (Bortz und Schuster [2010] schlagen 25\% vor, jedoch für einen einzigen Vergleich, was bei vorliegender Anzahl an Einzelvergleichen zu liberal wäre). Für die Rangreihenkorrelationen wurde das Signifikanzniveau auf $1 \%$ gesenkt, aufgrund deren Anzahl und dem damit einhergehenden $\alpha$-Fehler

\section{Ergebnisse}

Die Mittelwerte und Standardabweichungen aller IPSSkalen in den drei Bedingungen zu t1 und zu t2 sind in Tabelle 1 angeführt. Die Interkorrelationen zu t1 fielen zwischen -.49 und .63 aus. $^{3}$ Die Höhe der Korrelationen wurde nach Bortz und Döring (2006) klassifiziert: 4 Korrelationen waren vernachlässigbar $(r<.1), 67$ klein $(.1 \leq$ $r<.3), 73$ mittel $(.3 \leq r<.5)$ und 9 groß $(r \geq .5)$. Die Interkorrelationen bestätigten, dass die 18 IPS-Skalen nicht 18 getrennte Konstrukte waren. Es war daher zulässig, über die IPS-Skalen hinweg multivariat zu testen (Backhaus, Erichson, Plinke \& Weiber, 2006). Hierfür zeigte sich eine signifikante Wechselwirkung $(F[36,194]=3.87, p<.01$, $\lambda=.26, \eta^{2}=.42$ ) mit signifikanten Haupteffekten (Bedingung: $F[36,194]=1.9, p<.01, \lambda=.53, \eta^{2}=.26$; $\mathrm{t} 1-\mathrm{t} 2$ : $\left.F[18,96]=3.81, p<.01, \lambda=.58, \eta^{2}=.42\right)$. Anschließend wurden die einzelnen IPS-Skalen analysiert. Alle Wechselwirkungen waren signifikant mit dem geringsten Effekt bei Rücksichtnahme bei sozialer Verantwortung $(F[2,113]$ $\left.=5.35, p<.01, \eta^{2}=.08\right)$ und dem größten bei Zufriedenheit mit dem Leistungsverhalten $(F[2,113]=59, p<.01$, $\eta^{2}=.51$ ). Bei diesen beiden Skalen war der Haupteffekt der Bedingung einmal nicht signifikant und einmal signifikant $\left(F[2,113]=1.48, p=.23, \eta^{2}=.03\right.$ bzw. $F[2,113]=$ $\left.8.28, p<.01, \eta^{2}=.13\right)$. Der zweite Haupteffekt war bei beiden Skalen nicht signifikant $(F[1,113]=1.75, p=.19$, $\eta^{2}=.02$ bzw. $\left.F[1,113]<0.01, p=.96, \eta^{2}<.01\right)$. Die Posttests fanden für 14 der 18 IPS-Skalen keine Unterschiede der Bedingungen zu t1 (alle $p>.19$, alle $d<0.28$ ). In den vier IPS-Skalen Entspannungsfähigkeit nach dem Arbeitstag, Stabilität bei stressvoller Anforderung, Zufriedenheit mit dem Gesundheits- und Erholungsverhalten bzw. Zufriedenheit mit dem Leistungsverhalten unterschieden sich die Bedingungen Faking-good und honest bereits zu t1 $(p=.07,=.02,=.08$ bzw. $=.09) ;$ Faking-good war stets niedriger als honest $(d=0.42,=0.60,=0.42 \mathrm{bzw} .=0.37)$. Zusätzlich zeigte sich bei Stabilität bei stressvoller Anforderung ein Unterschied zwischen der wie-damals- und der honest-Bedingung ( $p=.096, d=0.43$ ). Danach wurden $\mathrm{t} 1$ und $\mathrm{t} 2$ innerhalb der Bedingungen verglichen (s. Tabelle 1 für $p$-Werte und Effektstärken). Die Vorzeichen der Effektstärken fielen für alle IPS-Skalen hypothesenkonform aus: In der honest- und wie-damals-Bedingung war $\mathrm{t} 1$ vorteilhafter ausgeprägt als t 2 ; in der Faking-good-Bedingung war $\mathrm{t} 2$ vorteilhafter als $\mathrm{t} 1$. In der honest-Bedingung gab es Effekte zwischen $d=-0.27$ und $d=-1.87$, mit dem größten Effekt bei Stabilität bei stressvoller Anforderung. In der wie-damals-Bedingung zeigten sich Effekte zwischen $d=-0.09$ und $d=-0.91$, mit dem größten Effekt bei Zufriedenheit mit dem Leistungsverhalten. In der Fakinggood-Bedingung betrugen die Effekte zwischen $d=0.35$ und $d=1.73$, mit dem größten Effekt bei Selbstvertrauen bei Prüfungsanforderung. Diese Unterschiede waren in 16, 11 bzw. 17 IPS-Skalen der honest-, wie-damals- bzw. Faking-good-Bedingung signifikant.

Nach diesem Vergleich innerhalb der Bedingungen wurden die Bedingungen zu t2 verglichen. Es wurde für jede IPS-Skala anhand der honest-Bedingung ermittelt, wo Cut-offs zu setzen wären, um $50 \%$ bzw. $70 \%$ zu selektieren. Anschließend wurde für diese Cut-offs geprüft, wie viel Prozent der Studierenden in der wie-damals- und Faking-good-Bedingung aufgenommen worden wären (s. Tabelle 1). Z.B. in der IPS-Skala Aktivität in vertrauter

3 Alle Interkorrelationen sind im Elektronischen Supplement angeführt. Siehe Tabelle 1 für die Interkorrelationen zu t1 und Tabelle 2-4 für die Interkorrelationen innerhalb der Bedingungen zu t1 und $\mathrm{t} 2$. 
Full Texts \& Dissemination

$210 \quad$ G. Krammer \& B. Pflanzl: Faking für Lehramtsstudienplätze

Tabelle 1

Mittelwert (M) und Standardabweichung (SD) der IPS-Skalen (Itemanzahl und Cronbachs a zu t1 in Klammer nach der Skalenbezeichnung) zur Zulassung ( $\mathrm{t} 1$ ) und Nacherhebung ( $\mathrm{t} 2$ ) in den drei Bedingungen (honest, wie-damals und Faking-good). Für den t1-t2-Vergleich sind Cohens $d$ (d) und die Signifikanz nach Tukey HSD (p(T)) angegeben, für den t1-t2-Zusammenhang deren Rangkorrelation $(\mathrm{r})$ und Signifikanz $(\mathrm{p}(\mathrm{r}))$. Für zwei Selektionsquoten $(50 \%$ und $70 \%)$ ist angegeben, wie viel Prozent bei einem entsprechenden Cut-off der honest-Bedingung in den drei Bedingungen aufgenommen worden wären.

\begin{tabular}{|c|c|c|c|c|c|c|c|c|c|c|}
\hline & \multicolumn{2}{|c|}{$\mathrm{t} 1$} & \multicolumn{2}{|c|}{$\mathrm{t} 2$} & \multirow[b]{2}{*}{$d$} & \multirow[b]{2}{*}{$p(T)$} & \multirow[b]{2}{*}{$r$} & \multirow[b]{2}{*}{$p(r)$} & \multirow[b]{2}{*}{$50 \%$} & \multirow[b]{2}{*}{$70 \%$} \\
\hline & $M$ & $S D$ & $M$ & $S D$ & & & & & & \\
\hline \multicolumn{11}{|c|}{ Aktivität in vertrauter kommunikativer Situation $(5 ; \alpha=.67)$} \\
\hline honest & 3.49 & 0.31 & 3.31 & 0.52 & -0.58 & $<.01$ & .45 & $<.01$ & 44 & 19 \\
\hline wie-damals & 3.47 & 0.34 & 3.35 & 0.37 & -0.61 & $<.05$ & .69 & $<.01$ & 44 & 15 \\
\hline Faking-good & 3.49 & 0.34 & 3.66 & 0.30 & 0.57 & $<.01$ & .13 & .41 & 73 & 49 \\
\hline \multicolumn{11}{|c|}{ Beharrungstendenz bei Umstellungserfordernis $(5 ; \alpha=.58)$} \\
\hline honest & 1.88 & 0.36 & 2.23 & 0.45 & 1.01 & $<.01$ & .33 & .05 & 36 & 28 \\
\hline wie-damals & 1.91 & 0.42 & 2.13 & 0.50 & 0.56 & $<.01$ & .24 & .14 & 51 & 36 \\
\hline Faking-good & 1.92 & 0.48 & 1.43 & 0.49 & -1.36 & $<.01$ & .36 & .02 & 95 & 88 \\
\hline \multicolumn{11}{|c|}{ Durchsetzung in einer Führungsrolle $(5 ; \alpha=.71)$} \\
\hline honest & 3.41 & 0.36 & 3.29 & 0.43 & -0.47 & .11 & .70 & $<.01$ & 31 & 22 \\
\hline wie-damals & 3.40 & 0.43 & 3.29 & 0.46 & -0.37 & .10 & .59 & $<.01$ & 33 & 26 \\
\hline Faking-good & 3.39 & 0.44 & 3.66 & 0.41 & 0.81 & $<.01$ & .38 & .02 & 73 & 59 \\
\hline \multicolumn{11}{|c|}{ Empfindlichkeit bei sozialer Frustration $(6 ; \alpha=.60)$} \\
\hline honest & 1.96 & 0.33 & 2.32 & 0.50 & 1.13 & $<.01$ & .48 & $<.01$ & 42 & 25 \\
\hline wie-damals & 2.06 & 0.49 & 2.10 & 0.45 & 0.15 & .54 & .57 & $<.01$ & 69 & 59 \\
\hline Faking-good & 1.93 & 0.43 & 1.45 & 0.39 & -1.56 & $<.01$ & .39 & .01 & 98 & 98 \\
\hline \multicolumn{11}{|c|}{ Engagement bei hoher Leistungsanforderung $(5 ; \alpha=.79)$} \\
\hline honest & 3.09 & 0.50 & 2.79 & 0.65 & -0.72 & $<.01$ & .52 & $<.01$ & 47 & 28 \\
\hline wie-damals & 3.11 & 0.47 & 2.87 & 0.44 & -0.78 & .02 & .58 & $<.01$ & 49 & 15 \\
\hline Faking-good & 3.04 & 0.62 & 3.65 & 0.61 & 1.03 & $<.01$ & -.09 & .56 & 93 & 85 \\
\hline \multicolumn{11}{|c|}{ Entspannungsfähigkeit nach dem Arbeitstag $(5 ; \alpha=.64)$} \\
\hline honest & 3.52 & 0.36 & 3.27 & 0.49 & -0.77 & $<.01$ & .53 & $<.01$ & 36 & 17 \\
\hline wie-damals & 3.43 & 0.41 & 3.30 & 0.42 & -0.42 & .06 & .46 & $<.01$ & 38 & 10 \\
\hline Faking-good & 3.36 & 0.40 & 3.81 & 0.23 & 1.62 & $<.01$ & .31 & .05 & 88 & 71 \\
\hline \multicolumn{11}{|c|}{ Aktives Erholungsverhalten in der Freizeit $(5 ; \alpha=.56)$} \\
\hline honest & 3.65 & 0.24 & 3.57 & 0.43 & -0.27 & .21 & .35 & .04 & 47 & 25 \\
\hline wie-damals & 3.58 & 0.37 & 3.56 & 0.42 & -0.10 & .67 & .67 & $<.01$ & 49 & 28 \\
\hline Faking-good & 3.64 & 0.35 & 3.91 & 0.18 & 1.05 & $<.01$ & .25 & .12 & 88 & 73 \\
\hline \multicolumn{11}{|c|}{ Gesundheitsvorsorge bei Warnsignalen $(5 ; \alpha=.69)$} \\
\hline honest & 3.57 & 0.37 & 3.11 & 0.45 & -1.44 & $<.01$ & .17 & .32 & 42 & 19 \\
\hline wie-damals & 3.49 & 0.46 & 3.21 & 0.63 & -0.83 & $<.01$ & .61 & $<.01$ & 49 & 36 \\
\hline Faking-good & 3.53 & 0.38 & 3.82 & 0.30 & 1.11 & $<.01$ & .39 & .01 & 93 & 90 \\
\hline \multicolumn{11}{|c|}{ Karriere- und Risikobereitschaft bei beruflicher Herausforderung $(5 ; \alpha=.79)$} \\
\hline honest & 3.57 & 0.34 & 3.25 & 0.60 & -0.97 & $<.01$ & .60 & $<.01$ & 42 & 22 \\
\hline wie-damals & 3.48 & 0.42 & 3.25 & 0.47 & -0.63 & $<.01$ & .45 & $<.01$ & 36 & 18 \\
\hline Faking-good & 3.52 & 0.44 & 3.79 & 0.35 & 0.85 & $<.01$ & .51 & $<.01$ & 83 & 71 \\
\hline \multicolumn{11}{|c|}{ Konfrontationstendenz in sozialer Konfliktsituation $(9 ; \alpha=.66)$} \\
\hline honest & 1.53 & 0.29 & 1.90 & 0.51 & 1.33 & $<.01$ & .67 & $<.01$ & 47 & 25 \\
\hline wie-damals & 1.50 & 0.34 & 1.67 & 0.35 & 0.78 & $<.01$ & .64 & $<.01$ & 69 & 33 \\
\hline Faking-good & 1.51 & 0.32 & 1.21 & 0.35 & -0.98 & $<.01$ & .45 & $<.01$ & 98 & 93 \\
\hline
\end{tabular}

Z. Pädagog. Psychol. 29 (3-4) @ 2015 Verlag Hans Huber, Hogrefe AG, Bern 
Full Texts \& Dissemination

G. Krammer \& B. Pflanzl: Faking für Lehramtsstudienplätze

Tabelle 1

Mittelwert (M) und Standardabweichung (SD) der IPS-Skalen (Itemanzahl und Cronbachs $\alpha$ zu tl in Klammer nach der Skalenbezeichnung) zur Zulassung (t1) und Nacherhebung ( $\mathrm{t} 2$ ) in den drei Bedingungen (honest, wie-damals und Faking-good). Für den t1-t2-Vergleich sind Cohens $d$ (d) und die Signifikanz nach Tukey HSD $(\mathrm{p}(\mathrm{T})$ ) angegeben, für den t1-t2-Zusammenhang deren Rangkorrelation (r) und Signifikanz (p(r)). Für zwei Selektionsquoten $(50 \%$ und $70 \%)$ ist angegeben, wie viel Prozent bei einem entsprechenden Cut-off der honest-Bedingung in den drei Bedingungen aufgenommen worden wären. (Fortsetzung)

\begin{tabular}{|c|c|c|c|c|c|c|c|c|c|c|}
\hline & \multicolumn{2}{|c|}{$\mathrm{t} 1$} & \multicolumn{2}{|c|}{$\mathrm{t} 2$} & \multirow[b]{2}{*}{$d$} & \multirow[b]{2}{*}{$p(T)$} & \multirow[b]{2}{*}{$r$} & \multirow[b]{2}{*}{$p(r)$} & \multirow[b]{2}{*}{$50 \%$} & \multirow[b]{2}{*}{$70 \%$} \\
\hline & $M$ & $S D$ & $M$ & $S D$ & & & & & & \\
\hline \multicolumn{11}{|c|}{ Optimismus gegenüber alltäglicher Anforderung ( $5 ; \alpha=.66)$} \\
\hline honest & 3.72 & 0.32 & 3.21 & 0.47 & -1.49 & $<.01$ & .27 & .11 & 36 & 17 \\
\hline wie-damals & 3.66 & 0.34 & 3.53 & 0.37 & -0.44 & .05 & .34 & .03 & 77 & 36 \\
\hline Faking-good & 3.62 & 0.33 & 3.92 & 0.18 & 1.18 & $<.01$ & .20 & .21 & 100 & 85 \\
\hline \multicolumn{11}{|c|}{ Rücksichtnahme bei sozialer Verantwortung $(5 ; \alpha=.66)$} \\
\hline honest & 3.72 & 0.33 & 3.58 & 0.36 & -0.68 & .01 & .57 & $<.01$ & 42 & 22 \\
\hline wie-damals & 3.62 & 0.38 & 3.53 & 0.43 & -0.38 & .13 & .69 & $<.01$ & 49 & 23 \\
\hline Faking-good & 3.65 & 0.32 & 3.75 & 0.40 & 0.35 & .06 & .50 & $<.01$ & 76 & 59 \\
\hline \multicolumn{11}{|c|}{ Selbstbehauptung bei Kommunikationserfordernis $(5 ; \alpha=.84)$} \\
\hline honest & 3.38 & 0.45 & 2.99 & 0.57 & -1.17 & $<.01$ & .58 & $<.01$ & 47 & 14 \\
\hline wie-damals & 3.35 & 0.54 & 3.09 & 0.61 & -0.63 & $<.01$ & .46 & $<.01$ & 51 & 31 \\
\hline Faking-good & 3.42 & 0.47 & 3.88 & 0.25 & 1.33 & $<.01$ & .19 & .24 & 98 & 93 \\
\hline \multicolumn{11}{|c|}{ Selbstvertrauen bei Prüfungsanforderung $(5 ; \alpha=.76)$} \\
\hline honest & 3.37 & 0.39 & 3.11 & 0.61 & -0.65 & $<.01$ & .50 & $<.01$ & 44 & 19 \\
\hline wie-damals & 3.33 & 0.50 & 3.30 & 0.51 & -0.09 & .72 & .54 & $<.01$ & 64 & 23 \\
\hline Faking-good & 3.26 & 0.54 & 3.90 & 0.20 & 1.73 & $<.01$ & .19 & .23 & 100 & 88 \\
\hline \multicolumn{11}{|c|}{ Stabilität bei stressvoller Anforderung $(5 ; \alpha=.77)$} \\
\hline honest & 3.28 & 0.44 & 2.56 & 0.62 & -1.87 & $<.01$ & .48 & $<.01$ & 39 & 25 \\
\hline wie-damals & 3.07 & 0.52 & 2.77 & 0.54 & -0.71 & $<.01$ & .28 & .09 & 56 & 28 \\
\hline Faking-good & 2.99 & 0.53 & 3.60 & 0.56 & 1.17 & $<.01$ & .24 & .14 & 93 & 90 \\
\hline \multicolumn{11}{|c|}{ Zufriedenheit mit dem Gesundheits- und Erholungsverhalten $(6 ; \alpha=.57)$} \\
\hline honest & 4.50 & 0.45 & 4.12 & 0.42 & -1.14 & $<.01$ & .43 & $<.01$ & 44 & 19 \\
\hline wie-damals & 4.44 & 0.60 & 4.29 & 0.63 & -0.42 & .06 & .60 & $<.01$ & 62 & 46 \\
\hline Faking-good & 4.30 & 0.49 & 4.80 & 0.31 & 1.47 & $<.01$ & .20 & .23 & 93 & 85 \\
\hline \multicolumn{11}{|c|}{ Zufriedenheit mit dem Leistungsverhalten $(6 ; \alpha=.69)$} \\
\hline honest & 4.22 & 0.49 & 3.80 & 0.49 & -1.35 & $<.01$ & .58 & $<.01$ & 42 & 22 \\
\hline wie-damals & 4.16 & 0.49 & 3.93 & 0.49 & -0.91 & $<.01$ & .74 & $<.01$ & 49 & 21 \\
\hline Faking-good & 4.03 & 0.50 & 4.69 & 0.45 & 1.62 & $<.01$ & .19 & .23 & 90 & 85 \\
\hline \multicolumn{11}{|c|}{ Zufriedenheit mit dem sozial-kommunikativen Verhalten $(3 ; \alpha=.69)$} \\
\hline honest & 4.30 & 0.36 & 3.98 & 0.40 & -1.12 & $<.01$ & .49 & $<.01$ & 47 & 28 \\
\hline wie-damals & 4.21 & 0.42 & 4.04 & 0.54 & -0.58 & .02 & .64 & $<.01$ & 51 & 36 \\
\hline Faking-good & 4.20 & 0.50 & 4.68 & 0.48 & 1.23 & $<.01$ & .28 & .08 & 90 & 85 \\
\hline
\end{tabular}

Anmerkung: Bei den drei Skalen zur Zufriedenheit lag die Spannweite zwischen 1 (sehr unzufrieden) und 5 (sehr zufrieden); bei allen übrigen Skalen zwischen 1 (stimmt gar nicht) und 4 (stimmt genau).

kommunikativer Situation wären mit einem Cut-off um $50 \%$ zu selektieren in der honest-Bedingung $44 \%$, in der wie-damals-Bedingung $44 \%$ und in der Faking-goodBedingung $73 \%$ aufgenommen worden. In allen anderen Skalen war dieser Prozentsatz deskriptiv betrachtet gleich hoch oder noch höher. Der höchste zeigte sich bei Opti- mismus gegenüber alltäglicher Anforderung und Selbstvertrauen bei Prüfungsanforderung, wo $100 \%$ der Faking-good-Bedingung aufgenommen worden wären. Bei einer Selektion von $70 \%$ wären (in den weiteren Skalen) 49-98 \% der Faking-good-Bedingung aufgenommen worden. Bei $50 \%$ Selektion lag der Prozentsatz der wie-da- 
Full Texts \& Dissemination

212 G. Krammer \& B. Pflanzl: Faking für Lehramtsstudienplätze

mals-Bedingung bis auf eine Ausnahme immer zwischen der honest- und Faking-good-Bedingung. Bei $70 \%$ Selektion lag die wie-damals-Bedingung sogar in 5 IPS-Skalen knapp unter der honest-Bedingung.

Im letzten Schritt wurde der Zusammenhang zwischen dem Faking und den Rangreihen analysiert. Zuerst wurden Rangkorrelationen zwischen t1 und t2 für jede IPS-Skala und Bedingung berechnet. Diese Rangkorrelationen sind in Tabelle 1 angeführt. Das Quadrat der Rangkorrelationen ist ein Effektstärkenmaß der Rangkorrelationen (Bortz \& Döring, 2006). In der honest-, wie-damals- bzw. Fakinggood-Bedingung zeigten sich in 14, 15 bzw. 3 IPS-Skalen signifikante Rangkorrelationen zwischen $\mathrm{t} 1$ und $\mathrm{t} 2$.

Anschließend wurde überprüft, ob es einen Zusammenhang zwischen dem mittleren Ausmaß an Faking und dem Erhalt der Rangreihe von t1 zu t2 gab: Die Rangkorrelationen wurden mit den Effektstärken des Vergleichs von $\mathrm{t} 1$ $\mathrm{zu} \mathrm{t} 2$ in den Bedingungen in Beziehung gesetzt. So zeigten sich z. B. in den drei Bedingungen von Aktivität in vertrauter kommunikativer Situation Effektstärken von $d=$ $-0.58,=-0.61$ bzw. $=0.57$ bei Rangkorrelationen von $r=$ $.45,=.69$ bzw. $=.13$. Diese mittleren Ausmaße an Faking (= Beträge der Effektstärken) wurden mit den Rangkorrelationen korreliert. Je höher das mittlere Ausmaß an Faking war, desto geringer fiel auch die Rangkorrelation aus $\left(r=-.43, p<.01, R^{2}=.19\right)$.

\section{Diskussion}

In der vorliegenden Studie wurde an einem Zulassungsverfahren für Lehramtsstudien der Einfluss von Faking auf Merkmalsausprägung und Rangreihe eines selbstbeschreibenden Persönlichkeitsinventars, das IPS, untersucht. Die Aufforderung, sich so geeignet wie möglich darzustellen, um ein Lehramtsstudium beginnen zu dürfen, bewirkte bis auf eine Ausnahme in allen IPS-Skalen, dass die Bewerbenden sich noch vorteilhafter als beim Zulassungsverfahren darstellten. Beim Zulassungsverfahren jedoch stellten sie sich bereits in allen bis auf zwei IPS-Skalen vorteilhafter dar, als sie es eigentlich waren. Diese vorteilhaftere Selbstdarstellung beim Zulassungsverfahren trat im Aus$\mathrm{maß}$ von $d=0.58$ bis $d=1.87$ auf, was ein höheres Maß an Verzerrung zeigt, als meta-analytisch für die Big Five in der Personalauswahl von Birkeland et al. (2006) berichtet wird $(0.19 \leq d \leq 0.45)$. In all diesen 16 IPS-Skalen konnten die Lehramtsstudierenden ihre Werte jedoch bedeutsam noch weiter ins Positive verzerren $(d=0.35$ bis $d=1.73)$.

Wenn Personen in Bewerbungssituationen zu Faking greifen, berücksichtigen sie, für welche Stelle (Birkeland et al., 2006) oder welches Studium (Ziegler, 2011) sie sich bewerben. Dies legt nahe, dass alle bis auf eine IPS-Skala - Rücksichtnahme bei sozialer Verantwortung - von den Lehramtsstudierenden als relevant für den Lehrerberuf angesehen wurden. Während dies für die Verwendung des IPS zur Auswahl künftiger Lehrkräfte spricht, spricht die auftretende Verzerrung gegen die Verwendung von Persön- lichkeitsinventaren bei Zulassungsverfahren für Lehramtsstudien. Wiederrum dafür spricht, dass weniger stark zu Faking gegriffen wurde, als es möglich gewesen wäre.

Eine Verzerrung der mittleren Merkmalsausprägung ist vor allem relevant, wenn Bewerbende für Lehramtsstudien auf der Basis von Cut-offs ausgewählt werden. Die vorliegende Studie unterstreicht, wie gravierend absichtliches Faking eine Studierendenauswahl auf der Basis von Cutoffs verzerren kann. Betrachtet man nur einzelne Skalen, können Personen sich so vorteilhaft darstellen, dass weitaus mehr Personen als intendiert aufgenommen werden würden. Es ist daher stark davon abzuraten, Studierende nur auf Basis von einzelnen Persönlichkeitsdimensionen zu selektieren.

Für eine faire Auswahl ist das größte Problem eine Verzerrung der Rangreihe durch Faking. In der vorliegenden Studie veränderten sich die Rangreihen umso mehr, je stärker Faking auftrat. Wenn Lehramtsstudierende sich absichtlich so geeignet wie möglich darstellten, gab es nur mehr in 3 von 18 IPS-Skalen einen Zusammenhang zwischen der Rangreihe dieser Darstellung und der des Zulassungsverfahrens. Diese Befunde decken sich mit den Ergebnissen von Griffith et al. (2007) zu Gewissenhaftigkeit unter denselben Bedingungen $(r=.08)$. Die Fairness der Zulassung zu einem Lehramtsstudium wäre daher nicht mehr gegeben, wenn Personen sich absichtlich so geeignet wie möglich darstellen. Jedoch zeigte sich in 14 von 18 der IPS-Skalen ein Zusammenhang der Rangreihen und einer ehrlichen Bearbeitung. Faking stellt damit auch bei Lehramtsstudien die Fairness von selbstbeschreibenden Verfahren in Zulassungsverfahren in Frage, jedoch scheint das natürlich auftretende Faking dies nur teilweise zu tun.

Fraglich ist, was in den beiden IPS-Skalen Durchsetzung in einer Führungsrolle und Aktives Erholungsverhalten in der Freizeit zwischen dem Zulassungsverfahren und der Nacherhebung geschah. In diesen beiden Skalen zeigten sich in der honest-Bedingung keine Unterschiede, während es in der Faking-good-Bedingung zu Unterschieden kam. Wahrscheinlich ist, dass sich die Wahrnehmung der Wichtigkeit dieser Merkmale verändert hat, z. B. durch die Ausbildung. Dies würde bedeuten, dass die Durchsetzung in einer Führungsrolle und das Aktive Erholungsverhalten in der Freizeit zum Zeitpunkt des Zulassungsverfahrens noch nicht als relevant für den Lehrerberuf erachtet wurden und sich im Laufe der Ausbildung diese Einschätzung veränderte. Es kann auch nicht ausgeschlossen werden, dass in der honest-Bedingung die Werte der Lehramtsstudierenden noch immer nicht die tatsächliche Merkmalsausprägung widerspiegelten. Die dadurch entstehende Unterschätzung des Fakings könnte diese Ergebnisse auch erklären.

Die Befunde zur Reproduzierbarkeit von Faking fielen nicht eindeutig aus. Eindeutig festgestellt werden konnte jedoch, dass Lehramtsstudierende ihr Faking beim Zulassungsverfahren nicht überschätzten. In allen IPS-Skalen, in denen das Faking nicht reproduziert werden konnte, lag eine Unterschätzung der Werte des Zulassungsverfahrens 
Full Texts \& Dissemination

G. Krammer \& B. Pflanzl: Faking für Lehramtsstudienplätze

vor. Diese Ergebnisse betonen den situativen Einfluss, der auch von Ellingson und McFarland (2011) postuliert wurde, und dass Faking ein sehr komplexer Prozess ist, dem viele Kognitionen zugrunde liegen (König et al., 2012).

$\mathrm{Zu}$ bedenken ist bei all diesen Befunden, dass Untersuchungen zu Zulassungsverfahren unter Varianzeinschränkung leiden (Linn, 1968). Für prädiktive Validitäten wirkt sich dies validitätsmindernd aus. Dadurch können die vorliegenden Zusammenhänge unterschätzt werden (Bortz \& Döring, 2006), da jene Personen nicht Teil dieser Studie sein konnten, welche nicht zugelassen wurden. Es muss offenbleiben, inwiefern die gezeigten Zusammenhänge und Nichtzusammenhänge Unterschätzungen waren.

Die vorliegende Studie ist ein erster Schritt, um Faking von Persönlichkeitsmerkmalen bei Bewerbenden für Lehramtsstudien zu untersuchen. Es wurde nur mit den Persönlichkeitsmerkmalen des IPS, einer gegebenen Selektionsquote und Studierenden in ihrem ersten Studienjahr beforscht. Die Ergebnisse können somit nicht darüber hinaus generalisiert werden. Es sollten in diesem Zusammenhang weitere Persönlichkeitsmerkmale untersucht werden. Außerdem sollte festgestellt werden, ob sich die vorliegenden Befunde bei anderen Selektionsquoten und höhersemestrigen Studierenden replizieren lassen. Da sich teilweise fragwürdige interne Konsistenzen zeigten, sollte für die gegebenen Persönlichkeitsmerkmale sichergestellt werden, dass sie in einem Zulassungsverfahren so gemessen werden, wie es intendiert ist. Es sollte auch an größeren Stichproben die Messinvarianz des Persönlichkeitsinventars überprüft werden. Weitere Studien sollten externe Kriterien wie bspw. objektiven und subjektiven Berufsoder Studienerfolg heranziehen, um zu untersuchen, ob und ggf. wie Faking den Zusammenhang des IPS in Bezug auf diese Kriterien beeinflusst. Auch bleiben die Fragen offen, wie sehr reproduziertes Faking diese Zusammenhänge beeinflusst und ob es Umstände gibt, unter denen tatsächliches Faking besser oder schlechter reproduziert werden kann.

\section{Elektronische Supplemente}

Die elektronischen Supplemente sind mit der OnlineVersion dieses Artikels verfügbar unter http://dx.doi. org/10.1024/1010-0652/a000161.

ESM 1. Tabelle.

Die Interkorrelationen der 18 IPS-Skalen zur Zulassung (t1) für die gesamte Stichprobe

ESM 2. Tabelle.

Die Interkorrelationen der 18 IPS-Skalen zur Zulassung (oberes Dreieck) und Nacherhebung (unteres Dreieck) für die honest-Bedingung samt interner Konsistenz $(\alpha)$ zur Nacherhebung

ESM 3. Tabelle.

Die Interkorrelationen der 18 IPS-Skalen zur Zulassung (oberes Dreieck) und Nacherhebung (unteres Dreieck) für die wie-damals-Bedingung samt interner Konsistenz $(\alpha)$ zur Nacherhebung

ESM 4. Tabelle.

Die Interkorrelationen der 18 IPS-Skalen zur Zulassung (oberes Dreieck) und Nacherhebung (unteres Dreieck) für die Faking-good-Bedingung samt interner Konsistenz $(\alpha)$ zur Nacherhebung

\section{Literatur}

Backhaus, K., Erichson, B., Plinke, W. \& Weiber, R. (2006). Multivariate Analysemethoden: eine anwendungsorientierte Einführung (13. Aufl.). Berlin: Springer-Lehrbuch.

Baumert, J. \& Kunter, M. (2011). Das mathematikspezifische Wissen von Lehrkräften, kognitive Aktivierung im Unterricht und Lernfortschritte von Schülerinnen und Schülern. In M. Kunter, J. Baumert, W. Blum, U. Klusmann, S. Krauss \& M. Neubrand (Hrsg.), Professionelle Kompetenz von Lehrkräften. Ergebnisse des Forschungsprogramms COACTIV (S. 163-192). Münster: Waxmann.

Birkeland, S. A., Manson, T. M., Kisamore, J., Brannick, M. T. \& Smith, M. A. (2006). A meta-analytic investigation of job applicant faking on personality measures. International Journal of Selection and Assessment, 14, 317-356.

Bortz, J. \& Döring, N. (2006). Forschungsmethoden und Evaluation für Human- und Sozialwissenschaftler (4., überarbeitete Auflage). Heidelberg: Springer.

Bortz, J. \& Schuster, C. (2010). Statistik für Human- und Sozialwissenschaftler (7. Auflage). Berlin: Springer-Verlag.

Bromme, R. \& Haag, L. (2004). Forschung zur Lehrerpersönlichkeit. In W. Helsper \& J. Böhme (Hrsg.), Handbuch der Schulforschung (S. 777-793). Wiesbaden: Verlag für Sozialwissenschaften.

Burns, G. N. \& Christiansen, N. D. (2011). Methods of measuring faking behavior. Human Performance, 24, 358-372.

Ellingson, J. E. \& McFarland, L. A. (2011). Understanding faking behavior through the lens of motivation: An application of VIE Theory. Human Performance, 24, 322-337.

Farsides, T. \& Woodfield, R. (2003). Individual differences and undergraduate academic success: The roles of personality, intelligence, and application. Personality and Individual Differences, $34,1225-1243$.

Field, A., Miles, J. \& Field, Z. (2012). Discovering Statistics Using R. USA: Sage Publications.

Griffin, B. \& Wilson, I. G. (2012). Faking good: Self-enhancement in medical school applications. Medical Education, 46, 485-490.

Griffith, R. L., Chmielowski, T. \& Yoshita, Y. (2007). Do applicants fake? An examination of the frequency of applicant faking behavior. Personnel Review, 36, 341-355.

Griffith, R. L. \& Peterson M. H. (2011). One piece at a time: The puzzle of applicant faking and a call for theory. Human Performance, 24, 291-301.

Hanfstingl, B. \& Mayr, J. (2007). Prognose der Bewährung im Lehrerstudium und im Lehrerberuf. Journal für LehrerInnenbildung, 7, 48-56.

Hell, B. \& Schuler, H. (2005). Verfahren der Studierendenauswah aus Sicht der Bewerber. Empirische Pädagogik, 19, 361-376.

Hell, B., Trapmann, S. \& Schuler, H. (2007). Eine Metaanalyse der Validität von fachspezifischen Studierfähigkeitstests im deutschsprachigen Raum. Empirische Pädagogik, 21, 251-270. 
Full Texts \& Dissemination

214 G. Krammer \& B. Pflanzl: Faking für Lehramtsstudienplätze

Hell, B., Trapmann, S., Weigand, S. \& Schuler, H. (2007). Die Validität von Auswahlgesprächen im Rahmen der Hochschulzulassung - eine Metaanalyse. Psychologische Rundschau $58,93-102$.

Judge, T. A., Higgins, C. A., Thoresen, C. J. \& Barrick, M. R. (1999). The Big Five personality traits, general mental ability, and career success across the life span. Personnel Psychology, $52,621-652$.

Kaub, K., Karbach, J., Biermann, A., Friedrich, A., Bedersdorfer, H., Spinath, F. M. et al. (2012). Berufliche Interessensorientierungen und kognitive Leistungsprofile von Lehramtsstudierenden mit unterschiedlichen Fachkombinationen. Zeitschrift für Pädagogische Psychologie, 26, 233-249.

Komar, S., Brown, D. J., Komar, J. A. \& Robie, C. (2008). Faking and the validity of conscientiousness: A Monte Carlo investigation. Journal of Applied Psychology, 93, 140-154.

König, C. J., Merz, A.-S. \& Trauffer, N. (2012). What is in applicants' minds when they fill out a personality test? Insights from a qualitative study. International Journal of Selection and Assessment, 20, 442-452.

Krammer, G. (2014, Dezember). General mental ability over and above domain-specific measures: Predicting academic achie vement in a 3 year longitudinal study on college admission. Paper presented at the Annual Conference of the International Society for Intelligence Research, Graz, AUT.

Kuncel, N. R. \& Hezlett, S. A. (2007). Standardized tests predict graduate student's success. Science, 315, 1080-1081.

Kuncel, N. R. \& Hezlett, S. A. (2010). Fact and fiction in cognitive ability testing for admissions and hiring decisions. Current Directions in Psychological Science, 19, 339-345.

Kuncel, N. R., Hezlett, S. A. \& Ones, D. S. (2001). A comprehensive meta-analysis of the predictive validity of the graduate record examinations: Implications for graduate student selection and performance. Psychological Bulletin, 127, 162-181.

Kuncel, N. R., Croded, M., Thomas, L., Klieger, D., Seiler, S. \& Woo, S. (2005). A meta-analysis of the validity of the Pharmacy College Admission Test (PCAT) and grade predictors of pharmacy student performance. American Journal of Pharmaceutical Education, 69, 339-347.

Levashina, J. \& Campion, M. A. (2006). A model of faking likelihood in the employment interview. International Journal of Selection and Assessment, 14, 299-316.

Levashina, J. \& Campion, M. A. (2007). Measuring faking in the employment interview: Development and validation of an in terview faking behavior scale. Journal of Applied Psychology, 92, 1638-1656.

Linn, R. L. (1968). Range restriction problems in the use of selfselected groups for test validiation. Psychological Bulletin, $69,69-73$.

Mayr, J. (2011). Der Persönlichkeitsansatz der Lehrerforschung. In E. Terhart, H. Bennewitz \& M. Rothland (Hrsg.), Handbuch der Forschung zum Lehrerberuf (S. 125-148). Münster Waxmann

Mayr, J. \& Brandstätter, H. (1998). Lehrer/in werden? Wien: Bundesministerium für Unterricht und kulturelle Angelegenheiten.

McFarland, L. A. \& Ryan, A. M. (2000). Variance in faking across noncognitive measures. Journal of Applied Psychology, $85,812-821$

Päßler, K. Hell, B. \& Schuler, H. (2011). Grundlagen der Berufsdiagnostik und ihre Anwendung auf den Lehrerberuf. Zeit schrift für Pädagogik, 57, 639-654.
Poropat, A. E. (2009). A meta-analysis of the Five-Factor Model of personality and academic performance. Psychological Bulletin, 135, 322-338.

Rosse, J. G., Stecher, M. D., Miller, J. L. \& Levin, R. A. (1998) The impact of response distortion on preemployment personality testing and hiring decisions. Journal of Applied Psychology, 83, 634-644.

Schaarschmidt, U. (2004). Halbtagsjobber. Weinheim: Beltz.

Schaarschmidt, U. \& Fischer, A. (2013). Manual Inventar zur Persönlichkeitsdiagnostik in Situationen (Version 21 - Revision 2). Mödling: SCHUHFRIED GmbH

Schmidt, F. L. \& Hunter, J. (2004). General mental ability in the world of work: Occupational attainment and job performance. Journal of Personality and Social Psychology, 86, 162-173.

Schulz-Kolland, R., Krammer, G., Rottensteiner, E. \& Weitlaner, R. (2014). Die Validität von Zulassungsverfahren - Befunde der Pädagogischen Hochschule Steiermark. Neuen @Hochschul-Zeitung, 3, 85-88.

Tett, R. P., Freund, K. A., Christiansen, N. D., Fox, K. E. \& Coaster, J. (2012). Faking on self-report emotional intelligence and personality tests: Effects of faking opportunity, cognitive ability, and job type. Personality and Individual Differences, 52, 195-201.

Trapmann, S., Hell, B., Hirn, J.-O. W. \& Schuler, H. (2007). Meta-analysis of the relationship between the Big Five and academic success at university. Zeitschrift für Psychologie, $215,131-151$

Unterbrink, T., Hack, A., Pfeifer, R., Buhl-Grießhaber, V., Müller, U., Wesche, H. et al. (2007). Burnout and effort-rewardimbalance in a sample of 949 German teachers. International Archives of Occupational and Environmental Health, 80, 433-441.

Viswesvaran, C. \& Ones, D. S. (1999). Meta-analyses of fakability estimates: Implications for personality measurement. Educational and Psychological Measurement, 59, 197-210.

Winkelspecht, C., Lewis, P. \& Thomas, A. (2006). Potential effects of faking on the NEO-PI-R: Willingness and ability to fake changes who gets hired in simulated selection decisions. Journal of Business and Psychology, 21, 243-259.

Ziegler, M. (2011). Applicant faking: A look into the black box. The Industrial-Organizational Psychologist, 49, 29-36.

Ziegler, M. \& Bühner, M. (2009). Modeling socially desirable responding and its effects. Educational and Psychological Measurement, 69, 548-565.

Ziegler, M., Danay, E., Schölmerich, F. \& Bühner, M. (2010). Predicting academic success with the Big 5 rated from different points of view: Self-rated, other rated and faked. European Journal of Personality, 24, 341-355.

Ziegler, M., Schmidt-Atzert, L., Bühner, M. \& Krumm, S. (2007). Fakability of different measurement methods for achievement motivation: Questionnaire, semi-projective, and objective. Psychology Science, 49, 291-307.

Georg Krammer

Pädagogische Hochschule Steiermark

Hasnerplatz 12

8010 Graz

Osterreich

georg.krammer@phst.at

Z. Pädagog. Psychol. 29 (3-4) @ 2015 Verlag Hans Huber, Hogrefe AG, Bern 
6.3 Study 3: The Psychometric Costs of Applicants' Faking: Examining Measurement Invariance and Re-test Correlations Across Response Conditions 
1

2

3 The Psychometric Costs of Applicants' Faking: Examining Measurement Invariance and Re-
Test Correlations Across Response Conditions

\section{Georg Krammer}

University College of Teacher Education Styria

Markus Sommer and Martin E. Arendasy

University of Graz
Please cite as:

Krammer, G., Sommer, M., \& Arendasy, M. E. (in press). The Psychometric Costs of Applicants' Faking: Examining Measurement Invariance and Re-Test Correlations Across Response Conditions. Journal of Personality Assessment.

Corresponding Author:

Georg Krammer

Hasnerplatz 12

8010 Graz, Austria

georg.krammer@phst.at 


\section{Abstract}

This study examines the stability of the response process and the rank-order of respondents responding to three personality scales in four different response conditions. Applicants to the University College of Teacher Education Styria ( $\mathrm{N}=243)$ completed personality scales as part of their college admission process. Half a year later, they retook the same personality scales in one of three randomly assigned experimental response conditions: honest, faking-good, or reproduce. Longitudinal means and covariance structure analyses showed that applicants' response processes could be partially reproduced after half a year, while respondents seemed to rely on an honest response behavior as a frame of reference. Additionally, applicants' faking behavior and instructed faking (faking-good) caused differences in the latent re-test correlations and consistently affected measurement properties. The varying latent re-test correlations indicated that faking can distort respondents' rank-order and thus the fairness of subsequent selection decisions, depending on the kind of faking behavior. Instructed faking (faking-good) even affected weak measurement invariance, while applicants' faking behavior did not. Consequently, correlations with personality scales - which can be utilized for predictive validity - may be readily interpreted for applicants. Faking behavior also introduced a uniform bias, implying that the classically observed mean raw score differences may not be readily interpreted.

Keywords: faking behavior, applicants, incumbents, faking-good, measurement invariance 


\section{Introduction}

Faking is a type of response bias wherein respondents distort their responses to personality scale items in order to be viewed more favorably (McFarland \& Ryan, 2000). Several studies indicated that faking can affect the psychometric properties of personality scales (e.g. Hartman \& Grubb, 2011; Miller \& Ruggs, 2014; Zickar \& Robie, 1999; Ziegler \& Bühner, 2009). Furthermore, selection decisions based on personality scales may be detrimentally affected due to applicants differing in their propensity and intensity to fake (e.g. Birkeland, Manson, Kisamore, Brannick \& Smith, 2006; Mueller-Hanson, Heggestad, \& Thornton, 2003; Rosse, Stecher, Miller \& Levin, 1998; Winkelspecht, Lewis \& Thomas, 2006; Zickar \& Robie, 1999). Therefore, it is important to examine how applicants fake personality scales in real life selection settings (Goffin \& Boyd, 2009; Griffith \& Peterson, 2011; Kuncel, Goldberg \& Kiger, 2011). To this end, the current study examined four different response behaviors to personality scales: (1) a real life admission testing situation, (2) a classic honest-condition, (3) an instructed faking-good-condition, and (4) a condition prompting incumbents to reproduce the response behavior from their admission testing setting. The comparison of the psychometric characteristics of the personality scales across these four response conditions is expected to shed some light on the processes used by applicants to fake personality scales in real life selection settings.

\section{Effects of Faking on Personality Scale Scores}

Practitioners are mainly concerned about the effects of faking on applicants' personality scale scores, and the effects on subsequent selection decisions. The high stakes involved in some personnel selection and educational admission settings are likely to prompt some sort of faking behavior from at least some applicants (cf. Dilchert, Ones, Viswesvaran \& Deller, 2006; Tett \& Simonet, 2011). Several laboratory studies using an instructed faking-goodcondition (i.e. instructions to "fake-good") indicate that respondents are capable of increasing their personality scales scores $(0.48 \leq d \leq 0.65$ in within-subject designs, and $0.47 \leq d \leq 0.93$ in between-subject designs: Viswesvaran \& Ones, 1999). However, some scholars argue that these detrimental effects of faking are specific to laboratory studies, and that in real life selection settings effects of faking on applicants' personality scale scores are negligible (e.g. Bradley \& Hauenstein, 2006; Hogan, Barrett \& Hogan, 2007; Ones, Dilchert, Viswesvaran \& Judge, 2007). This view has been partially supported by a recent meta-analysis indicating only negligible to small differences between applicants and incumbents $(0.11 \leq d \leq 0.45$; Birkeland et al., 2006). Unfortunately, the results of the meta-analysis cited above are 
Full Texts \& Dissemination

The Psychometric Costs of Applicants' Faking

1 necessarily based on the assumption that strong measurement invariance is given across

2 applicants and incumbents. If strong measurement invariance is given, respondents with the

3 same standing on the latent trait(s) have equal expected item and/or test scores. Thus, mean

4 differences within and between groups of respondents (e.g. applicants vs. incumbents) can be

5 interpreted at face value (e.g. Millsap, 2011; Mislevy et al., 2013). By contrast, if strong

6 measurement invariance is violated, mean score differences reflect not only (latent) true score

7 differences between the groups and/or conditions, but additionally measurement bias (cf. Li

8 \& Zumbo, 2009).

9 Studies examining re-test correlations of personality scales completed under different

10 response conditions offer some tentative evidence that faking may affect the psychometric

11 properties of personality scales. For example, Peterson, Griffith, Isaacson, O'Connell, and

12 Mangos (2011) re-tested applicants as incumbents, and reported a re-test correlation for 13 conscientiousness of $r=.62$. Similar results were obtained by Griffith, Chmielowski, and

14 Yoshita (2007), who reported a re-test correlation for conscientiousness of $r=.50$ for

15 applicants responding to a conscientiousness scale with and without motivation to fake. In

16 line with these findings, Hogan et al. (2007) reported re-test correlations for the Big Five

17 ranging from $r=.46$ to $r=.68$. In contrast to these findings, merely re-testing volunteers

18 generally yields higher re-test correlations (e.g. .70 $\leq r \leq .90$ : Arendasy, Sommer \&

19 Feldhammer, 2011;.67 $\leq r \leq .85$ : McCrae, Yik, Trapnell, Bond \& Paulhus, 1998; .50 $\leq r \leq$

20 .85: Hogan, 1992), as has already been pointed out by scholars (e.g. Hogan et al., 2007).

21 These lower than expected re-test correlations for applicants compared to volunteers may

22 indicate that faking compromises the measurement invariance of personality scales.

\section{Factors Affecting Faking on Personality Scale Items}

In order to interpret personality scale scores at face value, individual differences in the respondents' personality scale scores should be entirely attributable to individual differences in the respondents' standing on the latent trait measured (e.g. Millsap, 2011; Mislevy et al., 2013). This implies that respondents' standing on the latent trait should be the only individual differences construct having a causal effect on their responses to the personality scale items. However, several studies indicate that applicants consider a wide range of aspects when deciding which response category to endorse on personality scale items (e.g. Donovan, Dwight \& Hurtz, 2003; König, Merz \& Trauffer, 2012; Kuncel \& Tellegen, 2009; Ziegler, 2011). For instance, applicants evaluate the relevance of individual items for a particular position or education when considering faking their responses (cf. König et al., 2012; Ziegler, 
Full Texts \& Dissemination

The Psychometric Costs of Applicants' Faking

1 2011). Thus, their responses do not only depend anymore on their standing on the latent trait,

2 but also on their perceived relevance of the items, and their willingness to fake.

Several theoretical models have been proposed to explain why and how applicants fake in real life selection settings (e.g. Goffin \& Boyd, 2009; McFarland \& Ryan, 2000, 2006; Marcus, 2009; Mueller-Hanson, Heggestad, \& Thornton, 2006; Snell, Sydell \& Lueke, 1999). Despite various differences, all theoretical models cited above distinguish between dispositional and situational antecedents of applicants' faking behavior. Dispositional antecedents refer to individual differences, e.g. regarding honesty, or to appraisals of the relevance of personality traits for a particular job. On the other hand, situational antecedents

10 refer to more fluctuating characteristics of the assessment situation, such as the perceived 11 attractiveness of the job or the perceived competition level. In principle, these theoretical models can also be applied to an instructed faking-good-condition in a laboratory. For instance, when instructed to make a favorable impression for a particular job, one may expect that respondents consider the perceived relevance of personality scale items for this particular job. However, individual differences in the accuracy of the perceived relevance may be larger in the laboratory than in real life selection settings. By contrast, individual differences in respondents' willingness to fake and its antecedents may be less pronounced in laboratory settings than in a real life selection setting. This raises the question whether personality scales can be assumed to exhibit measurement invariance across various response conditions (faking-good, honest, applicants, etc.).

\section{Effects of Faking on Measurement Properties and Test Scores}

Researchers have found it useful to distinguish four levels of measurement invariance (cf. Cheung \& Rensvold, 2002; Millsap, 2011; Mislevy et al., 2013; Raykov, Marcoulides, \& Li, 2012; Vandenberg \& Lance, 2000). Each level builds on the prior level, and they are tested level by level. The lowest level is configural measurement invariance. The second level is weak measurement invariance. The third level is strong measurement invariance. The final level is strict measurement invariance. Faking behavior can theoretically affect personality scales on each level of measurement invariance, and personality test scores, which will be outlined below.

\section{Effects of faking on the levels of measurement invariance}

Configural measurement invariance only assumes the general factor structure of 34 personality scales to be equal across response conditions. Configural measurement invariance 
1 would be violated when a personality scale measures one latent trait in one response 2 condition, but more than one latent trait in another response condition. For example, consider 3 a questionnaire assessing the Big Five domain factor Extraversion. The questionnaire may 4 measure extraversion for incumbents. However, for applicants it may measure extraversion 5 and faking intensity. In this case, configural measurement invariance would be violated. 6 Consequently, scores of incumbents and applicants could not be directly compared in a 7 meaningful way (e.g. Cheung \& Rensvold, 2002; Millsap, 2011; Mislevy et al., 2013; 8 Vandenberg \& Lance, 2000).

Weak measurement invariance additionally assumes the items to be equally saturated 10 by the latent trait they have been intended to measure across response conditions. Weak 11 measurement invariance would be violated when an item reflects its latent trait to a different extent across response conditions. For example, consider the extraversion questionnaire again and an applicant for a teacher vacancy. Now, let one item be "I like to stay in contact with parents." Applicants may appraise this item as an indicator of their extraversion. For incumbents by contrast, this item content may be a necessary part of their job, and thus the item may be only moderately related to their extraversion. In this example, the item would reflect individual differences in extraversion for applicants, but would do so less in a sample of incumbents. The violation of weak measurement invariance also indicates that a nonuniform bias is present (for a detailed discussion of nonuniform bias cf. Barendse, Oort \& Garst, 2010; Penfield \& Camilli, 2007). Furthermore, weak measurement invariance across response conditions is necessary to compare correlations within one response condition to correlations within another response condition (Chen, 2008).

Strong measurement invariance additionally assumes the baseline probability of endorsing a specific response category to be equal across response conditions. Strong measurement invariance would be violated when respondents with the same standing on a latent trait have unequal item scores across different response conditions. Consider a respondent who moderately likes "staying in contact with parents". As an applicant for a teacher vacancy however, s/he may indicate to like "staying in contact with parents" to a high degree. With faking-good instructions for a teacher vacancy, s/he may even state to extremely like "staying in contact with parents". In this example, strong measurement invariance would be violated. This level of measurement invariance is necessary to interpret mean score differences at face value. If it is not given, a uniform bias is present (for a detailed discussion of uniform bias cf. Barendse, et al., 2010; Penfield \& Camilli, 2007). This implies that mean score differences do not only reflect mean individual differences, but also measurement bias 
(cf. Chen, 2008; Cheung \& Rensvold, 2002; Millsap, 2011; Li \& Zumbo, 2009).

Strict measurement invariance finally also assumes both the systematic variance not explained by the psychometric model, and the unsystematic variance of the measurement error to be equal across response conditions. Strict measurement invariance would be violated when responses are affected by their latent trait and situational factors. For example, applicants may also consider how badly they need a job when responding to items. This would introduce systematic variance in the item responses unaccounted for by the psychometric model. An instruction to fake-good could reverse this effect again, as all respondents might respond as if they desperately needed the job.

\section{Effects of faking on latent means and variances estimates}

Faking may affect the levels of measurement invariance, but also the latent means and/or variances of personality scales. When responding as applicants trying to qualify for a position, respondents may try to elevate their scores by endorsing higher response categories. If this is done equally for all items of a scale, then an increase in the latent mean will result. However, when raising scores, initially lower scores can be inflated by a greater extent. So respondents with a lower standing on the latent trait can raise their scores to a greater extent than respondents with an already higher standing on the latent trait (e.g. Goffin \& Boyd, 2009; McFarland \& Ryan, 2000, 2006). This in turn can lead to a decrease of the variance of the individual differences construct measured.

The extent to which faking affects the latent trait means and variances of a personality scale also depends on how homogeneously faking affects the items. Consider for example items of a personality scale differing in their perceived relevance for a position. If respondents choose to fake, the extent to which they will increase their scores will differ across these items. This in turn will lead to a violation of strong measurement invariance due to a uniform measurement bias (cf. Barendse, et al., 2010; Penfield \& Camilli, 2007). In contrast, if the responses to all items of one scale are elevated by the same extent, faking would increase the latent trait mean. Such a response behavior could not be distinguished from truly having a higher standing on the latent trait. Note that these two possible outcomes are not mutually exclusive. For instance, respondents may perceive all items of a personality scale as being relevant for a position, and still perceive selected items as being particularly relevant. In this case, the baseline probabilities of endorsing a specific response category would be globally higher, while being even higher for some items. This global increase would generalize to the latent mean, while the additional increase for selected items would 
1 violate strong measurement invariance. As a consequence, one would expect to observe a

2 shift of the latent mean, and a lack of strong measurement invariance.

In line with these considerations, studies examining the level of measurement invariance across honest- and faking-good-conditions yield results ranging from configural measurement invariance across these two conditions (e.g. Miller \& Ruggs, 2014), to weak measurement invariance (e.g. Zickar \& Robie, 1999), up to strong measurement invariance (Ferrando \& Anguiano-Carrasco, 2009a; 2009b). The inconsistent findings in these studies may be attributable to (1) the specific personality constructs measured and its perceived relevance for the position (e.g. psychoticism: Ferrando \& Anguiano-Carrasco, 2009a; impression management: Miller \& Ruggs, 2014), (2) the samples of respondents used in these studies (e.g. military: Zickar \& Robie, 1999; undergraduate business class students: Miller \& Ruggs, 2014), and/or (3) differences in test design characteristics such as item presentation mode (e.g. blocked: Ferrando \& Anguiano-Carrasco, 2009a; random: Zickar \& Robie, 1999).

Unfortunately, studies examining measurement invariance across real life selection settings and other response conditions are sparse. Robie, Zickar, and Schmit (2001) examined measurement invariance across applicants for a sales manager position and job incumbents.

The authors reported strict measurement invariance for five out of six personality scales examined. The one scale for which strict measurement invariance was violated exhibited a measurement bias for two items: one item favored applicants, while one item favored incumbents. Based on these mixed results, the authors argued for bias cancelation. Taken together, the currently available empirical evidence suggests that faking may affect the level of measurement invariance of personality scales. However, the extent to which this is the case seems to depend on the types of faking behavior compared (i.e. faking-good or applicants' faking), the position in question, and the characteristics of the personality scales used.

\section{Stability of Applicants' Faking Behavior}

Although several studies examined measurement invariance and mean raw score differences across various response conditions, little is known about the stability of individual differences in applicants' response behavior. Stability of response behavior has two important aspects, which need to be distinguished: (1) the stability of the response process, and (2) the stability of the rank-order of the respondents. The former can be addressed by examining measurement invariance, while the latter can be addressed by re-test correlations. Both aspects only partially build on each other. When configural or weak measurement invariance have been violated across response conditions, estimates of the re-test correlations within 
Full Texts \& Dissemination

The Psychometric Costs of Applicants' Faking

1 response conditions may be biased and therefore cannot be directly compared to each other.

2 By contrast, when weak measurement invariance across response conditions holds, re-test

3 correlations can be readily interpreted and compared across pairs of response conditions

4 (Chen, 2008). Unfortunately, neither weak nor strong measurement invariance guarantees

5 that the rank-order of respondents is preserved across response conditions. Measurement

6 invariance merely indicates the extent to which the response process can be assumed to be

7 invariant across response conditions (i.e. stability of the response process). For instance,

8 Zickar and Robbie (1999) outlined that faking may only result in a shift of the latent trait

9 without affecting strong or strict measurement invariance. According to this model,

10 applicants should fake personality scales in selection settings by imagining a more favorable

11 version of themselves. If this model has merit, one would expect the response process to the

12 personality scales to be identical across e.g. honest responding and real life selection settings.

13 However, since respondents differ in their propensity and intensity to fake (e.g. Birkeland et

14 al., 2006; Mueller-Hanson et al., 2003; Rosse et al., 1998; Winkelspecht et al., 2006; Zickar

15 \& Robie, 1999), the re-test correlations of personality scales across these two response

16 conditions may still be detrimentally affected. This may explain why re-testing applicants

17 generally yields smaller re-test correlations than re-testing volunteers (cf. Arendasy et al.,

18 2011; Griffin \& Wilson, 2012; Griffith et al., 2007; Hogan, 1992; Hogan et al., 2007; McCrae

19 et al., 1998; Peterson et al., 2011). This may also question the interpretation of test scores

20 obtained from incumbents who were instructed to respond like applicants (e.g. Fell \& König,

21 2016). Therefore, it is important to examine both aspects of stability separately.

22 Unfortunately, this has not been done so far.

\section{Description of the Current Study}

The present study examined the level of measurement invariance of three personality scales in a real life admission testing situation across four different response conditions. This was done in a combined within-subject and between-subject design. The research design is illustrated in Figure 1. At the first time point of measurement (selection, t1), respondents completed the personality scales as part of their admission testing process for studying at the University College of Teacher Education Styria. The personality scales of interest were one part of their admission process. Half a year later (re-test, $\mathrm{t} 2$ ), the personality scales were given for a second time to the applicants, who were by now incumbents. The incumbents were randomly assigned to one of the three response conditions, which only differed in the instructions of the personality scales. The first response condition was the honest-condition, 
Full Texts \& Dissemination

The Psychometric Costs of Applicants' Faking

1 for which incumbents were given the personality scales with the standard instructions. The 2 second response condition was the reproduce-condition, for which incumbents were 3 prompted to recall the situation of their selection process and to respond to the items as they 4 responded when they first took the admission test. The third response condition was the 5 faking-good-condition, for which incumbents were instructed to portray themselves as 6 favorably as possible in order to be admitted to the College University of Teacher Education 7 Styria. This specific research design was chosen because a combination of a real life selection 8 setting and a within-subject design has been argued to constitute a golden standard in faking 9 research (e.g. Komar, Brown, Komar \& Robie, 2008; Peterson et al., 2011; Ryan \& Boyce,

10 2006). Furthermore, the research design allowed us to examine both aspects of stability. < Insert Figure 1 here >

\section{Research Hypotheses}

For respondents in the honest-condition, we examined measurement invariance across the real life admission testing setting and the laboratory honest responses. Scholars (e.g. Bradley \& Hauenstein, 2006; Hogan et al, 2007; Ones et al., 2007) claimed that applicants' faking behavior only negligibly affects their personality test scores. In line with this hypothesis, strict measurement invariance was found across applicants and incumbents (Robie et al., 2001). Therefore, we hypothesized to find strict measurement invariance across applicants and incumbents instructed to respond honestly (hypothesis 1). However, previous studies indicated that faking distorts applicants' rank order (e.g. Mueller-Hanson et al., 2003; Rosse et al., 1998; Winkelspecht et al., 2006). Consistent with this hypothesis, moderate retest correlations were found between applicants and incumbents (e.g. Griffin \& Wilson, 2012; Griffith et al., 2007; Peterson et al., 2011). We therefore hypothesized to observe moderate re-test correlations between the real life admission testing setting and the honest responses (hypothesis 2). Lastly, samples of applicants have exhibited higher mean scores than samples of incumbents, although these effects were usually small (Birkeland et al., 2006). Therefore, we also hypothesized higher latent means of the applicants compared to the incumbents in our study (hypothesis 3 ).

The next condition compared applicants' response behavior in the real life admission testing situation to the response behavior in an instructed faking-good-condition. Instructed faking differs from applicants' faking, as all respondents can be assumed to fake irrespectively of individual differences in faking propensity (cf. Robie et al., 2001). Based on previous studies, we expected to observe at least configural measurement invariance (cf. 
1 Miller \& Ruggs, 2014). Since some studies (cf. Ferrando \& Anguiano-Carrasco, 2009a; 2 2009b) also observed strong measurement invariance, we hypothesized that it may even be 3 possible that strong measurement invariance holds across these two response conditions 4 (hypothesis 4). Furthermore, we hypothesized to find rather low re-test correlations between these two response conditions, because the lack of individual differences in faking propensity in the instructed faking-good-condition can be assumed to alter the rank-order of the 7 respondents (hypothesis 5). This prediction is also in line with previous studies examining the 8 re-test correlations between applicants and incumbents or volunteers instructed to fake-good (cf. Ellingson, Sackett, \& Hough, 1999; Griffith et al., 2007). For the same reason, instructed 10 faking-good should also lead to an increase in latent means compared to the applicants 11 (hypothesis 6).

The last condition examined the stability of applicants' response behavior across a real 13 life admission testing situation and the reproduced response behavior (reproduce-condition).

14 Based on the finding that the intensity and propensity of applicants' faking did not increase when rejected applicants re-applied for the same job (Hogan et al., 2007), we hypothesized applicants' faking behavior to be relatively stable. On the one hand, this should be reflected in the stability of the response process and would imply strict measurement invariance for incumbents attempting to reproduce their applicants' response behavior (hypothesis 7). On the other hand, the re-test correlation should also be high (hypothesis 8). Lastly, we expected consequence, latent mean difference across these two response conditions should be negligible (hypothesis 9).

\section{Method}

\section{Measures}

Studying applicants' faking behavior requires a personality inventory that is used for selection purposes. The Inventory for Personality Assessment in Situations (IPS: Schaarschmidt \& Fischer, 2013) is such a personality inventory, which is used for admission testing processes in teacher education (e.g. Schulz-Kolland, Krammer, Rottensteiner \& Weitlaner, 2014). The IPS is suitable for professions with high psychosocial demands, such as the teaching profession. Validation studies have shown that the IPS measures personality dispositions relevant to teaching training and to the teaching profession, and that there are characteristic personality profiles of both teachers and students in teacher education, which differ markedly from students of other courses of study (cf. Krammer, Sommer \& Arendasy, 
in press; Mayr \& Brandstätter, 1998; Schaarschmidt \& Fischer, 2013).

The IPS is comprised of three higher-order factors: social and communicative behavior, health and recreational behavior, and performance behavior. To study applicants' faking behavior, one subscale of each higher-order factor was chosen. Activity in familiar communicative situation was chosen for the higher-order factor social and communicative behavior, preventive health behavior in response to warning signals for the higher-order factor health and recreational behavior, and self-confidence in test situation for the higherorder factor performance behavior. Previous research demonstrates that activity in familiar communicative situation is highly related to the Big Five domain factor Extraversion, while self-confidence in test situation is highly related to the Big Five domain factor Emotional Stability/Neuroticism (cf. Koschmieder, Pretsch \& Neubauer, 2015). This may be due to the fact that the item contents of the scales represent sociableness and carefreeness respectively, which are both highly saturated by their respective domain factors Extraversion and Emotional Stability (Arendasy et al., 2011; Costa \& McCrae, 1992). Extraversion and Emotional Stability in turn have been shown to be relevant for success in teacher education and the teaching profession (e.g. Mayr, 2011). Lastly, preventive health behavior in response to warning signals is relevant for the teacher education and the teaching profession, as teachers are a high-risk group for burnout (e.g. Unterbrink et al., 2007), and the pressure of the profession can manifest itself already during the education (cf. Gold \& Roth, 1993).

All IPS scales consisted of a situational context and five personal behaviors that may occur in the described situation. Respondents had to indicate the extent to which they would exhibit each personal behavior in the described situation using a 4-point Likert scale (not true at all - definitely true). The situational context for activity in familiar communicative situation describes the setting of a sociable group of friends and acquaintances, and the items relate to the activity in this situation (e.g. "be very lively"). For the scale preventive health behavior in response to warning signals, the situational context is beginning to feel incapacitated, people already noticing this, and people suggesting a visit to the doctor. The items in this situation relate to the extent these warning signals are heeded, e.g. "actively do something for my health". Finally, the scale self-confidence in test situation describes a test situation and emphasizes the emotional burden of having to pass the test situation successfully. The items in this situation relate to one's own reaction to this pressure, e.g. "stay calm". To facilitate the interpretation, all items were recoded so that higher scores were more favorable. All three scales showed sufficient internal consistency at selection (t1: .639 $\leq$ $\alpha \leq .757)$. At re-test (t2), all three scaled exhibited good internal consistency in the honest- 
condition and reproduce-condition $(.720 \leq \alpha \leq .875)$, while the internal consistencies differed in the faking-good-condition ( $\alpha=.529, \alpha=.747$, and $\alpha=.862$, respectively).

\section{Procedure}

The research design is illustrated in Figure 1. The completion of personality scales for selection (t1) was a part of the admission testing process of the University College of Teacher Education Styria (for further details on the admission process: Schulz-Kolland et al., 2014). For the admission testing process, the applicants had to demonstrate adequate vocal function with no vocal chord disorders, followed by a test of their German skills, the measures for intelligence and personality, and finally an interview. All subtests of the admission testing process were relevant for admission. Applicants were ranked according to their final score, which was a weighted linear combination of all subtests. Applicants were aware that every part of the admission testing process would count for their ranking, but were unaware of the respective weights. Typically, approximately $60 \%$ of the applicants are admitted every academic year.

At re-test (t2), the applicants were re-tested with the same personality scales as incumbents. The incumbents were randomly assigned to the three experimental response conditions. In each experimental response condition (honest, reproduce, or faking-good) the personality scales were completed with differing instructions. Because of the instructions, respondents in the reproduce- and faking-good-condition were necessarily aware of the fact that the study was concerned with faking behavior. In contrast, respondents in the honestcondition were only given the standard instructions of the personality scales, to collect responses that were as honest as possible. These respondents were debriefed afterwards. The data of selection (t1) and of re-test (t2) was matched by a code, which could be generated out of the data at selection (t1) and which respondents were asked to generate themselves at retest (t2).

\section{Sample}

A total of $175(72.02 \%)$ females and $68(27.98 \%)$ males aged 18 to 44 years $(M=23.2$, $S D=4.8)$ participated in the present study. At re-test (t2), all respondents were in their second semester of a bachelor's degree to become either primary ( $\mathrm{n}=131 \sim 53.9 \%$ ) or secondary $(\mathrm{n}=112 \sim 46.1 \%)$ school teachers. Even though the respondents were randomly assigned to the three response conditions, the comparability of the three subsamples was examined. The respondents of the three response conditions $(n=81$ each) did not differ with 
Full Texts \& Dissemination

regard to gender $\left(\chi^{2}[2]=2.001, p=.368, V=.091\right)$, age $\left(F[2,240]=2.932, p=.055\right.$, partial $\eta^{2}$ $=.024)$, and their study majors $\left(\chi^{2}[2]=0.629, p=.730, V=.051\right)$. The data were collected

3 over a time span of two years. Therefore, the comparability across the two years of data 4 collection was examined. There were no differences in gender $\left(\chi^{2}[1]=0.067, p=.796, V=\right.$ $5.017)$, age $(t[241]=1.226, p=.222, d=0.158)$, and study major $\left(\chi^{2}[1]=0.141, p=.707, V=\right.$ 6 .024) across the two years. The data obtained in the first year of data collection had been used 7 in a previous study examining the prevalence of faking and its effect on the raw scores of 8 several personality scales (Krammer \& Pflanzl, 2015).

\section{Tested Models}

Measurement invariance, latent re-test correlations, and latent mean shifts were analyzed using multi-group means and covariance structure analysis. The multi-group means and covariance structure model is depicted in Figure 2. The three response conditions constituted the three groups. For each response condition, a one-factor model was specified for each measurement point. The latent factor at selection (t1) was hypothesized to correlate with the latent factor at re-test ( $\mathrm{t} 2$ ). The unstandardized factor loading of the first item was set to 1 for identification purposes at both measurement points. To account for the readministration of the same items, the residuals of the respectively same items were allowed to correlate. First, configural measurement invariance was tested, by specifying the described two-factor model in all three response conditions. Next, equality constraints were imposed to (1) test the comparability of the three response conditions at selection (t1) and to (2) test measurement invariance across the measurement points. The model fit was evaluated using the following goodness of fit statistics: non-significant $\chi^{2}$ statistic, RMSEA $<.06$, and CFI $\geq$ .95 (Hu \& Bentler, 1999; Marsh, Hau \& Wen, 2004).1 Each model fit was also compared to its less restrictive precursor using the following criteria: non-significant $\Delta \chi^{2}$ statistic and $\Delta \mathrm{CFI} \leq .002$ (Meade, Johnson \& Braddy, 2008). Since univariate (skewness and kurtosis $>|1|$ ) and multivariate (multivariate skewness > 26.668, all $p<.001$; MVN package: Korkmaz, Goksuluk \& Zararsiz, 2014) normality were violated, the parameters were estimated using a maximum likelihood estimator with robust standard errors and a SatorraBentler scaled test statistic (MLM: Satorra \& Bentler, 1994). This parameter estimation

1The widely used SRMR was omitted in our study. Studies suggest the SRMR is influenced by the sample size, and advise against using $\triangle$ SRMR for measurement invariance analysis (e.g. Meade et al., 2008). In accordance with these studies, Monte Carlo simulation studies on our final models suggested the SRMR to be heavily biased in evaluating our model fits, with the sample size causing Type I errors of $99 \%-100 \%$. A detailed summary of the Monte Carlo simulation studies is available from the first author upon request. 
1 method has been shown to perform well under similar conditions in simulation studies (e.g.

2 Curran, West \& Finch, 1996). For small sample sizes, this estimator also outperforms the

3 means and variance adjusted weighted least squares estimator (WLSMV) for measurement

4 invariance analysis (cf. Sass, Schmitt \& Marsh, 2014). All calculations were carried out in R

5 (R Core Team, 2015) using the lavaan package (Rosseel, 2012).

$<$ Insert Figure 2 here >

Ideally, the admission testing situation should exhibit strict measurement invariance, and equal latent means and variances across the three response conditions. To test this hypothesis, we first constrained the selection (t1) factor loadings to be equal across the three conditions $\left(\lambda_{t 1, \mathrm{~h}, 1}=\lambda_{t 1, \mathrm{r}, 1}=\lambda_{\mathrm{t} 1 \mathrm{f}, 1}\right.$, etc. $)$ to test for weak measurement invariance. Then, the selection (t1) item intercepts were constrained to be equal across the three conditions $\left(\tau_{\mathrm{t} 1, \mathrm{~h}, 1}=\right.$

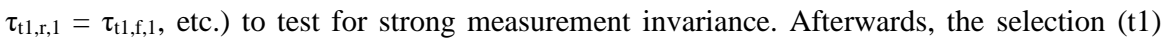
residual variances were constrained to be equal across the three conditions $\left(\theta \varepsilon_{t 1, \mathrm{~h}, 1}=\theta \varepsilon_{\mathrm{t} t \mathrm{r}, \mathrm{r}}=\right.$ $\theta \varepsilon_{t 1, f, 1}$, etc.) to test for strict measurement invariance. Finally, the latent trait variances and means were constrained to be equal across the three conditions $\left(\Phi_{\mathrm{t} 1, \mathrm{~h}}=\Phi_{\mathrm{t} 1, \mathrm{r}}=\Phi_{\mathrm{t} 1, \mathrm{f}}\right.$ and $\xi_{\mathrm{t} 1, \mathrm{~h}}=$ $\xi_{\mathrm{t} 1, \mathrm{r}}=\xi_{\mathrm{t} 1, \mathrm{f}}$, respectively).

After testing the comparability of the response conditions at selection (t1), measurement invariance across the measurement points was examined. Firstly, the factor loadings were constrained to be equal across the measurement points in each response condition to test for weak measurement invariance (i.e. $\lambda_{\mathrm{t} 1, \mathrm{~h}, 1}=\lambda_{\mathrm{t} 2 \mathrm{~h}, 1}, \lambda_{\mathrm{t} 1 \mathrm{~h}, 2}=\lambda_{\mathrm{t} 2 \mathrm{~h}, 2}$, etc.). Secondly, the item intercepts were constrained to be equal across the measurement points in each response conditions to test for strong measurement invariance (i.e. $\tau_{\mathrm{t} 1 \mathrm{~h}, 1}=\tau_{\mathrm{t} 2 \mathrm{~h}, 1}, \tau_{\mathrm{t} 1 \mathrm{~h}, 2}=$ $\tau_{\mathrm{t} 2 \mathrm{~h}, 2}$, etc.). Thirdly, the residual variances were constrained to be equal across the measurement points in each response conditions to test for strict measurement invariance (i.e. $\theta \varepsilon_{\mathrm{t} 1 \mathrm{~h}, 1}=\theta \varepsilon_{\mathrm{t} 2 \mathrm{~h}, 1}, \theta \varepsilon_{\mathrm{t} 1 \mathrm{~h}, 2}=\theta \varepsilon_{\mathrm{t} 2 \mathrm{~h}, 2}$, etc.). Afterwards, the equality of the residual covariances across the response conditions $\left(\mathrm{rc}_{1, \mathrm{~h}}=\mathrm{rc}_{1, \mathrm{r}}=\mathrm{rc}_{1, \mathrm{f}}\right.$, etc. $)$ was tested. Finally, the equality of the latent trait variances $\left(\Phi_{\mathrm{t} 1, \mathrm{~h}}=\Phi_{\mathrm{t} 2 \mathrm{~h}}\right.$, etc. $)$ and means $\left(\xi_{\mathrm{t} 1 \mathrm{~h}}=\xi_{\mathrm{t} 2 \mathrm{~h}}\right.$, etc. $)$ across the measurement points was tested.

If any of the above mentioned equality constraints failed to fit the data, constraints were successively relaxed, and the models again compared (cf. Meredith, 1993; Vandenberg \& Lance, 2000). This relaxation of parameters lead to partial invariance models. The partial invariance models were then compared to the last fitting model (e.g. partial strong measurement invariance model vs. weak measurement invariance model). In the case of multiple parameters differing (e.g. item intercepts in more than one response condition at 
1 selection (t2)), it was also tested whether additional equality constraints between these 2 parameters could be introduced (e.g. $\tau_{\mathrm{t} 2, \mathrm{~h}, 1}=\tau_{\mathrm{t} 2, \mathrm{r}, 1}$ ). This was repeated until equivalence was 3 achieved and the final models thereby specified. Within the final models, the equality of the

4 latent trait re-test correlations was then tested (i.e. $r_{h}=r_{r}=r_{f}$ ). 5

\section{Results}

\section{Comparison across Response Conditions at Selection (t1)}

First, measurement invariance at selection (t1) was examined for the comparability of the three response conditions at selection (t1). The global fit statistics and model comparisons 10 of the successively more restricted models are shown in the upper parts of Table 1 (activity in 11 familiar communicative situation), Table 2 (preventive health behavior in response to warning signals), and Table 3 (self-confidence in test situation). The results indicated that at selection (t1) strict measurement invariance across the three response conditions could be assumed for all scales (model \#1a - model \#4a, model \#1h - model \#4h, and model \#1s model \#4s, respectively). Furthermore, adding the constraints for equal latent trait variances (model \#5a, model \#5h, and model \#5s, respectively) and equal latent trait means (model \#6a, model \#6h, and model \#6s, respectively) did not worsen the model fit. Therefore, the respondents randomly assigned to the three response conditions at re-test (t2) did not differ in their response processes, latent trait mean, and latent trait variance at selection (t1) in all three scales.

\section{Comparison across Measurement Points}

Next, measurement invariance across selection ( $\mathrm{t} 1$ ) and re-test (t2) was tested to examine the extent to which faking behavior in the different response conditions affected the measurement properties of the scales. The global fit statistics of the successively more restricted models for testing measurement invariance across selection (t1) and re-test (t2), and the respective model comparisons are summarized in the lower parts of Table 1 (activity in familiar communicative situation), Table 2 (preventive health behavior in response to warning signals), and Table 3 (self-confidence in test situation). The final parameter estimates are summarized in Table 4, and the latent re-test correlations in Table 5.

\section{Activity in familiar communicative situation}

For the scales activity in familiar communicative situation (cf. Table 1), weak 34 measurement invariance (model \#7a) could be assumed across selection (t1) and re-test (t2). 
Full Texts \& Dissemination

The Psychometric Costs of Applicants' Faking

1 However, strong measurement invariance was not given (model \#8a): four intercepts differed

2 in the honest-condition and one in the faking-good-condition (model \#9a). The item

3 intercepts differed complementarily: they were either higher in the honest-condition at

4 selection (t1) than at re-test ( $\mathrm{t} 2$ ), or lower in the faking-good-conditions at selection (t1) than

5 at re-test ( $\mathrm{t} 2)$. This violation of strong measurement invariance in the honest-condition and

6 faking-good-condition was contrary to hypotheses 1 and 4, respectively. Next, the equality of

7 the residual variances was examined. This was the only scale where all residual variances

8 were equal across the measurement points (model \#10a). Thus, strict measurement invariance

9 was given in the reproduce-condition, which supported hypothesis 7. The residual

10 covariances were also equal across the response conditions (model \#11a).

Next, the equality of the latent trait variances, means, and co-variances was examined.

The latent trait variances were not equal across the measurement points (model \#12a); the latent trait variance in the honest-condition at selection (t2) was higher than all other latent trait variances (model \#13a). The latent trait means were also not equal (model \#14a), with the latent trait mean in the reproduce-condition at selection (t2) being lower than all other latent trait means (model \#15a). The latent trait mean difference in the reproduce-condition was contrary to hypothesis 9 , while the equal latent trait means in the honest-condition and faking-good-condition were contrary to hypotheses 3 and 6, respectively. Finally, the latent re-test correlations (cf. Table 5) differed across the three response conditions (model \#16a): the latent trait re-test correlation was highest in the reproduce-condition (supporting hypothesis 8 ), lower in the honest-condition (supporting hypothesis 2), and negligible in the faking-good-condition (supporting hypothesis 5). This was the only scale where no equality of the latent re-test correlations could be established (cf. Table 5).

\section{$<$ Insert Table 1 here >}

\section{Preventive health behavior in response to warning signals}

For the scale preventive health behavior in response to warning signals (cf. Table 2), weak measurement invariance was given across the measurement points (model \#7h), while strong measurement invariance was not (model \#8h). Two intercepts differed (model \#9h), one in the faking-good-condition ( $\mathrm{t} 1<\mathrm{t} 2$; not supporting hypothesis 4$)$, and one in the reproduce-condition ( $\mathrm{t} 1>\mathrm{t} 2$; not supporting hypothesis 7 ). Next, equality constraints on the residual variances were imposed (model $\# 10 \mathrm{~h}$ ), which showed that the residual variances differed across the measurement points. Overall, three residual variances were lower at selection (t1) than at re-test ( $\mathrm{t} 2)$; the residual variances of one item in the honest-condition 
Full Texts \& Dissemination

The Psychometric Costs of Applicants' Faking

1 and reproduce-condition to the same extend, and additionally another residual variance in the

2 honest-condition (model \#11h). These differences in the residual variances in the honest-

3 condition were contrary to hypotheses 1 . The covariances of the residuals were equal across

4 all three response conditions (model \#12h).

Afterwards, equality constraints for the latent trait variances, means, and co-variances were imposed. The latent trait variances were not equal across the measurement points (model \#13h), with the variance of the latent trait in the reproduce-condition at re-test (t2) being the highest (model \#14h). The latent trait means also differed across the measurement points (model \#15h): in the honest-condition and the reproduce-condition they were equally higher at selection ( $\mathrm{t} 1$ ) than at re-test (t2), while being lower in the faking-good-condition (model \#16h). The latent trait mean differences in the honest-condition and faking-goodcondition offered support for hypotheses 3 and 6, respectively. However, the latent trait mean difference in the reproduce-condition was contrary to hypothesis 9. Finally, the latent re-test correlations were also not equal across all three conditions (model \#17h), but were across the honest-condition and faking-good-condition (model \#18h). As can be seen in Table 5, the latent re-test correlations were moderate in the honest-condition and faking-good-condition (supporting hypothesis 2, while not supporting hypothesis 5, respectively), and higher in the reproduce-condition (supporting hypothesis 8 ).

\section{$<$ Insert Table 2 here >}

\section{Self-confidence in test situation}

The scale self-confidence in test situation (cf. Table 3) differed from the other two scales, as weak measurement invariance was not given (model \#7s). Subsequent analyses indicated that the factor loading of one item of this scale was higher in the faking-goodcondition than in all other response conditions (model \#8s). This violation of weak measurement invariance in the faking-good-condition contradicted hypothesis 4 . Similar to the other two scales, constraining the item intercepts to be equal across the measurement points decreased the model fit (model \#9s). One intercept differed per response condition (model \#10s): one intercept was equally higher in the honest-condition and reproducecondition at selection (t1) than at re-test ( $\mathrm{t} 2)$, while in the faking-good-condition one item intercept was lower at selection (t1) than at re-test (t2). The differences in intercepts in the honest-condition and reproduce-condition were contrary to hypotheses 1 and 7, respectively. The residual variances were also not equal across measurement points (model \#11s). No residual variances were affected in the honest-condition nor the reproduce-condition, while 
1 all residual variances in the faking-good-condition were higher at selection (t1) than at re-test

2 (t2). Regarding the residual covariances, they were equal across all three response conditions 3 (model \#13s).

Finally, the equality of the latent trait variances, means, and co-variances was examined. As with the other two scales, the latent trait variances were not equal across the measurement points (model \#14s). The latent trait variance was lower at selection (t1) than at 7 re-test (t2) in the honest-condition, while it was higher in the faking-good-condition (model 8 \#15s). The latent trait means also differed (model \#16s): the latent trait means in the honestcondition and reproduce-condition were lower at selection (t1) than at re-test (t2), while it

10 was higher at selection (t1) than at re-test (t2) in the faking-good-condition. Similar to the 11 results of the scale preventive health behavior in response to warning signals, these latent 12 trait mean differences supported hypotheses 3 and 6, while being contrary to hypothesis 9 . 13 This was the only scale were no further equality of the latent trait means could be established. 14 The latent re-test correlations were also unequal (model \#17s), but were equal across the 15 honest-condition and reproduce-condition (model \#18s). The latent re-test correlations (cf. 16 Table 5) were moderate in the honest- condition and reproduce-condition (supporting 17 hypothesis 2, while not supporting hypothesis 8 respectively), and lower in the faking-goodcondition (supporting hypothesis 5).

\section{$<$ Insert Table 3 here > \\ $<$ Insert Table 4 here > \\ $<$ Insert Table 5 here >}

\section{Discussion}

While it has been examined whether, and to what extent, applicants can fake personality scales, more research is needed to evaluate the effect of different kinds of faking behavior on the response process involved (Goffin \& Boyd, 2009; Griffith \& Peterson, 2011; Kuncel et al., 2011). The current study is expected to shed some light on applicants' faking behavior by re-testing applicants as incumbents in three response conditions: (1) honest responses, (2) faked-good responses, and (3) responses given when attempting to reproduce one's own responses from when applying. Measurement invariance, re-test correlations, and latent trait mean differences across these three response conditions and applicants' response behavior in a real life selection setting were examined. 


\section{Honest, Applicants', and Instructed Faking Responses}

It was hypothesized that the response behavior of applicants is comparable to the response behavior of incumbents completing the personality scale in an honest-condition (cf. Robie et al., 2001). The findings of the current study only partially support this hypothesis. None of the scales exhibited strict measurement invariance across applicants in a real life selection setting and incumbents responding honestly (contradictory to hypothesis 1). Faking in a real life selection setting caused respondents primarily to increase their responses to selected items. As this was not the case for all items to the same extent, strong measurement invariance was violated for two out of three scales. For the one scale where strong measurement invariance was given, strict measurement invariance was nevertheless violated. This decrease of unaccounted for variance from honest response behavior to applicants' response behavior seems to be attributable to real life selection settings reducing the potential ambiguity of item contents. If an item content is ambiguous, this would introduce variance unaccounted for by the psychometric model. However, being in a real life selection setting could reduce this ambiguity, because respondents will less likely relate the item content to all possible settings, but rather to the job relevant settings.

Nevertheless, our results corroborate that the detrimental effects of applicants' faking behavior should not be overestimated (e.g. Bradley \& Hauenstein, 2006; Hogan et al., 2007; Ones et al., 2007), as some measurement properties were consistently preserved (configural and weak measurement invariance). The preservation of weak measurement invariance also indicates that predictive validity coefficients may not be detrimentally affected (Chen, 2008). This may explain why the effects of applicants' faking behavior has been deemed to be negligible in real life selection settings based on a comparison of predictive validity coefficients across honest responding conditions and real life selection settings (for an overview cf. Ones et al., 2007).

The finding of a lack of strict measurement invariance across applicants' response behavior in a real life selection setting and the honest response behavior contradicts our hypothesis and the results of Robie et al. (2001) on which this hypothesis was based. However, there was one main difference between the present study and the study conducted by Robie et al. (2001). In the selection situation of Robie et al. (2001), a warning was presented "that distorted self-descriptions would invalidate the ... test results". No such warning has been presented in the current study. Prior research indicated that applicants wrongly fear their faking behavior may be exposed (König et al, 2012). Furthermore, it has been shown that a warning that faking can be detected reduces faking when the negative 
consequences of being detected are emphasized (e.g. Dwight \& Donovan, 2003; Goffin \& Woods, 1995). Therefore, applicants may have been more reluctant to fake in the study conducted by Robie et al. (2001) than in our present study.

In summary, the effects of applicants' faking behavior were not negligible in our study. Applicants' faking behavior compromised either strong or strict measurement invariance for all three scales. Furthermore, applicants' faking behavior distorted the rank-orders (hypothesis 2 ) to a modest extent ( $r=.497, r=.387$, and $r=.533$ ). This distortion was in line with previous research (e.g. Griffin \& Wilson, 2012; Griffith et al., 2007; Peterson et al., 2011) and our hypotheses (hypothesis 2), and corroborates the effect of detrimental applicants' faking behavior on selection decisions.

The response behavior in an instructed faking-good-condition was hypothesized to be different to other response behaviors, such as applicants' response behavior and honest response behavior (e.g. Miller \& Ruggs, 2014; Robie et al., 2001). The findings of the current study corroborate this hypothesis. This difference could be seen in the distortion of rankorder (hypothesis 5). This distortion was in line with previous studies (e.g. Ellingson, Sackett, \& Hough, 1999; Griffith et al., 2007), and showed that the rank-order could be completely distorted $(r=.019)$, but was at its highest still only modestly preserved $(r=.395)$. Furthermore, instructed faking compared to applicants' response behavior consistently violated strong measurement invariance (hypothesis 4). This was always the case for items that were not yet affected by applicants' faking behavior, underlining that applicants engage in less faking then they could.

For one scale, weak and strict measurement invariance were additionally violated across applicants' response behavior and instructed faking. This was the scale (selfconfidence in test situation) where instructed faking lead to a ceiling effect. Due to the limited variance in the items of this scale, the unaccounted variance of all items was also decreased. For the item with the most pronounced restriction of variance, even the factor loading was affected. This was also the item that had the highest average responses at selection (t1). The item content of this item was related to being stable, a trait for which even an overly high endorsement cannot be viewed negatively for teachers.

Taken together, our findings suggest that instructed faking will always detrimentally affect the measurement properties of scales. Moreover, this detrimental effect will be even more pronounced when instructed faking leads to strong ceiling effects. Consequently, caution is advised when making selection decisions: when faking behavior causes a ceiling effect - e.g. applicants are highly motivated to fake by very high selection ratios - the 
1 selection decisions may be heavily biased.

We hypothezised to find the lowest latent means for honest response behavior, higher latent means for applicants' response behavior, and even higher latent means for instructed faking-good response behavior (cf. Birkeland et al, 2006; Viswesvaran \& Ones, 1999). However, these hypotheses were only partially confirmed (hypotheses $3 \& 6$ ). The latent means of two of the three scales showed this expected pattern, while the latent means of the remaining scale (activity in familiar communicative situation) did not differ significantly across response conditions. Because of this, a post-hoc comparsion of this remaining scale's manifest raw means was done additionally. This post-hoc comparsion revealed the honest response behavior having a lower raw mean than the applicants' response behavior $(d=$ 0.36), which in turn had a lower raw mean then the instructed faking-good response behavior $(d=0.33)$. Like the aforementioned meta-analyses (Birkeland et al, 2006; Viswesvaran \& Ones, 1999), most studies on mean differences due to faking behavior only examine the mean difference of raw scores. Unfortunately, our finding that strong measurement invariance was not consistently given indicated that such mean raw score comparisons may be biased (Li \& Zumbo, 2009). If strong measurement invariance is unaffected or affected only partially however, the latent trait means will nevertheless show that mean honest responses are lower than mean applicants' responses, which are lower than mean intructed faking responses. Therefore, raw mean score differences due to faking behavior may reflect true differences, but, in the most detrimental case, may only reflect a uniform measurement bias.

\section{Stability of Applicants' Faking Behavior}

To try to shed even more light on applicants' faking behavior, the current study also addressed the stability of applicants' faking behavior over half a year. Based on previous findings, we hypothesized applicants' faking behavior to be relatively stable (cf. Hogan et al., 2007). Our results demonstrated that half a year later, incumbents could partially reproduce their response process from when they were applying (partial support for hypothesis 7). One scale exhibited strict measurement invariance (activity in familiar communicative situation), but for the other two scales, only partial strong measurement invariance was given. Additionally, an increase in unaccounted for variance by the psychometric model could be observed. For these affected items, the effects were consistent with an honest response behavior. Incumbents also consistently underestimated their faking intensity from when applying, i.e. their latent means decreased (contradictory to hypothesis 9). By contrast, the rank-orders (hypothesis 8 ) could remain remarkably stable $(r=.90$, and $r=.699$ ), but were at 
1 least equally high as when compared to honest response behavior $(r=.442)$. Consequently, 2 studies using test scores obtained from incumbents which were instructed to respond like 3 applicants may underestimate the effects of applicants' faking behavior (e.g. Fell \& König, 4 2016)

In summary, our results suggest that incumbents seem to be able to reproduce their response behavior from a real life selection setting, and when in doubt, they rely on an honest 7 responding behavior as a frame of reference. Given that half a year had passed between the 8 real life selection setting and the attempt to reproduce one's own faking behavior, it is remarkable that only a few measurement properties could not be reproduced. Therefore, these 10 findings suggest that the individual differences determinants of faking behavior may be 11 predominantly dispositional, as was already postulated by Snell et al. (1999). However, respondents underestimated their faking intensity when asked to reproduce their faking behavior from a real life selection setting. In consequence, situational factors may also play a non-negligible role.

\section{Limitations and Conclusion}

While the current study aimed to provide insights into applicants' response behavior, it is of course not without limitations. Firstly, only one personality inventory was used in the present study. Future research may consider using more than one personality inventory to examine the effects of faking in cases where scales differ in structure, response format, but also in their perceived relevance for the particular job. The perceived relevance of scales for a particular job may also be directly assessed in future studies, as the appraisal of relevance may differ between applicants and scholars. Also, more traditional Big Five inventories could be used, which do not relate the items to a situational context. However, it should be noted that the personality inventory used in this study behaved consistently with faking effects shown for more widely used personality inventories.

Future study may also wish to experimentally manipulate the selection ratio to examine the effects of the selection ratio on the propensity and intensity to fake. However, differences in selection ratios could affect the extent of range restrictions due to the selection process (Linn, 1968). This may lead to an underestimation of the correlations (Bortz \& Döring, 2006). To avoid such selection effects, future studies may also want to include rejected applicants in their samples. We do not believe that a range restriction significantly biased our results, since all three response conditions should be equally affected by this problem.

It would also be favorable to conduct future studies with larger sample sizes. The 
1 sample size in this study was rather small per group for measurement invariance analysis.

2 Due to this concern, subsequent Monte Carlo simulation studies were conducted to examine

3 if our parameter estimates were biased. Based on the final models, the Monte Carlo

4 simulation studies suggested that the parameter estimated were largely unbiased, and that

5 their standard errors were only slightly biased for two items of the scale activity in familiar

6 communicative situation. A detailed summary of the Monte Carlo simulation studies is

7 available from the first author upon request.

8 Furthermore, it remains unclear what caused respondents' underestimation of their own

9 faking intensity when attempting to reproduce their applicants' response behavior. Future

10 research should examine whether there are differences in the selection settings and individual

11 differences in the applicants by which the faking intensity can be reproduced with more or

12 with less accuracy. Finally, it can always be possible that responses in an honest-condition

13 may still not be entirely honest.

14 Despite these limitations, the current study improved our understanding of applicants'

15 response behavior in real life situations. The current study indicates that applicants' response

16 behavior in a real life situation is quite stable and faking primarily affects strong

17 measurement invariance by inducing a uniform bias as compared to an honest responding

18 behavior, which in turn detrimentally effects the stability of the rank-order of the respondents

19 according to their standing on the latent trait. However, it should be noted that these effects

20 are by far less severe than the effects of an instructed faking. 


\section{References}

Arendasy, M., Sommer, M., \& Feldhammer, M. (2011). Manual Big Five Structure Inventory (BFSI). Mödling: Schuhfried.

Barendse, M. T., Oort, F. J., \& Garst, G. J. A. (2010). Using restricted factor analysis with latent moderated structures to detect uniform and nonuniform measurement bias; a simulation study. AStA Advances in Statistical Analysis, 94, 117-127.

Birkeland, S. A., Manson, T. M., Kisamore, J., Brannick, M. T. \& Smith, M. A. (2006). A meta-analytic investigation of job applicant faking on personality measures. International Journal of Selection and Assessment, 14, 317-356.

Bortz, J., \& Döring, N. (2006). Forschungsmethoden und Evaluationfür Human- und Sozialwissenschaftler $\left(4^{\text {th }}\right.$ revised edition). Heidelberg: Springer.

Bradley, K. M., \& Hauenstein, N. M. (2006). The moderating effects of sample type as evidence of the effects of faking on personality scale correlations and factor structure. Psychology Science, 48, 313-335.

Chen, F. F. (2008). What happens if we compare chopsticks with forks? The impact of making inappropriate comparisons in cross-cultural research. Journal of Personality and Social Psychology, 95, 1005-1018.

Cheung, G. W., \& Rensvold, R. B. (2002). Evaluating Goodness of fit indexes for testing measurement invariance. Structural Equation Modelling, 9, 233-255.

Costa, P. T., \& McCrae, R. R. (1992). Neo PI-R professional manual. Odessa: Psychological Assessment Resources.

Curran, R. J., West, S. G., \& Finch, J. E. (1996). The robustness of test statistics to nonnormality and specification error in confirmatory factor analysis. Psychological Methods, 1, 16-29.

Dilchert, S., Ones, D. S., Viswesvaran, C., \& Deller, J. (2006). Response distortion in personality measurement: born to deceive, yet capable of providing valid self-assessments? Psychology Science, 48, 209-225.

Donovan, J. J., Dwight, S. A., \& Hurtz, G. M. (2003). An assessment of the prevalence, severity, and verifiability of entry-level applicant faking using the randomized response technique. Human Performance, 16, 81-106.

Dwight, S. A., \& Donovan, J. J. (2003). Do warnings not to fake reduce faking? Human Performance, 16, 1-23.

Ellingson, J. E., Sackett, P. R., \& Hough, L. M. (1999). Social desirability corrections in personality measurement: Issues of applicant comparison and construct validity. Journal of Applied Psychology, 84, $155-166$.

Fell, C. B., \& König, C. J. (2016). Cross-cultural differences in applicant faking on personality tests: a 43-nation study. Applied Psychology, 65, 671-717.

Ferrando, P. J., \& Anguiano-Carrasco, C. (2009a). Assessing the impact of faking on binary personality measures: An IRT-based multiple-group factor analytic procedure. Multivariate Behavioral Research, 44, 497-524.

Ferrando, P. J., \& Anguiano-Carrasco, C. (2009b). The interpretation of the EPQ Lie scale scores under honest and faking instructions: A multiple-group IRT-based analysis. Personality and Individual Differences, 46, $552-556$.

Goffin, R. D., \& Boyd, A. C. (2009). Faking and personality assessment in personnel selection: Advancing models of faking. Canadian Psychology, 50, 151-160.

Goffin, R. D., \& Woods, D. M. (1995). Using personality testing for personnel selection: Faking and test-taking inductions. International Journal of Selection and Assessment, 3, 227-236.

Gold, Y., \& Roth, R. A. (1993). Teachers managing stress and preventing burnout: The professional health solution. UK: Routledge.

Griffin, B., \& Wilson, I. G. (2012). Faking good: Self-enhancement in medical school applications. Medical Education, 46, 485-490.

Griffith, R. L., Chmielowski, T., \& Yoshita, Y. (2007). Do applicants fake? An examination of the frequency of applicant faking behavior. Personnel Review, 36, 341-355.

Griffith, R. L., \& Peterson M. H. (2011). One piece at a time: The puzzle of applicant faking and a call for theory. Human Performance, 24, 291-301.

Hartman, N. S., \& Grubb, W. L. (2011). Deliberate faking on personality and emotional intelligence measures. Psychological Reports, 108, 120-138.

Hogan, R. (1992). Hogan Personality Inventory Manual (Third Edition). Hogan Assessment Systems: Tulsa, Ok.

Hogan, J., Barrett, P., \& Hogan, R. (2007). Personality Measurement, Faking, and Employment Selection. Journal of Applied Psychology, 92, 1270-1285.

Hu, L., \& Bentler, P. M. (1999). Cutoff criteria for fit indexes in covariance structure analysis: Conventional criteria versus new alternatives. Structural Equation Modeling, 6, 1-55.

Kaplan, D. (2000). Structural equation modeling: Foundations and extensions. Thousand Oaks, CA: Sage. 
Komar, S., Brown, D. J., Komar, J. A., \& Robie, C. (2008). Faking and the validity of conscientiousness: A Monte Carlo investigation. Journal of Applied Psychology, 93, 140-154.

König, C. J., Merz, A.-S., \& Trauffer, N. (2012). What is in applicants' minds when they fill out a personality test? Insights from a qualitative study. International Journal of Selection and Assessment, 20, 442-452.

Korkmaz, S., Goksuluk, D., \& Zararsiz, G. (2014). MVN: An R Package for Assessing Multivariate Normality. The R Journal, 6, 151-162.

Koschmieder, C., Pretsch, J., \& Neubauer, A. (2015). Emotional Intelligence, Personality and General Mental Ability in teacher student selection: An Examination of Predictive Validity and Overlap. Paper presented at the Conference of the International Society for the Study of Individual Differences (ISSID) in London, CAN, July 2015.

Krammer, G., \& Pflanzl, B. (2015). Faking von Persönlichkeitseigenschaften bei Zulassungsverfahren für Lehramtsstudien. Zeitschrift für Pädagogische Psychologie, 29, 205-214.

Krammer, G., Sommer, M., \& Arendasy, M. E. (in press). Realistic Job Expectations Predict Academic Achievement. Learning and Individual Differences.

Kuncel, N. R., Goldberg, L. R., \& Kiger, T. (2011). A Plea for Process in Personality Prevarication. Human Performance, 24, 373-378.

Kuncel, N. R., \& Tellegen, A. (2009). A conceptual and empirical reexamination of the measurement of the social desirability of items: Implications for detecting desirable response style and scale development. Personnel Psychology, 62, 201-228.

Li, Z., \& Zumbo, B. D. (2009). Impact of differential item functioning on subsequent statistical conclusions. Psicológica, 30, 343-370.

Linn, R. L. (1968). Range restriction problems in the use of self-selected groups for test validiation. Psychological Bulletin, 69, 69-73.

Marcus, B. (2009). 'Faking' from the applicant's perspective: A theory of self-presentation in personnel selection settings. International Journal of Selection and Assessment, 17, 417-430.

Marsh, H. W., Hau, K. T., \& Wen, Z. (2004). In search of golden rules: Comment on hypothesis testing approaches to setting cut off values for fit indexes and dangers in overgeneralizing Hu \& Bentler's (1999) findings. Structural Equation Modeling, 11, 320-341.

Mayr, J. (2011). Der Persönlichkeitsansatz der Lehrerforschung. In E. Terhart, H. Bennewitz \& M. Rothland (Hrsg.), Handbuch der Forschung zum Lehrerberuf (S. 125-148). Münster: Waxmann.

Mayr, J. \& Brandstätter, H. (1998). Lehrer/in werden? Wien: Bundesministerium für Unterricht und kulturelle Angelegenheiten.

McCrae, R. R., Yik, M. S., Trapnell, P. D., Bond, M. H., \& Paulhus, D. L. (1998). Interpreting personality profiles across cultures: bilingual, acculturation, and peer rating studies of Chinese undergraduates. Journal of Personality and Social Psychology, 74, 1041

McFarland, L. A., \& Ryan, A. M. (2000). Variance in faking across noncognitive measures. Journal of Applied Psychology, 85, 812-821.

McFarland, L. A., \& Ryan, A. M. (2006). Toward an integrated model of applicant faking behavior. Journal of Applied Social Psychology, 36, 979-1016.

McFarland, L. A., Ryan, A. M., \& Ellis, A. (2002). Item placement on a personality measure: Effects on faking behavior and test measurement properties. Journal of Personality Assessment, 78, 348-369.

Meade, A. W., Johnson, E. C., \& Braddy, P. W. (2008). Power and sensitivity of alternative fit indices in tests of measurement invariance. The Journal of Applied Psychology, 93, 568-592.

Meredith, W. (1993). Measurement invariance, factor-analysis and factorial invariance. Psychometrika, 58, 525543.

Miller, B. K., \& Ruggs, E. N. (2014). Measurement invariance tests of the Impression Management sub-scale of the Balanced Inventory of Desirable Responding. Personality and Individual Differences, 63, 36-40.

Millsap, R. E. (2011). Statistical approaches to measurement invariance. New York: Routledge.

Mislevy, R. J., Haertel, G., Cheng, B. H., Ructtinger, L., DeBarger, A., Murray, E., Rose, D., Gravel, J., Colker, A. M., Rutstein, D., \& Vendlinski, T. (2013). A "conditional" sense of fairness in assessment. Educational Research and Evaluation: An International Journal on Theory and Practice, 19, 121-140.

Mueller-Hanson, R. A., Heggestad, E. D., \& Thornton III, G. C. (2003). Faking and selection: Considering the use of personality from select-in and select-out perspectives. Journal of Applied Psychology, 88, 348-355.

Mueller-Hanson, R. A., Heggestad, E. D., \& Thornton III, G. C. (2006). Individual differences in Impression Management: An exploration of the psychological processes underlying faking. Psychology Science, 48, 288-312.

Ones, D. S., Dilchert, S., Viswesvaran, C., \& Judge, T. A. (2007). In support of personality assessment in organizational settings. Personnel psychology, 60, 995-1027.

Penfield, R. D., \& Camilli, G. (2007). Differential item functioning and item bias. In C. R. Rao, \& S. Sinharay (Eds.). Handbook of statistics (pp. 125-167) North Holland: Elsevier. 
Peterson, M. H., Griffith, R. L., Isaacson, J. A., O'Connell, M. S., \& Mangos, P. M. (2011). Applicant faking, social desirability, and the prediction of counterproductive work behaviors. Human Performance, 24, 270290.

Raykov, T., Marcoulides, G. A., \& Li, C. H. (2012). Measurement invariance for latent constructs in multiple populations: A critical view and refocus. Educational and Psychological Measurement, 72, 954-974.

$\mathrm{R}$ Core Team. (2015). R: A language and environment for statistical computing [Computer software manual]. Vienna, Austria. Retrieved from http://www.R-project.org/

Robie, C., Zickar, M. J., \& Schmit, M. J. (2001). Measurement equivalence between applicant and incumbent groups: An IRT analysis of personality scales. Human Performance, 14, 187-207.

Rosse, J. G., Stecher, M. D., Miller, J. L., \& Levin, R. A. (1998). The impact of response distortion on preemployment personality testing and hiring decisions. Journal of Applied Psychology, 83, 634-644.

Rosseel, Y. (2012). lavaan: An R Package for Structural Equation Modeling. Journal of Statistical Software, 48, 1-36.

Ryan, A. M., \& Boyce, A. S. (2006). What do we know and where do we go? Practical directions for faking research. In R. L. Griffith \& M. H. Peterson (Eds.). A closer examination of applicant faking behavior (pp. 357-371). Greenwich, CT: Information Age.

Sass, D. A., Schmitt, T. a., \& Marsh, H. W. (2014). Evaluating Model Fit With Ordered Categorical Data Within a Measurement Invariance Framework: A Comparison of Estimators. Structural Equation Modeling: A Multidisciplinary Journal, 21, 167-180.

Satorra, A., \& Bentler, P. M. (1994). Corrections to test statistics and standard errors in covariance structure analysis. In A. von Eye \& C. C. Clogg (Eds.), Latent variables analysis: Applications for developmental research (pp. 399-419). Thousand Oaks, CA: Sage.

Schaarschmidt, U., \& Fischer, A. (2013). Manual Inventar zur Persönlichkeitsdiagnostik in Situationen (Version 21 - Revision 2). Mödling: SCHUHFRIED GmbH.

Schulz-Kolland, R., Krammer, G., Rottensteiner, E., \& Weitlaner, R (2014). Die Validität von Zulassungsverfahren - Befunde der Pädagogischen Hochschule Steiermark. Neuen @Hochschul-Zeitung, 3, $85-88$

Snell, A. F., Sydell, E. J., \& Lueke, S. B. (1999). Towards a theory of applicant faking: Integrating studies of deception. Human Resource Management Review, 9, 219-242.

Tett, R. P., \& Simonet, D. V. (2011). Faking in personality assessment: A “multisaturation" perspective on faking as performance. Human Performance, 24, 302-321.

Unterbrink, T., Hack, A., Pfeifer, R., Buhl-Grießhaber, V., Müller, U., Wesche, H., Frommhold, M., Scheuch, K., Seibt, R., Wirsching, M.\& Bauer, J. (2007). Burnout and effort-rewardimbalance in a sample of 949 German teachers. International Archives of Occupational and Environmental Health, 80, 433-441.

Vandenberg, R. J., \& Lance, C. E. (2000). A review and synthesis of the measurement invariance literature: Suggestions, practices, and recommendations for organizational research. Organizational Research Methods, 3, 4-70.

Viswesvaran, C., \& Ones, D. S. (1999). Meta-analyses of fakability estimates: Implications for personality measurement. Educational and Psychological Measurement, 59, 197-210.

Winkelspecht, C., Lewis, P., \& Thomas, A. (2006). Potential effects of faking on the NEO-PI-R: Willingness and ability to fake changes who gets hired in simulated selection decisions. Journal of Business and Psychology, 21, 243-259.

Zickar, M. J., \& Robie, C. (1999). Modeling faking good on personality items: An item-level analysis. Journal of Applied Psychology, 84, 551-563.

Ziegler, M. (2011). Applicant faking: A look into the black box. The Industrial-Organizational Psychologist, 49, 29-36.

Ziegler, M., \& Bühner, M. (2009). Modeling socially desirable responding and its effects. Educational and Psychological Measurement, 69, 548-565. 


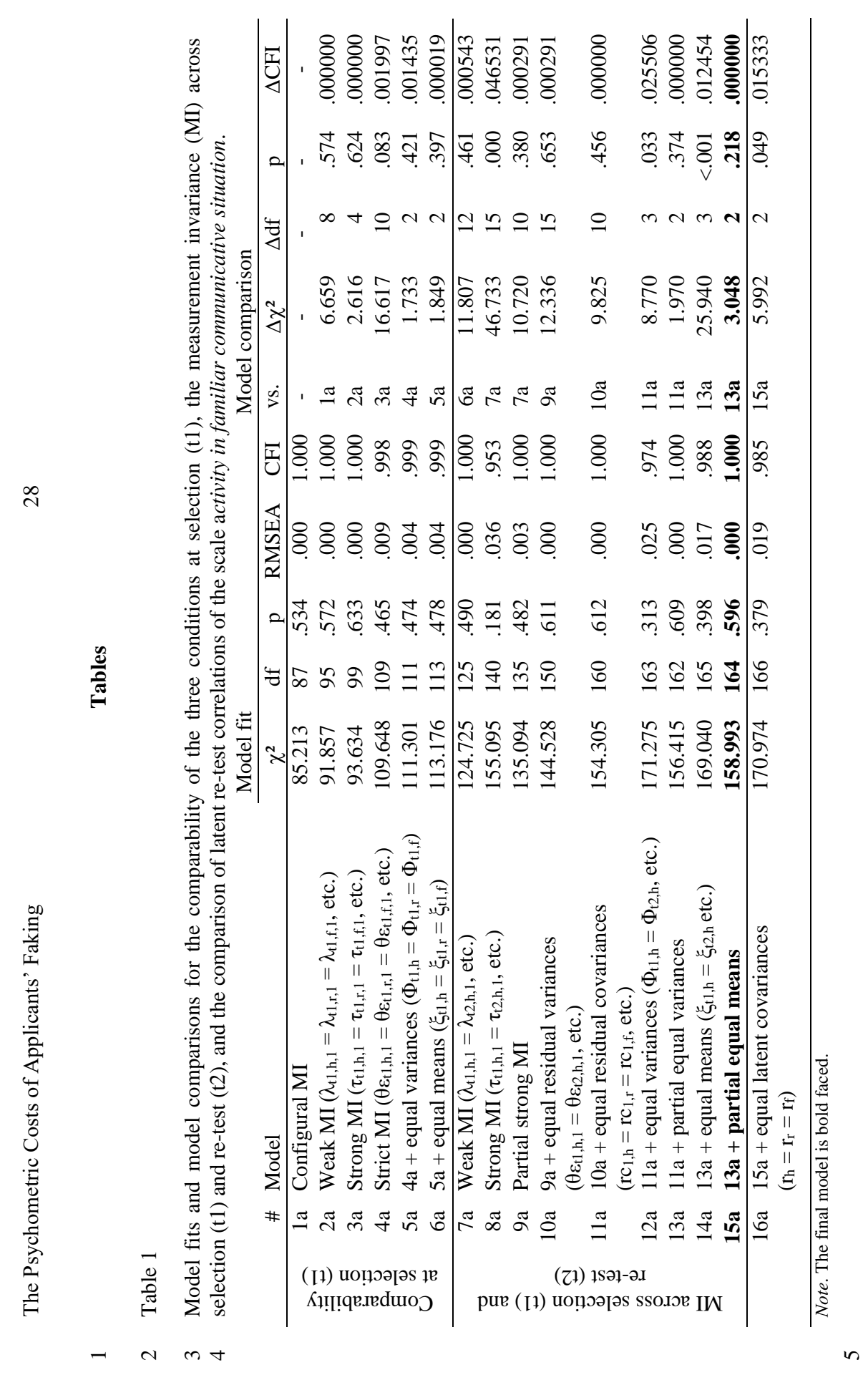




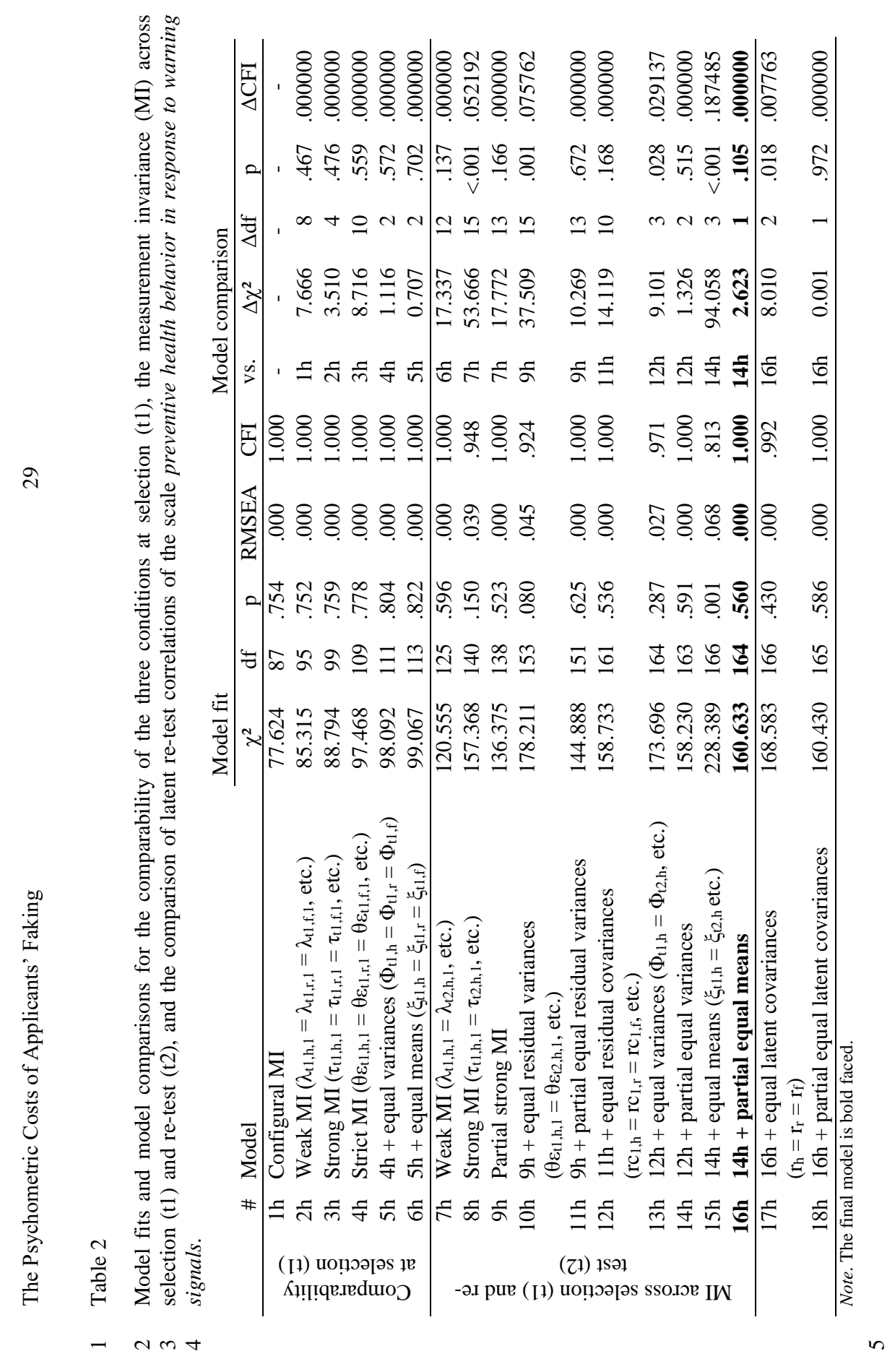




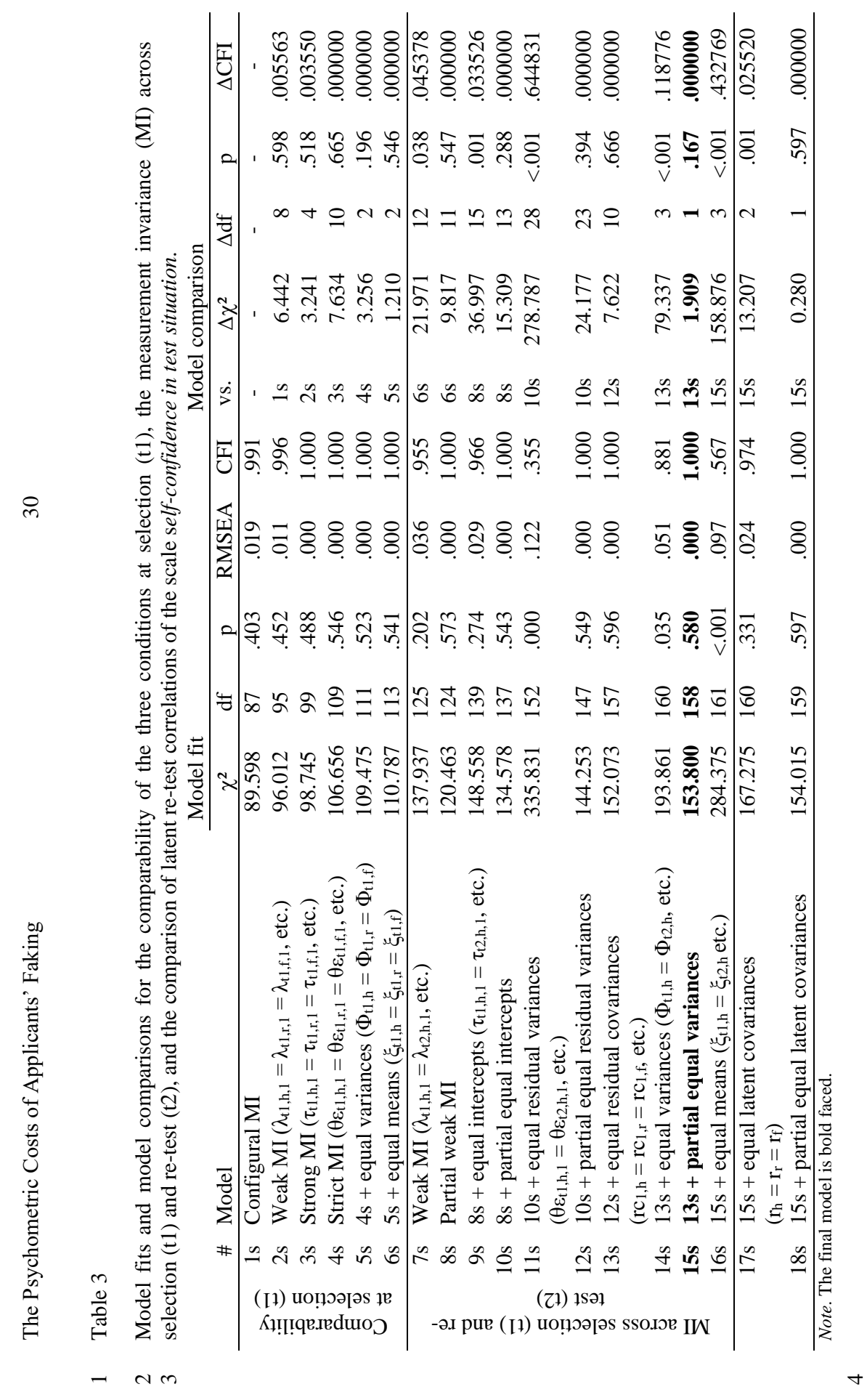




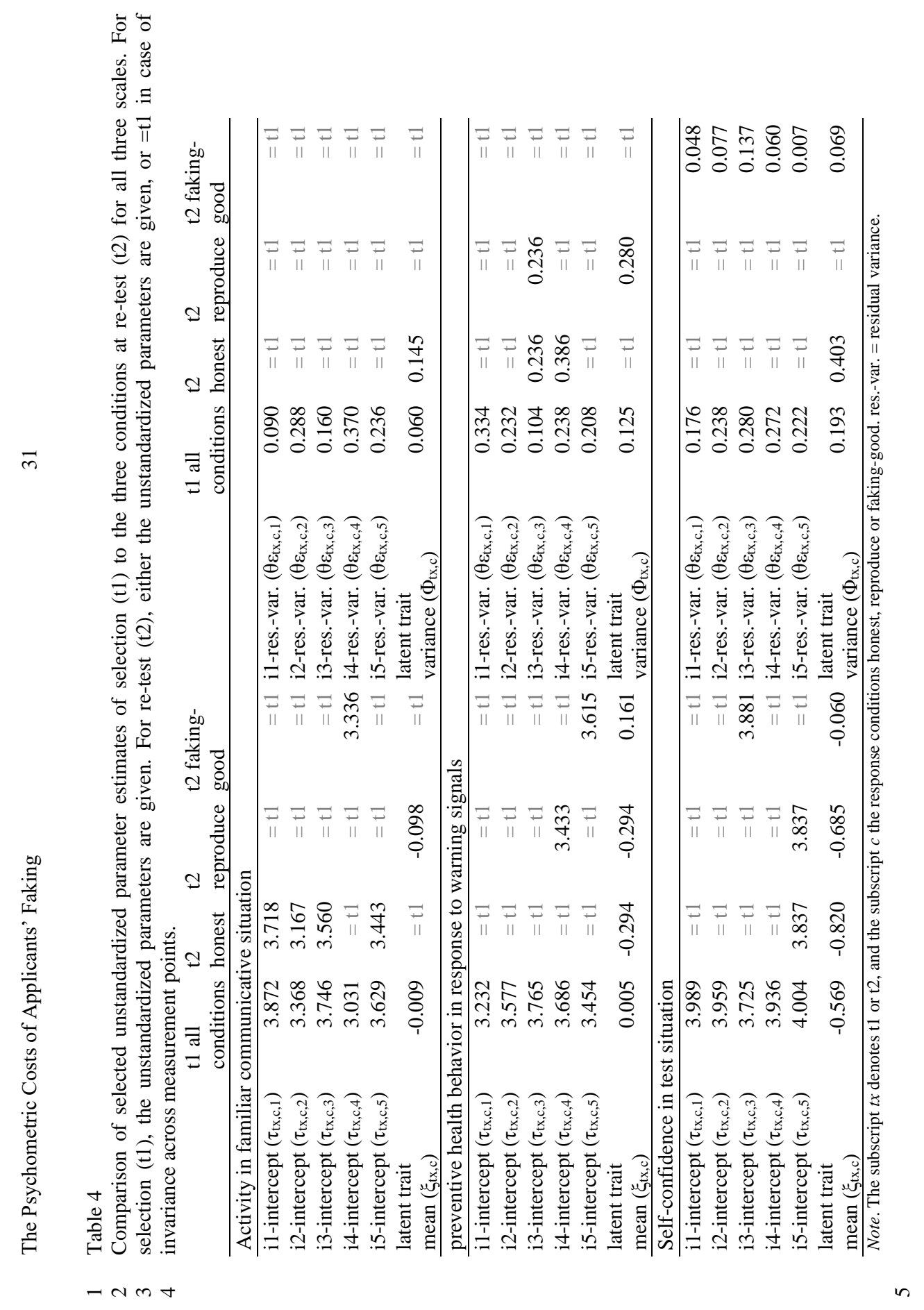




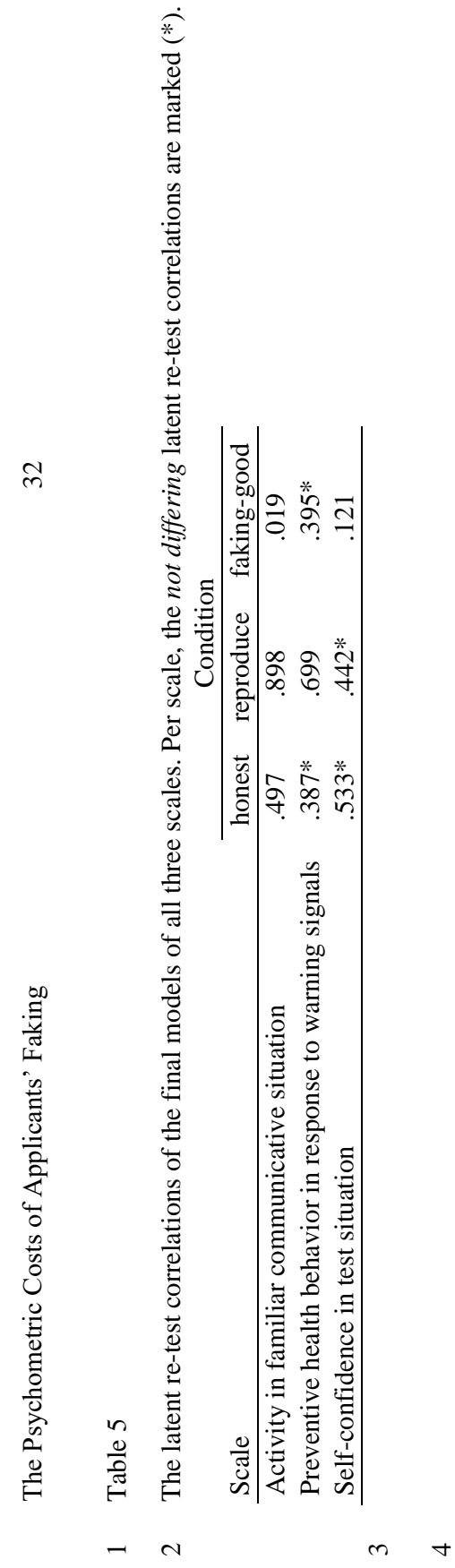




\section{Figures}

Figure 1 (cf. Figure 2 in Section 3.1):

The combined within-subject and between-subject design. At selection (t1), all respondents were applicants and went through the same admission testing situation. At re-test (t2), the same applicants were by now incumbents, and were randomly assigned to one of three response conditions.

Figure 2 (cf. Figure 3 in Section 4.1):

The multi-group means and covariance structure model, containing onefactor models at selection (t1) and at re-test (t2), and the covariances. The $\lambda_{\mathrm{s}}$ denote the factor loadings, the $\tau \mathrm{s}$ the intercepts, the $\theta \epsilon \mathrm{s}$ the residual variances, the $r c \mathrm{~s}$ the residual covariances, the $\xi \mathrm{s}$ the latent trait means, the $\Phi \mathrm{s}$ the latent trait variances, and $r$ s the latent trait covariance. 


\section{$7 \quad$ References}

Ackerman, P. L., \& Heggestad, E. D. (1997). Intelligence, Personality, and Interests: Evidence for Overlapping Traits. Psychological Bulletin, 121 (2), 219-245. doi: 10.1037/0033-2909.121.2.219

Arendasy, M., Hornke, L., Sommer, M., Häusler, J., Wagner-Menghin, M., Gittler, G., ... Körtner, T. (2012). Intelligence-Structure-Battery (INSBAT). Mödling: Schuhfried.

Barendse, M. T., Oort, F. J., \& Garst, G. J. A. (2010). Using restricted factor analysis with latent moderated structures to detect uniform and nonuniform measurement bias; a simulation study. AStA Advances in Statistical Analysis, 94(2), 117-127. doi: 10.1007/s10182-010-0126-1

Birkeland, S. A., Manson, T. M., Kisamore, J. L., Brannick, M. T., \& Smith, M. A. (2006). A meta-analytic investigation of job applicant faking on personality measures. International Journal of Selection and Assessment, 14(4), 317-335. doi: 10.1111/j.1468-2389.2006.00354.x

Bradley, K. M., \& Hauenstein, N. M. A. (2006). The moderating effects of sample type as evidence of the effects of faking on personality scale correlations and factor structure. Psychology Science, 48(3), 313.

Braxton, J. M., Vesper, N., \& Hossler, D. (1995). Expectations for College and Student Persistence. Research in Higher Education, 36(5), 595612. doi: $10.1007 / \mathrm{BF} 02208833$

Chen, F. F. (2008). Sensitivity of Goodness of Fit Indexes to Lack of Measurement Invariance. Structural Equation Modeling, 14(3), 464-504. doi: $10.1080 / 10705510701301834$

Cheung, G. W., \& Rensvold, R. B. (2002). Evaluating Goodness-of- Fit Indexes for Testing Measurement Invariance. Structural Equation Modeling: A Multidisciplinary Journal, 9(2), 233-255. doi: 10.1207/ S15328007SEM0902

Cooke, D. K., Sims, R. L., \& Peyrefitte, J. (1995). The Relationship between graduate student attitudes and attrition. Journal of Psychology, 129(6), 677-688. doi: 10.1080/00223980.1995.9914938

Credé, M., \& Kuncel, N. R. (2008). Study Habits, Skills, and Attitudes. Perspectives on Psychological Science, 3(6), 425-453. doi: 10.1111/ j.1745-6924.2008.00089.x

D'Agostino, J. V., \& Powers, S. J. (2009). Predicting Teacher Performance With Test Scores and Grade Point Average: A Meta-Analysis. American Educational Research Journal, 46(1), 146-182. doi: 10.3102/ 0002831208323280

Darling-Hammond, L., \& Lieberman, A. (2012). Teacher education around the world: Changing policies and practices. New York: Routledge. 
Dilchert, S., Ones, D. S., Viswesvaran, C., \& Deller, J. (2006). Response distortion in personality measurement: born to deceive, yet capable of providing valid self-assessments? Psychology Science, 48(3), 209-225.

Dwight, S. A., \& Donovan, J. J. (2003). Do Warnings Not to Fake Reduce Faking? Human Performance, 16(1), 1-23. doi: 10.1207/ S15327043HUP1601_1

Earnest, D. R., \& Dwyer, W. O. (2010). In their Own Words: an Online Strategy for Increasing Stress-Coping Skills among College Freshmen. College Student Journal, $44(4), 888-900$.

Farsides, T., \& Woodfield, R. (2003). Individual differences and undergraduate academic success: The roles of personality, intelligence, and application. Personality and Individual Differences, 34 (7), 1225-1243. doi: 10.1016/S0191-8869(02)00111-3

Geving, A. M. (2007). Identifying the types of student and teacher behaviours associated with teacher stress. Teaching and Teacher Education, 23(5), 624-640. doi: 10.1016/j.tate.2007.02.006

Goffin, R. D., \& Boyd, A. C. (2009). Faking and personality assessment in personnel selection: Advancing models of faking. Canadian Psychology/Psychologie canadienne, 50(3), 151-160. doi: 10.1037/a0015946

Goffin, R. D., \& Woods, D. M. (1995). Using personality testing for personnel selection: Faking and test-taking inductions. International Journal of Selection and Assessment, 3(4), 227-236. doi: 10.1111/j.1468-2389 .1995.tb00035.x

Gold, Y., \& Roth, R. A. (1993). Teachers managing stress and preventing burnout: The professional health solution (Routledge, Ed.). UK.

Griffin, B., \& Wilson, I. G. (2012). Faking good: Self-enhancement in medical school applicants. Medical Education, 46(5), 485-490. doi: 10.1111/j.1365-2923.2011.04208.x

Griffith, R. L., Chmielowski, T., \& Yoshita, Y. (2007). Do applicants fake? An examination of the frequency of applicant faking behavior. Personnel Review, 36 (3), 341-355. doi: 10.1108/00483480710731310

Gustafsson, J.-E., \& Balke, G. (1993). General and specific abilities as predictors of reading achievement. Multivariate Behavioral Research, 28(4), 407-434. doi: 10.1207/s15327906mbr2804_2

Heene, M., Hilbert, S., Draxler, C., Ziegler, M., \& Bühner, M. (2011). Masking misfit in confirmatory factor analysis by increasing unique variances: A cautionary note on the usefulness of cutoff values of fit indices. Psychological Methods, 16(3), 319-336. doi: 10.1037/a0024917

Hell, B., Trapmann, S., \& Schuler, H. (2007). Eine Metaanalyse der Validität von fachspezifischen Studierfähigkeitstests im deutschsprachigen Raum [A metaanalytic investigation of discipline-specific admission tests in 
German-speaking countries]. Empirische Pädagogik, 21(3), 251-270.

Hembree, R. (1988). Correlates, Causes, Effects, and Treatment of Test Anxiety. Review of Educational Research, 58(1), 47-77. doi: 10.3102/ 00346543058001047

Hogan, J., Barrett, P., \& Hogan, R. (2007). Personality measurement, faking, and employment selection. Journal of Applied Psychology, 92(5), 12701285. doi: 10.1037/0021-9010.92.5.1270

Komar, S., Brown, D. J., Komar, J. a., \& Robie, C. (2008). Faking and the validity of conscientiousness: a Monte Carlo investigation. The Journal of applied psychology, 93(1), 140-54. doi: 10.1037/0021-9010.93.1.140

König, C. J., Merz, A. S., \& Trauffer, N. (2012). What is in Applicants' Minds When They Fill Out a Personality Test? Insights from a qualitative study. International Journal of Selection and Assessment, 20(4), 442452. doi: $10.1111 /$ ijsa. 12007

Krammer, G., \& Pflanzl, B. (2015). Faking von Persönlichkeitseigenschaften bei Zulassungsverfahren für Lehramtsstudien. Zeitschrift für Pädagogische Psychologie, 29(3-4), 205-214. doi: 10.1024/1010-0652/ a000161

Krammer, G., Sommer, M., \& Arendasy, M. E. (2016). Realistic job expectations predict academic achievement. Learning and Individual Differences, 51, 341-348. doi: 10.1016/j.lindif.2016.09.010

Krammer, G., Sommer, M., \& Arendasy, M. E. (in press). The psychometric costs of applicants' faking: examining measurement invariance and retest correlations across response conditions. Journal of Personality Assessment.

Kuncel, N. R., \& Hezlett, S. a. (2007). Standardized Tests Predict Graduate Students' Success. Science, 315(5815), 1080-1081. doi: 10.1126/ science. 1136618

Kuncel, N. R., \& Hezlett, S. a. (2010). Fact and Fiction in Cognitive Ability Testing for Admissions and Hiring Decisions. Current Directions in Psychological Science, 19(6), 339-345. doi: 10.1177/ 0963721410389459

Kuncel, N. R., Hezlett, S. A., \& Ones, D. S. (2004). Academic Performance, Career Potential, Creativity, and Job Performance: Can One Construct Predict Them All? Journal of Personality and Social Psychology, 86(1), 148-161. doi: 10.1037/0022-3514.86.1.148

Kuncel, N. R., Hezlett, Sarah, A., \& Ones, D. S. (2001). A comprehensive meta-analysis of the predictive validity of the Graduate Record Examinations: Implications for graduate student selection and performance. Psychological Bulletin, 127(1), 162-181.

Kuncel, N. R., Wee, S., Serafin, L., \& Hezlett, S. a. (2010). The Validity 
of the Graduate Record Examination for Master's and Doctoral Programs: A Meta-Analytic Investigation. Educational and Psychological Measurement, 70 (2), 340-352. doi: 10.1177/0013164409344508

Lei, P. W. (2009). Evaluating estimation methods for ordinal data in structural equation modeling. Quality \&3 Quantity, 43(3), 495-507. doi: $10.1007 / \mathrm{s} 11135-007-9133-\mathrm{Z}$

Li, Z., \& Zumbo, B. D. (2009). Impact of differential item functioning on subsequent statistical conclusions based on observed test score data. Psicologica, 33(2), 343-370. doi: 10.14288/1.0054576

Marcus, B. (2009). 'Faking' From the Applicant's Perspective: A theory of selfpresentation in personnel selection settings. International Journal of Selection and Assessment, 17(4), 416-430. doi: 10.1111/j.1468-2389 .2009.00483.x

Mayr, J. (2011). Der Persönlichkeitsansatz der Lehrerforschung. In H. Bennewitz \& M. Rothland (Eds.), Handbuch der forschung zum lehrerberuf (pp. 125-148). Münster: Waxmann.

McFarland, L. A., \& Ryan, A. M. (2000). Variance in faking across noncognitive measures. Journal of Applied Psychology, 85(5), 812-821.

McFarland, L. A., \& Ryan, A. M. (2006). Toward an integrated model of applicant faking behavior. Journal of Applied Social Psychology, 36 (4), 979-1016. doi: 10.1111/j.0021-9029.2006.00052.x

Meade, A. W., Johnson, E. C., \& Braddy, P. W. (2008). Power and sensitivity of alternative fit indices in tests of measurement invariance. The Journal of Applied Psychology, 93(3), 568-592. doi: 10.5465/AMBPP.2006.27182124

Millsap, R. E. (2011). Statistical approaches to measurement invariance. New York: Routledge.

Mislevy, R. J., Haertel, G., Cheng, B. H., Ructtinger, L., DeBarger, A., Murray, E., .. Vendlinski, T. (2013). A "conditional" sense of fairness in assessment. Educational Research and Evaluation, 19(2-3), 121-140. doi: 10.1080/13803611.2013.767614

Mueller, R. O., \& Hancock, G. R. (2008). Best practices in structural equation modeling. In J. W. Osborne (Ed.), Best practices in quantitative methods (pp. 488-508). Thousand Oaks, CA: Sage.

Mueller-Hanson, R., Heggestad, E. D., \& Thornton, G. C. (2003). Faking and selection: considering the use of personality from select-in and selectout perspectives. The Journal of applied psychology, 88(2), 348-355. doi: 10.1037/0021-9010.88.2.348

Mueller-Hanson, R., Heggestad, E. D., \& Thornton, G. C. (2006). Individual Differences in Impression Management: An Exploration of the Psychological Processes Underlying Faking. Psychology Science, 48(3), 
$288-312$.

O'Conner, M. C., \& Paunonen, S. V. (2007). Big Five personality predictors of post-secondary academic performance. Personality and Individual Differences, 43(5), 971-990. doi: 10.1016/j.paid.2007.03.017

Ones, D. S., Dilchert, S., Viswesvaran, C., \& Judge, T. A. (2007). in Support of Personality Assessment in Organizational Settings. Personnel Psychology, 60(60), 995-1027. doi: 10.1111/j.1744-6570.2007.00099.x

Paunonen, S. V., \& Ashton, M. C. (2001). Big Five factors and facets and the prediction of behavior. Journal of Personality and Social Psychology, 81(3), 524-539. doi: 10.1037/0022-3514.81.3.524

Penfield, R. D., \& Camilli, G. (2007). Differential item functioning and item bias. In C. R. Rao \& S. Sinharay (Eds.), Handbook of statistics (pp. 125-167). North Holland: Elsevier.

Peterson, M. H., Griffith, R. L., Isaacson, J. a., O'Connell, M. S., \& Mangos, P. M. (2011). Applicant Faking, Social Desirability, and the Prediction of Counterproductive Work Behaviors. Human Performance, $24(3)$, 270-290. doi: 10.1080/08959285.2011.580808

Poropat, A. E. (2009). A meta-analysis of the five-factor model of personality and academic performance. Psychological Bulletin, 135(2), 322-338. doi: $10.1037 / a 0014996$

Raykov, T., Marcoulides, G. A., \& Li, C.-H. (2012). Measurement Invariance for Latent Constructs in Multiple Populations: A Critical View and Refocus. Educational and Psychological Measurement, 72(6), 954-974. doi: $10.1177 / 0013164412441607$

Reeve, C. L. (2004). Differential ability antecedents of general and specific dimensions of declarative knowledge: More than g. Intelligence, 32(6), 621-652. doi: 10.1016/j.intell.2004.07.006

Richardson, M., Abraham, C., \& Bond, R. (2012). Psychological correlates of university students' academic performance: a systematic review and meta-analysis. Psychological Bulletin, 138(2), 353-87. doi: 10.1037/ a0026838

Robie, C., Zickar, M. J., \& Schmit, M. J. (2001). Measurement Equivalence Between Applicant and Incumbent Groups: An IRT Analysis of Personality Scales. Human Performance, 14(2), 187-207. doi: 10.1207/S15327043HUP1402_04

Ronfeldt, M. (2012). Where should student teachers learn to teach? Effects of field placement school characteristics on teacher retention and effectiveness. Educational Evaluation and Policy Analysis, 34(1), 3-26. doi: 10.3102/0162373711420865

Rosse, J. G., Stecher, M. D., Miller, J. L., \& Levin, R. a. (1998). The impact of response distortion on preemployment personality testing 
and hiring decisions. Journal of Applied Psychology, 83(4), 634-644. doi: 10.1037/0021-9010.83.4.634

Rosseel, Y. (2012). lavaan: An R Package for Structural Equation Modelin. Journal of Statistical Software, 48(2), 1-36. doi: 10.18637/jss.v048.i02

Ryan, A. M., \& Boyce, A. S. (2006). What do we know and where do we go? Practical directions for faking research. In R. L. Griffith \& M. H. Peterson (Eds.), A closer examination of applicant faking behavior (pp. 357-371). Greenwich, CT: Information Age.

Savalei, V., \& Rhemtulla, M. (2013). The performance of robust test statistics with categorical data. British Journal of Mathematical and Statistical Psychology, 66 (2), 201-223. doi: 10.1111/j.2044-8317.2012.02049.x

Schaarschmidt, U. (2005). Halbtagsjobber? Psychische Gesundheit im Lehrerberuf. Analyse eines veränderungsbedürftigen Zustandes /Working only half of the day? Psychological well-being in the teacher profession. An analyses of a state needing change] (2. Auflage ed.). Weinheim/Basel: Beltz.

Schaarschmidt, U., \& Fischer, A. W. (2013). Inventar zur Persoenlichkeitsdiagnostik in Situationen [Inventory for Personality Assessment in Situations] (No. Version 21 - Revision 2). Mödling: Schuhfried.

Schaarschmidt, U., \& Kieschke, U. (2007). Gerüstet für den Schulalltag. Psychologische Unterstützungsangebote für Lehrerinnen und Lehrer [Being prepared for everyday school life. Psychological support measures for teachers]. Weinheim/Basel: Beltz.

Snell, A. F., Sydell, E. J., \& Lueke, S. B. (1999). Towards a theory of applicant faking: Integrating studies of deception. Human Resource Management Review, 9(2), 219-242. doi: 10.1016/S1053-4822(99)00019-4

Tett, R. P., \& Simonet, D. V. (2011). Faking in Personality Assessment: A "Multisaturation" Perspective on Faking as Performance. Human Performance, 24(4), 302-321. doi: 10.1080/08959285.2011.597472

Trapmann, S., Hell, B., Weigand, S., \& Schuler, H. (2007). Die Validität von Schulnoten zur Vorhersage des Studienerfolgs - Eine Metaanalyse. Zeitschrift fur Padagogische Psychologie, 21(1), 11-27. doi: 10.1024/ 1010-0652.21.1.11

Vandenberg, R. J., \& Lance, C. E. (2000). A review and synthesis of the measurement invariance literature: Suggestions, practices, and recommendations for organizational research. Organizational Research Methods, 3(1), 4-70. doi: 10.1177/109442810031002

Viswesvaran, C., \& Ones, D. S. (1999). Meta-analyses of fakability estimates: Implications for personality measurement. Educational and Psychological Measurement, 59(2), 197-210. doi: 10.1177/00131649921969802

Winkelspecht, C., Lewis, P., \& Thomas, A. (2006). Potential effects of 
faking on the NEO-PI-R: Willingness and ability to fake changes who gets hired in simulated selection decisions. Journal of Business and Psychology, 21 (2), 243-259. doi: 10.1007/s10869-006-9027-4

Zickar, M. J., \& Robie, C. (1999). Modeling faking good on personality items: An item-level analysis. Journal of Applied Psychology, 84 (4), 551-563. doi: 10.1037/0021-9010.84.4.551

Ziegler, M. (2011). Applicant Faking: A Look Into the Black Box. TIP: The Industrial-Organizational Psychologist, 49(1), 29-36. 